\title{
The Fixed Point Index for Local Condensing Maps.
}

\author{
by Roger D. Nussbaum (New Jersey, U.S.A.) (*)
}

\begin{abstract}
Summary. - We define below a fixed point index for local condensing maps $f$ defined on open subset of "nice. metric ANR's. We prove that all the properties of classical fixed point index for continuous maps defined in compact polyhedra have appropriate generalizations. If our map is compact (a special case of a condensing map) and defined on an open subset of a Banach space, we prove that our fixed point index agrees with LeraySchauder degree.
\end{abstract}

\section{Introduction.}

Let $X$ be a Banach space, $G$ an open subset of $X$, and $f: \bar{A} \rightarrow X$ a compact map. For some $a \in X$ suppose that $(I-f)^{-1}(a)$ is a compact subset of $G$. Under these assumptions Leray and SchaUder [24], using results of Brouwer [4], defined the topological degree of $I-f$ on $G$ over the point $a$, $\operatorname{deg}(I-f, G, a)$. The Leray-SchadDer degree has proved to be one of the most powerful and subtle tools for the study of fixed points of $f$ or of the properties of the map $I-f, f$ compact. It has had significant applications to partial differential equations, ordinary differential equations, and integral equations.

It was realized later that the so-called topological fixed point index (discussed in varying degrees of generality in [1], [6], [14], [22], and [37]) could be used to obtain the LERAY-SchaUDER degree. If $C$ is a «nice» compact, HAUSDORFF space (for instance, a compact polyhedron or a finite union of compact convex sets in a Banach space), 0 is an open subset of $C$, and $g: 0 \rightarrow C$ is a continuous map such that $\{x \in 0: g(x)=x\}$ is compact or empty, then the topological fixed point index of $g, i_{C}(g, 0)$, is defined. This integer reduces to the Lefschetz number when $C=0$. If $f$ and $G$ are as above and $G$ is bounded, then if we set $C=$ convex closure of $(f(x)+a$ : $: x \in G\}, 0=G \cap C$, and $g(x)=f(x)+a$ for $x \in 0, \operatorname{deg}(I-f, G, a)=i_{c}(g, 0)$.

In recent years it has become clear that it is desirable to extend degree theory to much more general maps than compact displacements of the identity. Extensions along this line have been given by BrowDEn and Nussbaum in [10]; an exposition of this work is given by BrowDer in [9].

(*) Entrata in Redazione il 12 ottobre 1970. 
In this paper we shall give a different generalization of the LERAY-SOHAUDER degree. We shall consider maps of the form $I-f$, where $f$ is a $k k$-setcontraction », $k<1$, and define a degree theory for them. The simplest nontrivial example of a $k$-set-contraction, $k<1$, is a map of the form $U+C, U$ a strict contraction defined on some subset of a Banach space, and $C$ a compact map. Actually, we shall consider maps $f: G \rightarrow X$, where $f$ is a $k$-set-contraction, $k<1$, and $G$ is an open subset of a «nice » metric ANR $X$; and we shall define a fixed point index $i_{X}(f, G)$. We shall prove that our definition agrees with the classical one when $X$ is a compact polyhedron, and we shall show that all the properties of the usual fixed point index - the additivity, homotopy, aud normalization properties extend in the appropriate way to our context. Our fixed point index will give as a special case a degree theory for maps of the form $I-f$.

Because of problems of length we cannot give applications of our fixed point index in this paper though some of the standard applications will be clear to anyone familiar with LERAX-SoHAUDER degree. In [32] we apply our fixed point index in its full generality in order to obtain asympotic fixed point theorens for $k$-set-contractions. These results generalize theorems of Browder in [8]. In [33] we specialize to the case of degree theory. We prove an invariance of domain theorem for our maps, we investigate questions of $A$-properness and obtain results along the lines of Browder and Petry. SHXN in [11], and we prove that our degree theory agrees with that defined by Browder and Nussbaum [10] where they coincide. Some of these results have been summarized in [28]. In a future paper we shall give applications to an existence theorem for a hyperbolic partial differential equation and local existence theorems for equations of evolution and functional differential equations. Preliminary results along this line are given in [30] and [31].

Some remarks about the organization of this paper: Section $A$ gives definitions, notations, and basic theorems from the literature, though some of the results are new. In Section $B$ we study geometrical properties of finite unions of convex sets in a vector space; our results can be viewed as a generalization of DUGUNDJ's theorem [15] that a closed, convex subset $C$ of a Banach space $X$ is a retract of $X$. Section $C$ presents extensions of the classical fixed point index which are necessary for the later work. In Section $D$ the fixed point index is defined for a class of maps which includes $k$-set-contractions, $k<1$. This class, though somewhat clumsy to work with, is actually quite useful in applications and provides greater flexibility than the $k$-sel-contractions. See [30], for instance. In Section $E$ we apply our previous work to local strict-set-contractions and spend considerable space in relating LERAX's generalized LEFsOHETz number [23] to our fixed point index. In the final section we uso a sort of limit argument to define a fixed point index for certain 1-set-contractions and especially for the "condensing 
maps » considered in [3] and [36] (see also [38] and the remark at the end of this paper).

The reader who is only intersted in degree theory can avoid considerable effort. For degree theory the results of Section $B$ are unnecessary; one only needs DugundJi's theorem [15]. The technical difficulties encountered in Section $F$ in the general case completely vanish for this special case. Finally, for degree theory one can assume throughout that the metric ANR's considered are closed, convex subsets of Banach spaces.

Aside from the material on condensing maps, the theorems given here were proved in the author's Ph.D. dissertation (University of Chicago, 1969) and were summarized in [28].

\section{A. - The measure of noncompactness and $k$-set-contractions.}

Let us begin with some basic notions. If $(A, \rho)$ is a bounded metric space, we define $\gamma(A)$, the measure of noncompactness of $A$, to be inf $\{d>0 \mid A$ can be covered by a finite number of sets of diameter less than or equal to $d\}$. The idea of measure of noncompactness is due to KuRATowskI [20]. If $(X, \rho)$ is a metric space and $A$ is a bounded subset of $A, A$ inherits a metric from $X$, and we can speak of $\gamma(A)$. Alternatively in this case, we can define $\gamma^{\prime}(A)=\inf |d>0| A$ can be covered by a finite number of sets in $X$ (sets not necessarily contained in $A$ ) of diameter less than or equal to $d$ ) and it is clear that $\gamma^{\prime}(A)=\gamma(A)$. In practice, when we speak of measure of noncompactness, it will almost invariably be the measure of noncompactness of a bounded subset $A$ of a metric space $(X, \rho)$.

In this generality little can be said about the measure of noncompactness. Our first proposition lists the essentials. We leave the proof to the reader.

Proposimion.1. - Let $(X, \rho)$ be a metric space and $A$ and $B$ bounded subsets of $X$. We write $N_{r}(A)=\{x \in X: \rho(x, A)<r\}$. Then we have a) if $A \subset B, \gamma(A) \leq \gamma(B)$ b) $\gamma\left(N_{r}(A)\right) \leq \gamma(A)+2 r$ c) if $c 1(A)$ denotes the closure of $A, \gamma(c 1(A))=\gamma(A)$ d) $\gamma(A \cup B)=\max (\gamma(A), \gamma(B)\}$.

If we assume that $(X, \rho)$ is a complete metric space, we can say considerably more. Recall that in a complete metric space $(X, \rho)$ a subset $\dot{A}$ has compact closure iff it is totally bounded, and clearly $A$ is totally bounded iff $\gamma(A)=0$. We also know that a decreasing sequence of compact, nonempty spaces has nonempty intersection. The following theorem, due to KuRATowskI [20], generalizes the latter result. We include a proof for completeness.

Proposition 2. (Kuratowski) - Let $(X, \rho)$ be a complete metric space and let $A_{1} \supset A_{2} \supset \ldots$ be a decreasing sequence of nonempty, closed subsets of $X$. 
Assume that $\gamma\left(A_{n}\right)$ converges to 0 . Then if we write $A_{\infty}=\bigcap_{n \geq 1} A_{n}, A_{\infty}$ is a nonempty compact set and $A_{n}$ appronches $A_{\infty}$ in the HAUSDORFF metric.

Proof. - Since $\gamma\left(A_{\infty}\right) \leq \gamma\left(A_{n}\right)$ for all $n$, it is clear that $\gamma\left(A_{\infty}\right)=0$, and since $A_{\infty}$ is an intersection of closed sets, it is closed. It follows that $A_{\infty}$ is compact. We have to show $A_{\infty}$ is nonempty.

Let us write $\gamma\left(A_{n}\right)=d_{n}$ and select a sequence of positive numbers $\varepsilon_{n}$ such that $\lim _{n \rightarrow \infty} \varepsilon_{n}=0$. By assumption we can write $A_{n}=\bigcup_{j=1}^{k(n)} S_{j, n}$, where diam $\left(S_{j, n}\right) \leq d_{n}+\varepsilon_{n}, 1 \leq j \leq k(n)$. We know that for some $j, 1 \leq j \leq k(1), S_{j, 1} \cap A_{n}$ is nonempty for all $n$. Otherwise, we would have $A_{n}=A_{1} \cap A_{n}=\left(\bigcup_{j=1}^{k(1)} S_{j, 1}\right) \cap A_{n}$ empty for $n$ large enough, a contradiction. Let us denote by $T_{1}$ the $S_{j, 1}$ such that $S_{j, 1} \cap A_{n} \neq \varnothing$ for all $n$.

We now proceed by induction. Assume we have found $T_{1}, T_{2}, \ldots, T_{m}$ such that $T_{i}=S_{j, i}$ for some $j, 1 \leq j \leq k(i)$, and such that $\left(\bigcap_{i=1}^{m} T_{i}\right) \cap A_{n}$ is is nonempty for all $n$. Let us write $T=\bigcap_{i=1}^{m} T_{i}$. We want to find $T_{m+1}=S_{j, m+1}$ for some $j, 1 \leq j \leq k(m+1)$ such that $T \cap T_{m+1} \cap A_{n}$ is nonempty for all $n$. If for each $j, 1 \leq j \leq k(m+1), T \cap S_{j, m+1} \cap A_{n}$ is empty for some $n$, then for $n$ large enough, $T \cap A_{n}=T \cap A_{m+1} \cap A_{n}=\bigcup_{j=1}^{k(m+1)} T \cap S_{j, m+1} \cap A_{n}$ is empty, a contradiction. Thus for some $j, 1 \leq j \leq k(m+1), T \cap S_{j, m+1} \cap A_{n}$ is non. empty for all $n$. We define $T_{m+1}=S_{j, m+1}$, and the induction is complete.

By our inductive process we know that $\bigcap^{n} T_{j}$ is nonempty and contained in $A_{n}$. Select $x_{n} \in \bigcap_{j=1}^{n} T_{j}$. Since $\operatorname{diam}\left(\bigcap_{j=1}^{m} T_{j}\right)^{j=m} \leq \operatorname{diam}\left(T_{m}\right) \leq d_{m}+\varepsilon_{m}$, we see that for $j$ and $k \geq m, d\left(x_{j}, x_{k}\right) \leq d_{m}+\varepsilon_{m}$. Thus $\left\{x_{j}\right\}$ is a CAUCHX sequenee and has a limit $x$. Since $x_{j} \in A_{m}$ for $j \geq m$, and since $A_{m}$ is closed, $x \in A_{m}$. It follows that $x \in \bigcap_{m \geq 1} A_{m}=A_{\infty}$.

It remains to show that $A_{n}$ approaches $A_{\infty}$ in the HAdSDORFF metric. Suppose not. Then for some $r>0$, we can find a subsequence $A_{n_{i}}$ such that $B_{i} \equiv A_{n_{i}} \cap N_{r}\left(A_{\infty}\right)^{\prime}$ is nonempty, where $N_{r}\left(A_{\infty}\right)^{\prime}$ denotes the complement of $N_{r}\left(A_{\infty}\right)$. Since $B_{i}$ is a decreasing sequence of nonempty closed sets with $\lim \gamma\left(B_{i}\right)=0$, what we have already proved shows $\underset{i \geq 1}{\cap} B_{i}$ is nonempty. But this is a contradiction, since $\cap_{i \geq 1} B_{i} \subset A_{\infty}$ and $\underset{i>1}{\cap} B_{i} \subset N_{r}\left(A_{\infty}\right)^{\prime} . \quad$ Q.E.D.

If we specialize still further and assume that $X$ is a BANACH space, we obtain results which will be crucial in our further work. The next two propositions are due to DARBo and can be found in his article [12]. The proofs are not difficult. 
Proposition 3. (Darbo). - Let $X$ be a Banaci space and suppose $A$ and $B$ are bounded subsets of $X$. If we denote $\{a+b \mid a \in A, b \in B\}$ by $A+B$, then $\gamma(A+B) \leq \gamma(A)+\gamma(B)$.

Proposimion 4. (Danbo). - Let $X$ be a Banadr space and $A$ a bounded subset of $X$. If we denote the convex closure of $A$ by $\operatorname{cocl}(A)$ (we may also write $\overline{c o}(A)$ for the convex closure of $A), \gamma(\operatorname{cocl}(A))=\gamma(A)$.

In general, given a subset $A$ of a BaNach space $X$, there is no easy algorithm to determine $\gamma(A)$. However, if $A$ is a ball in an infinite dimensional BANAOH space, we can describe $\gamma(A)$.

M. FurI and A. VIGNoli have also obtained this result [40], but we believe it was first proved in our dissertion [31].

Proposition 5. - Let $X$ be an infinite dimensional Banach space, let $B=\{x \mid\|x\| \leq 1\}$ and $S=\{x \mid\|x\|=1\}$. Then $\gamma(B)=\gamma(S)=2$.

Proof. - Since $B=\operatorname{cocl}(S)$, Proposition 4 implies that $\gamma(B)=\gamma(S)$, and since $\operatorname{diam}(S)=2$, it is certainly true that $\gamma(S) \leq 2$. To see that $\gamma(S)=2$, we proceed by contradiction. If $\gamma(S)<2$, we can write $S=\bigcup_{j=1}^{n} T_{j}$, where diam $\left(T_{j}\right)<2$, and by taking $\mathrm{cl}\left(T_{j}\right)$ instead of $T_{j}$ if necessary, we can assume $T_{j}$ is closed. Let $F$ be an $n$ dimensional subspace of $X$ and consider $S \cap F=\bigcup_{j=1}^{n}\left(T_{j} \cap F\right)$. By the LUSTERnIK-SchniRELMAN-Bonsuk theorem [17, p. 50], if the unit sphere (with respect to any norm) in an $n$-dimensional vector space $V$ is covered by $n$ closed sets, then at least one of the sets contains a pair of antipodal points, i.e., points $x$ and $-x$ for some $x$ on the unit sphere. In our case this means some $T_{j} \cap F$ contains a pair of antipodal points, so for this $T_{j} \cap F, 2 \leq \operatorname{diam}\left(T_{j} \cap F\right) \leq \operatorname{diam}\left(T_{j}\right)$, a contradiction.

Q.E.D.

We should remark that the LUSTERNIK-SOHNIRELMAN-BoRsUK theorem is proved in [17] for a particular norm on $R^{n}$, but since all norms on $R^{n}$ are equivalent it is easy to see that the unit spheres with respect to different norms are homeomorphic with a homeomorphism which takes antipodal po. ints to antipodal points. This shows the theorem true for any norm on $R^{n}$. For an arbitrary $n$ dimensional vector space $V$, we just select an isomorphism $I: V \rightarrow R^{n}$ and take a norm on $R^{n}$ such that the isomorphism is an isometry. The theorem for $V$ follows.

Closely associated with the notion of measure of noncompactness is the concept of «k-set-contractions 》 (also due to KuRatowski, [20]). Let $\left(X_{1}, d_{1}\right)$ and $\left(X_{2}, d_{2}\right)$ be metric spaces and suppose $f: X_{1} \rightarrow X_{2}$ is a continuous map. We say that $f$ is a $k$-set-contraction if given any bounded set $A$ in $X_{1}, f(A)$ 
1s bounded and $\gamma_{2}(f(A)) \leq k_{\gamma} \gamma_{1}(A)$. Of course $\gamma_{i}$ denotes the measure of noncompactness in $X_{i}, i=1$, 2. If $f$ is a $k$-set-contraction, we define $\gamma(f)=$ $=\inf \{k \geq 0 \mid f$ is a $k$-set-contraction $\}$. Given $f: X_{1} \rightarrow X_{2}$, we shall say that $f$ is a local strict-set-contraction if for all $x \in X_{1}$ there exists a neigborhood $N_{x}$ of $x$ such that $f \mid N_{x}$ is a $k_{x}$-set-contraction, $k_{x}<1$.

We shall also need a slight generalization of $k$-set-contractions, $l<1$, essentially dne to B. N. SadovskII [36] (SADovskr actually used a different measure of noncompactness). Given a continuons map $f: X_{1} \rightarrow X_{2}$ we say that $f$ is a condensing map if for every bounded set $A$ in $X_{1}$ such that $\gamma_{1}(A) \neq 0$, $\gamma_{2}(f(A))<\gamma_{1}(A)$. We say that $f$ is a local condensing map if every $x \in X_{1}$, has a neighborhood $N_{x}$ such that $f \mid N_{x}$ is a condensing map.

Of course every $k$-set-contraction with $k<1$ is a condensing map, but the converse is not true. To see this, let $\rho:[0,1] \rightarrow \mathbb{R}$ be a strictly decreasing, nonnegative function such that $\rho(0)=1$. Let $B$ denote the unit ball about 0 in an infinite dimensional BANACH space $X$ and consider the map $f: B \rightarrow B$ given by $f(x)=0\|x\| x$. We claim that $f$ is a condensing map, but that $f$ is not a $k$-set-contraction for any $k<1$. To see that $f$ is not a $k$-set-contraction for $k<1$, consider $f\left(V_{r}(0)\right.$ for $0<r \leq 1$, where $V_{r}(0)$ is the closed ball of radius $r$. It is easy to see that $f\left(V_{r}(0)\right) \supset V_{f(r) r}(0)$. By Proposition 5, $\gamma\left(V_{\varphi(r) r}(0)\right)=2 \rho(r) r$ and since $\gamma\left(V_{r}(0)\right)=2 r$, it follows that at best $f$ is a $\rho(r)$-set-contraction. Since $\rho(r) \rightarrow 1$ as $r \rightarrow 0, f$ cannot be a $k$-set-contraction for any $k<1$. On the other hand, $f$ is a $1-$ set-contraction, for if $A$ is any subset of $B, f(A) \subset \overline{c o}\{A \cup\{0\}\}$ and thus $\gamma f(A) \leq \gamma(A \cup\{0\})=\gamma(A)$. However, we can say more than this. Suppose $A \subset B$ and $\gamma(A)=d>0$. Select $r<d / 2$, define $A_{1}=A \cap V_{r}(0), A_{2}=A \cap V_{r}^{\prime}(0)\left(V_{r}^{\prime}(0)=\right.$ complement of $\left.V_{r}(0)\right)$, and consider $f(A)=f\left(A_{1}\right) \cup f\left(A_{2}\right)$. Since $f$ is a 1-set-contraction, $\left.\gamma f\left(A_{1}\right)\right) \leq 2 r<d=\gamma(A)$. Since $\rho$ is strictly decreasing and $\|x\| \geq r$ for $x \in A_{2}, f\left(A_{2}\right) \subset\{$ sa $\mid 0 \leq s \leq \rho(r)$, $\left.a \in A_{2}\right\} \subset \overline{\operatorname{co}}\{\rho(r) A \cup\{0\}\}$ and $\gamma\left(f\left(A_{2}\right)\right) \leq \rho(r) \gamma(A)<\gamma(A)$. It follows that $\gamma(f(A))=\max \left(\gamma\left(f\left(A_{1}\right)\right), \gamma\left(f\left(A_{2}\right)\right)\right\}<\gamma(A)$, so that $f$ is a condensing map. The same proof also shows that $f$ is not a local strict-set-contraction.

We now wish to state the elementary properties of $k$-set-contractions and give some examples which indicate their usefulness.

Proposition 6. (Darbo) a). - Let $\left(X_{i}, d_{i}\right), i=1,2,3$ be metrie spaces. Assume that $f: X_{1} \rightarrow X_{2}$ is a $k_{1}$-set-contraction and $g: X_{2} \rightarrow X_{3}$ is a $k_{2}$-setcontraction. Then $g f$ is a $k_{1} k_{2}$-set-contraction. b) Let $(X, d)$ be a metric space and $Y$ a BANACH space. Assume that $f: X \rightarrow Y$ is a $k_{1}$-set-contraction and $g: X \rightarrow Y$ is a $k_{2}$-set-contraction. Then $f+g$ is a $\left(k_{1}+k_{2}\right)$-contraction.

Proor. - a) Let $A$ be a bounded set in $X_{1}$. Then $f(A)$ is a bounded set in $X_{2}$ and $\gamma_{2}(f(A)) \leq k_{1} \gamma_{1}(A)$. Since $g$ is a $k_{2}$-set-contraction, $\gamma_{3}(g(f(A))) \leq k_{2} \gamma_{2}$ $(f(A)) \leq k_{1} k_{2} \gamma_{1}(A)$, i.e., $g f$ is a $k_{1} k_{2}$-set-contoaction. 
b) Let $A$ be a bounded set in $X$. Then $(f+g)(A) \subset f(A)+g(A)$, so by Proposition $3, \quad \gamma((f+g)(A)) \leq \gamma(f(A)+g(A)) \leq \gamma(f(A))+\gamma(g(A)) \leq k_{1} \gamma(A)+$ $+k_{2} \gamma(A)=\left(k_{1}+k_{2}\right) \gamma(A)$, so $f+g$ is a $\left(k_{1}+k_{2}\right)$-set-contraction. Q.E.D.

Notice that in the above we used $\gamma$ to refer to the measure of noncom. pactness in $X$ and in $Y$, even though they are different. We shall occasionally do this if it seems no confusion will result.

Let $\left(X_{1}, d_{1}\right)$ and $\left(X_{2}, d_{2}\right)$ be metric spaces and $f: X_{1} \rightarrow X_{2}$ a continuous map. We shall say that $f$ is compact on bounded sets or, occasionally, simply compact, if given a bounded set $A \subset X_{1}, f(A)$ has compact closure. We shall say that $f$ is a $k$-contraction if $d_{2}(f(x), f(y)) \leq k d_{1}(x, y)$ for every $x, y \in X_{1}$. We emphasize that $f$ being a $k$-contraction is much less general than its being a $k$-set-contraction.

Proposirton 7. (DARbo). - Let $\left(X_{1}, d_{1}\right)$ and $\left(X_{2}, d_{2}\right)$ be metric spaces and $f: X_{1} \rightarrow X_{2}$ a continuous map. a) If $f$ is a $k$-contraction, then $f$ is a $k$-setcontraction. b) If $f$ is compact on bounded sets, then $f$ is a 0 -set-contraction. Conversely, if $X_{2}$ is complete and $f$ is a 0 -set-contraction, then $f$ is compact on bounded sets.

Proof. - a) Let $A$ be a bounded set in $X_{1}$ and suppose $\gamma_{1}(A)=d$. Then given $\varepsilon>0$, we can write $A=\bigcup_{j=1}^{m} S_{j}$, diam $\left(S_{j}\right) \leq d+\varepsilon$. Thus $f(A)=\bigcup_{j=1}^{m} f\left(S_{j}\right)$, and since $f$ is a $k$-contraction, diam $\left(f\left(S_{j}\right)\right) \leq k(d+\varepsilon)$. Since $\varepsilon$ is arbitrary, $\gamma_{2}(f(A)) \leqslant k d$ and $f$ is a $k$-set-contraction.

b) Let $A$ be a bounded set in $X_{1}$. Since we are assuming $f$ is com. pact on bounded sets, $c l f(A)$ is compact and hence totally bounded. Thus $\gamma_{2}($ clf $(A))=\gamma_{2}(f(A))=0$, so $f$ is a 0 -set-contraction.

Conversely, if $X_{2}$ is complete and $f$ is a 0 -set-contraction, then for any bounded $A, \gamma_{2}(\operatorname{cl} f(A))=\gamma_{2}(f(A))=0$. This means clf $(A)$ is totally bounded, and since $X_{2}$ is complete, clf $(A)$ is compact, i.e., $f$ is compact on bounded sets.

Q.E.D.

Using Proposition 6 and 7 we can construct examples of $k$-set-contractions. For instance, let $G$ be a subset of a Barach space $X, U: G \rightarrow X$ a $k$-contraction and $C: G \rightarrow X$ compact on bounded sets. Then $U+C$ is a $k$-set-contraction.

In our next proposition we want to generalize the above example. Let $\left(X_{1}, d_{1}\right)$ and $\left(X_{2}, d_{2}\right)$ be metric spaces. Let $V: X_{1} \times X_{1} \rightarrow X_{2}$ be a continuous map. Assume that for $y \in X_{1}, V(\cdot, y): X_{1} \rightarrow X_{2}$ is a k-contraction, $k$ independent of $y$. Let $A$ be any bounded subset of $X_{1}$ and denote by $C\left(A, X_{2}\right)$ the metric space of bounded, continuous functions from $A$ to $X_{2}$. 
If $f, g \in C\left(A, X_{2}\right), d(f, g)$ is, by definition, $\sup _{x \in A} d_{2}(f(x), g(x))$. Assume that for $y \in A$, the map $y \rightarrow V(\cdot, y)$ form $A$ to $C\left(A, X_{2}\right)$ is compact on bounded sets.

Propositron 8, - Let $V: X_{1} \times X_{1} \rightarrow X_{2}$ be as above and let $f(x)=V(x, x)$. Then $f$ is a $k$-set-contraction.

Proof. - Let $A$ be a bounded subset of $X_{1}$. Since the map $y \rightarrow V(\cdot, y)$ is compact from $A$ to $C\left(A, X_{2}\right)$, we can select $y_{1}, \ldots, y_{n} \in A$, such that for $y \in A$, there exists $i$ such that $\sup _{x \in A} d_{2}\left(V(x, y), V\left(x, y_{i}\right)\right)<\varepsilon$. (This is just the translation of the fact that $\{V(\cdot, y)\} \subset C\left(A, X_{2}\right)$ is totally bounded in $\left.O\left(A, X_{2}\right)\right)$. If we write $V(\cdot, y)=V_{y}$, this means that $f(A) \subset V(A \times A) \subset \bigcup_{i=1}^{n} N_{\varepsilon}\left(V_{y_{i}}(A)\right)$. It follows that $\gamma_{2}(f(A)) \leq \max _{1 \leq i \leq n}\left\{\gamma_{2}\left(V_{y_{i}}(A)\right)+2 \varepsilon\right\}$. But since $V_{y_{i}}$ is a $k$-contraction, $\gamma_{2}\left(V_{y_{i}}(A)\right) \leq k \gamma_{1}(A)$, so $\gamma_{2}(f(A)) \leq k \gamma_{1}(A)+2 \varepsilon$, whence (since $\varepsilon$ can be taken as small as desired) $f$ is a $k$-set-contraction.

Q.E.D.

Mappings like $V$ are considered in [10], and we also encounter similar maps in applications.

Our next proposition indicates another way in which $k$-set-contractions may arise.

Proposimion 9. - Let $(X, d)$ be a metric space and $\left\{0_{i} \mid 1 \leq i \leq n\right\}$ be a finite open covering. Let $Y$ be a $B_{A N A C H}$ space and $f_{i}: 0_{i} \rightarrow Y$ a $k$-set-contraction, $1 \leq i \leq n$. Suppose that $\left\{\lambda_{i}\right\}$ is a partition of unit subordinate to the open covering $\left\{0_{i}\right\}$. Then the map $g(x)=\sum_{i=1}^{n} \lambda_{i}(x) f_{i}(x)$ is a $k$-set-contraction.

Proof. - Let $A$ be a bounded subset of $X$. Then since $\sum_{i=1}^{n} \lambda_{i}(x)=1$ for every $x \in A$, it is clear that $g(A) \subset$ co $\left[\bigcup_{i=1}^{n} f_{i}\left(A \cap 0_{i}\right)\right]$. It follows that $\gamma(g(A)) \leq$ $\leq \gamma\left(\operatorname{co}\left(\bigcup_{i=1}^{n} f_{i}\left(A \cap 0_{i}\right)\right)\right)=\gamma\left(\bigcup_{i=1}^{n} f_{i}\left(A \cap 0_{i}\right)\right)=\max _{1 \leq i \leq n} \gamma\left(f_{i}\left(A \cap 0_{i}\right)\right) \leq k \gamma(A)$. Q.E.D.

Corollarx 1. - Let $B=\{x:\|x\| \leq 1\}$ in a Banach space $X$ and let $R: X \rightarrow B$ be the radial projection, i.e. $R(x)=\frac{x}{\|x\|}$ for $\|x\| \geq 1$ and $R(x)=x$ for $\|x\| \leq 1$.
Then $R$ is a 1-set-contraction.

Proof. - Let $f_{1}(x)=x, f_{2}(x)=0, \lambda_{1}(x)=\frac{1}{\|x\|}$ for $\|x\| \geq 1$ and $\lambda_{1}(x)=1$ for $\|x\| \leq 1$, and $\lambda_{2}(x)=1-\lambda_{1}(x)$. Then $R(x)=\lambda_{1}(x) f_{1}(x)+\lambda_{2}(x) f_{2}(x)$, so Proposition 9 implies the result.

Q.E.D.

Corollary 1 is interesting, for as de Figuremo and $\mathrm{K}_{\text {ArLovitz }}$ have shown [16], if dimension $(X) \geq 3, R$ is a 1 -contraction if and only if $X$ is a HrLbert space. 
The apparatus of measure of noncompactness and $k$-set-contractions can be used to establish fixed point theorems. The proof of the following proposition is due to $\mathrm{D}_{\mathrm{ARB}}$, but $\mathrm{D}_{\mathrm{ABBO}}$ stated the proposition in less generality.

Proposimion 10. - Let $C$ be a closed, bounded, convex set in a Banach space $X$. Let $f: C \rightarrow C$ be a continuous map. Let $C_{1}=\overrightarrow{c o} f(C)$ and $C_{n}=\overline{\mathrm{co}}$ $f\left(C_{n-1}\right)$ for $n>1$. Assume that $\gamma\left(C_{n}\right) \rightarrow 0$. Then $f$ has a fixed point.

Proof. - It is clear that $O_{n}$ is closed, bounded, convex, and nonempty and $C_{n} \supset C_{n+1}$ for $n \geq 1$. By Proposition $2 O_{\bar{\infty}}^{=} \cap O_{n \geq 1}$ is nonempty and compact, and $C_{\infty}$ is certainly convex. By our construction $f: C_{n} \rightarrow C_{n+1}$ so that $f: C_{\infty} \rightarrow C_{\infty}$. It follows by Schauder's fixed point theorem that $f$ has a fixed point.

Q.E.D.

Corollary 2. - (Darbo, [12]). Let $C$ be a closed, bounded, convex set and $f: C \rightarrow C$ a $k$-set-contraction, $k<1$. Then $f$ has a fixed point.

Proof. - It suffices to show $\gamma\left(C_{n}\right) \rightarrow 0$. But $\gamma\left(C_{1}\right)=\gamma(\overline{\mathrm{co}} f(C))=\gamma(f(C)) \leq$ $\leq k \gamma(O)$, and generally $\gamma\left(C_{n}\right)=\gamma\left(\overrightarrow{c o} f\left(C_{n-1}\right)\right)=\gamma\left(f\left(C_{n-1}\right)\right) \leq k \gamma\left(C_{n-1}\right)$. This implies that $\gamma\left(O_{n}\right) \leq k^{n} \gamma(C)$.

Q.E.D.

Corollary 3. - Let $A$ be a bounded linear operator in a Banach space $X$ and assume lim sup $\gamma\left(A^{n}\right)^{1 / n}=k<1$. Let $C$ be a closed, bounded, convex set in $X$ and $B: C \rightarrow X$ a compact (not necessarily linear) map. Assume that if $f(x)=A(x)+B(x), f: C \mapsto C$. Then $f$ has a fixed point.

Proor. - In the notation of Proposition 10 it suffices to show $\gamma\left(C_{n}\right) \rightarrow 0$. To see this first notice that for $S \subset C, \gamma(f(\overline{\operatorname{co}} S))=\gamma(f(S))$. We have $\gamma(f(\overline{\mathrm{co}} S)) \geq \gamma(f(S))$, since $\overline{\mathrm{co}} S \supset S$. On the other hand $f(\overline{\mathrm{co}} S) \subset A(\overline{\mathrm{co}} S)+$ $+B(\overline{\mathrm{co}} S)$, so by Proposition $3, \gamma(f(\overline{\mathrm{co}} S)) \leq \gamma(A(\overline{\mathrm{co}} S))+\gamma(B(\overline{\mathrm{co}} S))=\operatorname{since} B$ is compact) $\gamma(A(\mathrm{co} S))$. Since $A$ is linear it follows that $A(\overline{c o} S)=\overline{\mathrm{co}} A(S)$, so $\gamma(A(\overline{\mathrm{co}} S)) \leq \gamma(\overrightarrow{\mathrm{co}} A(S))=\gamma(A(S))$. However, $A(x)=f(x)-B(x)$ so $A(S) \subset$ $\subset f(S)\}-B(S)=\{f(s)-B(s) \mid s \in S\}$. This implies that $\gamma(A(S)) \leq \gamma f(S))+$ $+\gamma(-B(S))=\gamma(f(S))$, and consequently $\gamma(f(\overline{\mathrm{co}} S)) \leq \gamma(f(S))$.

Notice that $f^{j}=A^{j}+B_{j}$, where $B_{j}: C \rightarrow X$ is a compact map and $A^{j}$ is, of course, a bounded linear map. Thus the above reasoning implies that $\gamma\left(f^{j}(\overline{\mathrm{co}} S)\right)=\gamma\left(f^{j}(S)\right)$ for $S \subset O$. Applying this relation repeatedly we see that $\gamma\left(C_{n}\right)=\gamma\left(f\left(C_{n-1}\right)\right)=\gamma\left(f\left(\overline{\text { co }} f\left(C_{n-1}\right)\right)=\gamma\left(f^{2}\left(C_{n-2}\right)\right)=\ldots=\gamma\left(f^{n}(C)\right)\right.$. However, $f^{n}=$ $=A^{n}+B_{n}$, where $B_{n}$ is a compact, so $\gamma\left(f^{n}(C)\right) \leq \gamma\left(A^{n}(C)\right)+\gamma\left(B_{n}(C)\right)=\gamma\left(A^{n}(C)\right)$. Since $\lim \sup \left(\gamma\left(A^{n}\right)\right)^{1 / n}=k<1$, for $n \geq n_{0}, \gamma\left(A^{n}(C)\right) \leq k^{n} \gamma(O) \rightarrow 0$. Q.E.D.

Corollary 3 was proved by $N_{A S H E D}$ and WONG [27] under the stronger assumptions that 1) $A x+B y \in C$ for all $x, y \in C$ and 2) $\lim _{n \rightarrow \infty}\left\|A^{n}\right\|^{1 / n}<1$. The 
number lim sup $\left(\gamma\left(A^{n}\right)\right)^{1 / n}$ is studied in [29] and in the author's dissertation. (University of Chicago, 1969) where it is proved that $\lim \sup \left(\gamma\left(A^{n}\right)\right)^{1 / n}=\sup$ $\{|\lambda|: \lambda \in \operatorname{ess}(A))\}$. Ess $(A)$ denotes the essential spectrum of $A$ as defined by Browder [7].

\section{B. - Geometrical properties of finite unions of convex sets.}

In this section we shall establish theorems concerning the geometric properties of finite unions of closed, convex sets in a locally convex topological vector space. These results will prove erucial for the theory of the fixed point index for $k$-set-contractions.

Let us begin by recalling some basic facts. We shall say that a topological space $X$ is an absolute neighborhood retract (abbreviated ANR) if given any metric space $M$, a closed subspace $A \subset M$, and a continuous map $f: A \rightarrow X$, there exists an open neighborhood $U$ of $A$ and a continuous map $F: U \rightarrow X$ such that $F(a)=f(a)$ for $a \in A ; X$ is called an absolute retract $(A R)$ if $F$ as above can be defined on all of $M$. A theorem of Dugundur [15] asserts that any convex subset of a locally convex topological space is an $A R$. A classical result says that if $X_{1}$ and $X_{2}$ are subspaces of a topological space $Y$ and $X_{1}, X_{2}$, and $X_{1} \cap X_{2}$ are ANR's, then $X_{1} \cup X_{2}$ is an ANR. This implies immediately that if $C_{i}, 1 \leq i \leq m$, are convex subsets of some letvs $X$, then $C=U_{i=1}^{m} C_{i}$ is an ANR. It follows that if we assume $C_{i}, 1 \leq i \leq m$, is a closed. convex subset of a metrizable subset $Y$ of a letvs $X$, then there exists an open neighborhood $U$ in $Y$ of $C=U_{i=1}^{n} C_{i}$ and a retraction $R: U \rightarrow C$, simply because the identity map $i: C \rightarrow C$ can be extended as a map from an open neigborhood of $C$ to $C$ in this case. It is this fact which we shall use repeatedly.

With the aid of these results we can establish an essential lemma.

Lrmma 1. - Let $Y$ be a closed, metrizable subset of an letrs $X$. Let $C \subset Y$ be a finite union of $m$ closed, convex sets $C_{i}, C=U_{i=1}^{m} C_{i}$, and let $D \subset C$ be a finite union of closed, convex sets $D_{i} \subset C_{i}, D=U_{i=1}^{m} D_{i}$. Then there exists in $C$ an open neighborhood $V$ of $D$ and a retraction $s: V \rightarrow D$ such that $s(x) \in C_{i}$ if $x \in C_{i}$.

Proof. - First, let us establish some notation. We shall denote by $J, K$, $L, M$ subsets of $\{1,2, \ldots, m\}$ and by $|J|$ the number of elements of $J$. We shall write $C_{J}=n_{j \in J} C_{j}$ and $D_{J}=n_{j \in J} D_{j}$.

We begin with our map $s$ defined and equal to the identity $i$ on $D$, and we want to extend $s$. First, let us extend $s$ to a closed neighborhood $U_{K}$ in $C_{K}$ of $C_{K} \cap D,|K|=m$. (We allow the possibility that $C_{K}$ is empty, in which case $U_{K}$ is taken to be empty). Notice that $C_{K} \cap D$ is a finite union 
of closed, convex sets contained in a metrizable subset of an letvs, so that $C_{K} \cap D$ is an ANR (see Section $O$ ). Restricting our attention to $C_{K},|K|=m$, we thus see that there exists a closed neighborbood $U_{K}$ in $C_{K}$ of $C_{K} \cap D$ and a retraction $s_{K}: U_{K} \rightarrow C_{K} \cap D$. Let us write $s_{m}=i \cup s_{K}$ and $Z_{m}=C \cup U_{K}$, $|K|=m$. It is elear that $s_{m}$ is a retraction of $Z_{m}$ onto $D$ and that $s_{m}(x) \in C_{j}$ if $x \in C_{j} \cap Z_{m}$.

Now let us proceed inductively to find $s$ and $V$. Suppose we have found closed neighborhoods $U_{L}$ in $C_{L}$ of $C_{L} \cap D$ for all $L$ such that $|L|=p$, $m \geq p>1$, and suppose we also have a retraction $s_{p}: Z_{p}=D \cup \cup_{\mid L_{\mid}=p} U_{L} \rightarrow D$ such that $s_{p}: Z_{p} \cap C_{j} \rightarrow C_{j}$ for $1 \leq j \leq m$. Consider $C_{K},|K|=p-1$, and notice that by our assumptions $s_{p}: Z_{p} \cap C_{K} \rightarrow D \cap C_{K}$. Since $Z_{p} \cap C_{K}$ is a closed subset of the metric space $C_{K}$ and since $D \cap C_{K}$, a finite union of closed, convex, metrizable sets, is an ANR, there exists an extension of the map $s_{p}: Z_{p} \cap C_{K} \rightarrow D \cap C_{K}$ to an open neighborhood $U_{K}^{\prime}$ of $Z_{p} \cap C_{K}$ in $C_{K}$. Let us call this extended map $s_{K}$, so $s_{K}: U_{K}^{\prime} \rightarrow D \cap C_{K}$ is a retraction.

It is necessary to consider a smaller neighborhood than $U_{K}^{\prime}$. Notice that $U_{|L|=P} U_{L}$ is a neighborhood in $U_{\mid L=P} C_{L}$ of $D \cap\left(U_{|L|=p} C_{L}\right)$. Since $U_{|L|=p} C_{L}$ is closed in $C, \mathrm{C}_{K} \sim U_{|L|={ }_{p}} O_{L}$ is open in $C_{K}$. Thus it is not difficult to see that $W_{K} \equiv\left(C_{K} \sim U_{\mid L_{\mid}=p} C_{L}\right) \cup\left(U_{|L|=p}\left(U_{L} \cap C_{K}\right)\right)$ is a neighborhood in $C_{K}$ of $C_{K} \cap D$. This implies that $U_{K}^{\prime} \cap W_{K}$ is a neighborhood in $C_{K}$ of $C_{K} \cap D$. We take $U_{K}$ to be a closed neighborhood of $C_{K} \cap D$ in $C_{K}$ such that $U_{K} \subset U_{K}^{\prime} \cap W_{K}$, and we define $Z_{p-1}=D \cup U_{|K|=p-1} U_{K}$ and $s_{p-1}=s_{p} \cup\left(U_{|K|=p-1}\left(s_{K} \mid U_{K}\right)\right)$.

Our first claim is that this definition actually gives a well-defined re. traction of $Z_{p-1}$ onto $D$. To see this it suffices to show that if $|K|=\left|K^{\prime}\right|=p-1$, $K \neq K^{\prime}$, and $x \in U_{K} \cap U_{K^{*}}$, then $s_{K}(x)=s_{K^{\prime}}(x)$. But $U_{K} \cap U_{K^{\prime}} \subset C_{K^{\prime} \cup K^{\prime}}$ and $\left|K \cup K^{\prime}\right| \geq p$, so it follows, since $U_{K} \subset W_{K}$, that $x \in U_{J}$ for some $J$ with $|J|=p$. Since all the $s_{K}$ reduce to $s_{p}$ on $U_{J},|J|=p, s_{K}(x)=s_{p}(x)=s_{K^{\prime}}(x)$.

Next we have to show that if $x \in Z_{p-1} \cap C_{j}, s_{p-1}(x) \in C_{j}, 1 \leq j \leq m$. This condition is certainly satisfied if $x \in D$, so suppose that $x \in U_{K} \cap C_{j}$, $|K|=p-1$. If it is also true that $x \in U_{L}$ for some $L$ such that $|L|=p$, then by our construction $s_{p-1}(x)=s_{p}(x)$ and by inductive hypothesis $s_{p}(x) \in C_{j}$. If $x \in U_{K} \cap C_{j}$ but $x \notin U_{\mid L_{i}^{\prime}=p} U_{L}$ we know by the construation of $U_{K}$ that $x \in C_{K} \sim U_{|L|=p} C_{L}$. But we also know that $x \in U_{K} \cap O_{j} \subset C_{K \cup \mid j\}}$, so we must have that $j \in K$-otherwise $|K \cup\{j\}| \geq p$. It follows that $C_{K} \subset C_{j}$ in this case, and since wo constructructed $s_{p \rightarrow 1}$ so that $s_{p-1}: U_{K} \rightarrow C_{K} \cap D \subset C_{j}$, $s_{p-1}(x) \in C_{j}$.

This completes the inductive step. After earrying through the above construction $m$ times, we obtain $s_{m}=s$ and $Z_{m}$, which contains an open neighborhood $V$ of $D$.

Q.E.D.

Our next lemma is somewhat artificial, but it will prove crucial in esta. blishing our theorems. 
Lemma 2. - Let $Y$ be a closed, metrizable subset of an letvs $X$. Let $C \subset Y$ be a finite union of elosed, convex sets $C_{i}, C=U_{i=1}^{m} C_{i}$. For each subset $J \subset\{1,2, \ldots, m\}$, let $O_{J}$ denote an open neighborhood of $C_{J}=\bigcap_{j \in J} C_{j}$ if $C_{J}$ is nonempty and let $O_{J}$ denote the empty set if $C_{J}$ is empty. Then there exists an open covering $\left\{U_{J}\right\}$ of $C$ (indexed by subsets $\left.J \subset\{1,2, \ldots, m\}\right)$ which satisfies the following properties:

(1) $U_{J} \subset O_{J}$.

(2) If $|K| \geq|J|$ but $K \downarrow J, U_{K} \cap U_{J}$ is empty.

(3) If $j \notin K, \operatorname{el}\left(U_{k}\right) \cap C_{j}$ is empty.

Proof. - We shall construet $\left\{U_{K}\right\}$ inductively, starting with $|K|=m$, then considering $|K|=m-1$, and so on. For $|K|=m$, so that $K=\{1,2, \ldots, m\}$, we define $U_{K}=O_{K}$. Notice that $U_{|K| \geq m} U_{k} \supset U_{|K| \geq m} C_{K}$ and that $\left\{U_{K}|| K \mid \geq m\right\}$ trivially satisfies conditions (1)-(3).

Assume that we have constructed open sets $U_{L}$ in $C$ for $|L| \geq r, m \geq r>1$, such that $U_{|L| \geq r} U_{L} \supset U_{|L| \geq r} C_{L}$ and $\left\{U_{L}|| L \mid \geq r\right\}$ satisfies conditions (1)-(3) above. Select $K \subset\{1,2, \ldots, m\}$ with $|K|=r-1$. We wish to define $U_{K}$. Let $A_{K}=C_{K} \sim U_{|L| \geq r} U_{L}$ and notice that for $j \notin K, A_{K}$ and $C_{j}$ are disjoint closed sets. Thas there exists an open neighborhood $V_{K}$ of $A_{K}$ such that $V_{K} \subset O_{K}$ and $\mathrm{cl}\left(V_{K}\right) \cap O_{j}$ is empty for $j \notin K$. Consider all $L \subset\{1,2, \ldots, m\}$ such that $|L|>|K|=r-1$ but $L \nsupseteq K$, and for each such $L$, select $j \in K$ such that $j \notin L$. If $(L, j)$ is such a pair, we know by the inductive hypothesis that $\operatorname{cl}\left(U_{L}\right) \cap C_{j}$ is empty, so there exists an open neighborhood $W_{(L, j)}$ of $C_{j}$ such that $\operatorname{cl}\left(U_{L}\right) \cap W_{L, j}$ is empty. Since $C_{j} \supset C_{K} \supset A_{K}, W_{(L, j)}$ is an open neighborhood of $A_{K}$. Let us set $W_{K}=\bigcap_{(L, j)} W_{(L . j)}$, where the intersection is taken over all pairs $(L, j)$ such that $|L|>|K|, L \downarrow K$, and $j \in K$ but $j \notin L$. Finally, we note that for $K, K^{\prime} \subset\{1,2, \ldots, m\},|K|=\left|K^{\prime}\right|=r-1, K=K^{\prime}$, $A_{K} \cap A_{K^{\prime}}$ is empty, since $A_{K} \cap A_{K^{\prime}} \subset C_{K \cup K^{\prime}}$, and $\left|K \cup K^{\prime}\right| \geq r$. Thus it is not hard to see that we can find open neighborhood $Z_{K}$ of $A_{K}$ for $|K|=r-1$ such that for any two unequal subsets $K$ and $K^{\prime}$ with $|K|=\left|K^{\prime}\right|=r-1$, $Z_{K} \cap Z_{K^{\prime}}$ is empty. We define $U_{K}=\nabla_{K} \cap W_{K} \cap Z_{K}$, and we have to show that $\left\{U_{K}|| K \mid \geq r-1\right\}$ satisfies the inductive hypotheses.

Since $U_{K},|K|=r-1$, is an open noighborhood of $A_{K}$, we clearly have $U_{|K| \geq r-1} C_{K} \subset\left(U_{|K| \geq r} U_{K}\right) \cup\left(U_{|K|=r-1} A_{K}\right) \subset\left(U_{|K| \geq r-1} U_{K}\right)$. We selected $V_{K} \subset O_{K}$, so the first condition on the covering is satisfied. To check the second condi. tion, we must show that if $L \subset\{1,2, \ldots, m\},|L| \geq|K|=r-1$, and $L \supset K$, then $U_{L} \cap U_{K}$ is empty. If $|L|>|K|$, select $j \in K$, such that $j \notin L$. By construction we have $U_{L} \cap U_{K} \subset U_{L} \cap W_{(L, j)}$, which is empty. If $|L|=|K|$, we have $U_{L} \cap U_{K} \subset Z_{L} \cap Z_{K}$, which is empty. Finally, to check the third condition, we have to show that if $K \subset\{1,2, \ldots, m\},|K|=r-1$ and $j \notin K$, 
then $\operatorname{cl}\left(U_{K} \cap C_{j}\right.$ is empty. But $U_{K} \subset V_{K}$, so $\operatorname{cl}\left(U_{K}\right) \cap C_{j} \subset \operatorname{cl}\left(V_{K}\right) \cap C_{j}$, which is empty.

This completes the inductive step. After $m$ steps we obtain the desired covering.

We can now prove our main theorem.

Theorem 1. - Let $Y$ be a closed, metrizable subset of an letvs $X$. Let $C \subset Y$ be a finite union of $m$ closed, convex sets $C_{i}, C=\bigcup_{i=1}^{m} C_{i}$ and let $D=\bigcup \bigcup_{i=1}^{m} D_{i}$ be a finite union of $m$ closed, convex sets $D_{i}$ such that $D_{i} \subset C_{i}$. For every subset $J \subset(1,2, \ldots, m)$ assume that $D_{J} \equiv \bigcap_{j \in J} D_{j}$ is empty if and only if $C_{J} \equiv \bigcap_{j \in J} C_{j}$ is empty. Then there exists a retraction $R$ of $C$ onto $D$ such that $R(x) \in C_{i} \cap D$ if $x \in C_{i}, 1 \leq i \leq m$. (In particular, $D$ is a deformation retract of $C$ by the deformation retraction $H(x, t)=(1-t) R(x)+t x, 0 \leq t \leq 1)$. Furthermore, let $p$ be a continuous seminorm on $X$ and $\varepsilon$ a positive number. Then there exist open neighborhoods $\tilde{O}_{K}$ in $Y$ of $D_{K}$ for $K \subset(1,2, \ldots, m\}$ ( $\tilde{O}_{K}$ is empty if $D_{K}$ is empty) such that if $C_{K} \subset \tilde{O}_{K}$ for all $K, R$ can be choosen so that $p(R x-x)<\varepsilon$ for $x \in C$.

Proof. - For each nonempty $D_{K}$, let $r_{K}: Y \rightarrow D_{K}$ be a retraction of $Y$ onto $D_{K}$. Such retractions exist since $D_{K}$ is an $\mathrm{AR}[19]$. Let $\tilde{O}_{K}=\{x \in Y \mid$ $\left.p\left(\boldsymbol{r}_{K}(x)-x\right)<\varepsilon\right\}$ if $D_{K}$ is nonempty and $\tilde{O}_{K}$ be empty otherwise. If $C_{K} \subset \tilde{O}_{K}$ for all $K$, let $O_{K}=\tilde{O}_{K} \cap C$. Otherwise, let $O_{K}$ be any open neighborhood of $C_{K}$ in $C$ such that $O_{K}$ is empty if $C_{K}$ is empty. By Lemma 2 there exists an open covering $\left\{U_{K}\right\}$ of $C$ such that: (1) $U_{K} \subset O_{K}$; (2). If $|L| \geq|K|$ but $L \nsupseteq K$, $U_{L} \cap U_{K}$ is empty; (3). If $j \notin K, \operatorname{cl}\left(U_{L}\right) \cap C_{j}$ is empty. By Property 3 of the covering it is elear that for all $L \subset\{1,2, \ldots, m\}$ there exists an open neighborhood $W_{L}$ of el $\left(U_{L}\right)$ such that $W_{L} \cap C_{j}$ is empty for $j \notin L$. By Lemma 1 there exists an open neighborhood $V$ of $D$ in $C$ and a retraction $s: V \rightarrow D$ such that $s: V \cap C_{i} \rightarrow D \cap C_{i}$. Let $W=\{x \in V \mid \alpha) p(s(x)-x)<\varepsilon$ and b). If $x \in \operatorname{cl}\left(U_{L}\right)$ for any $L \subset\{1,2, \ldots, m\}$, then $\left.s(x) \in W_{L}\right\}$. Since $s(x)=x$ for $x \in D$, it is clear thet $D \subset W$; and it is not hard to see that $W$ is open. Thus $W$ is an open neighborhood of $D$. Let $W_{1}$ be a closed neighborhood of $D$ such that $W_{1} \subset W$ and denote by $W_{1}^{\prime}$ the complement of $W_{1}$ in $C$. Consider the open covering of $C$ by $\left(W, U_{K} \cap W_{1}^{\prime}\right\}$ and let $\left\{\theta, \theta_{K}\right\}$ denote a partition of unity subordinate to this covering, i.e., $\operatorname{supp}(\theta) \subset W, \operatorname{supp}\left(\theta_{K}\right) \subset U_{K} \cap W_{1}^{\prime}$ and $\theta(x)+\sum_{K} \theta_{K}(x)=1$ for $x \in C$. We define $R(x)=\theta(x) s(x)+\Sigma_{K} \theta_{K}(x) r_{K}(x)$; the summation, of course, is only over $K$ such that $D_{K}$ is nonempty and $\theta(x) s(x)$ is defined to be 0 for $x \notin V$.

We have to show that $R$ satisfies the claims of the theorem. First, let as prove that if $x \in C_{j}, R(x) \in C_{j}$. If $j \notin L \subset\{1,2, \ldots, m\}$, we know that $U_{L} \cap C_{j}$ is empty. Thus $\theta_{L}(x)=0$ unless $j \in L$, and for such $L, r_{L}(x) \in D_{L} \subset C_{j}$. If $x \in V$, so that $s(x)$ is defined, $s(x) \in C_{j}$. Thus $R(x)=\theta(x) s(x)+\sum_{K} \theta_{K}(s) s_{K}(x)$ is a convex combination of points in $C_{j}$ and hence lies in $C_{j}$. 
Next let us show that $R$ is actually a retraction of $C$ onto $D$. Since $R(x)=s(x)$ for $x \in W_{1}$, to show $R$ is a retraction it suffices to show $R(x) \in D$ for $x \in C$. Given $x \in C$, select $J$ such that $x \in U_{J}$ but $x \notin U_{L}$ for $|L|>|J|$. Furthermore, if $|L| \geq|J|$, we know that $U_{L} \cap U_{J}$ is empty unless $L \supset J$, so that $\theta_{L}(x)=0$ unless $L \supset J$. Now consider $R(x)=\theta(x) s(x)+\Sigma_{K} \theta_{K}(x) r_{K}(x)$. If $\theta(x)=1, R(x)=s(x) \in D$. If $\theta(x)<1, R(x)=\theta(x) s(x)+(1-\theta)) \sum_{K}\left(\theta_{K}(x) / 1-\theta(x) r_{K}(x)\right.$. Since $r_{K}(x) \in D_{K} \subset D_{J}$ for $K \supset J$ and since $\theta_{K}(x)=0$ unless $K \supset J, \sum_{K}\left(\theta_{K}(x) /\right.$ $1-\theta(x)) r_{K}(x)$ is a convex combination of points in $D_{J}$ and hence lies in $D_{J}$. If $\theta(x)=0$, we are done. If $\theta(x)>0$, we have $x \in W$, so that $s(x) \notin C_{k}$ for $k \notin J$ and consequently $s(x) \notin D_{k}$ for $k \notin J$. It follows that $s(x) \in D_{j}$ for some $j \in J$. Thus $R(x)$ is a convex combination of points in $D_{j}$ and lies in $D_{j} \subset D$.

Finally, let us suppose that $C_{K} \subset \tilde{O}_{K}$ for all $K$. Then by our construction $\theta_{K}(x)=0$ unless $p\left(r_{K}(x)-x\right)<\varepsilon$. Also by construction, $\theta(x)=0$ unless $p(s(x)-x)<\varepsilon$. It follows that $p(R(x)-x) \leq \theta(x) p(s(x)-x)+\Sigma_{K} \theta_{K}(x) p\left(r_{K}(x)-x\right)<\varepsilon$. Q.E.D.

Corollary 1. - Let $Y$ be a closed, metrizable subset of an letrs $X$. Let $C \subset Y$ be a finite union of closed, convex sets $C_{i}, C=\bigcup_{i=1}^{m} C_{i}$. Then there exists a compact, finite dimensional (i.e., its range lies in a finite dimensional subspace of $X$ ) map $R: C \rightarrow C$ such that $R(x) \in C_{i}$ if $x \in C_{i}$.

Proof. - For each $J C\{1,2, \ldots, m\}$ such that $C_{J}$ is nonempty, select $x_{j} \in C_{J}$. For $1 \leq j \leq m$, let $D_{j}=\operatorname{co}\left\{x_{J} \mid j \in J\right\}$, where co(S) denotes the convex hull of a set $S$. Since $D_{j}$ is the continuous image of the compact set of ordered $N$-tuples $\left\{\left(\lambda_{L}\right) \mid L \subset\{1,2, \ldots, m\}, C_{L} \neq \varnothing, j \in L, \sum_{L} \lambda_{L}=1\right\}$ (where $N$ denotes the number of subsets $L \subset\{1,2, \ldots, m\}$ such that $j \in L$ and $C_{L}$ is nonemptyl ander the map $\left(\lambda_{L}\right) \rightarrow \sum_{L} \lambda_{L} x_{L}, D_{J}$ is compact. It is clear that $D_{j} \subset C_{j}$ and that $\bigcap_{j \in J} D_{j}$ is nonempty iff $\bigcap_{j \in J} C_{j}$ is nonempty for all $J \subset$ $\{1,2, \ldots, m\}$. It follows by Theorem 1 that there exists a retraction $R: C$ $\rightarrow D=\bigcup_{i=1}^{m} D_{j}$ and $R(x) \in C_{j}$ if $x \in C_{j} . R$ is obviously compact and finite dimensional.

Q.E.D.

Corollary 2. - Let $Y$ be a closed, completely metrizable subset of an letrs $X$. Let $A_{n}^{=} \bigcup_{i=1}^{m} C_{i, n}$ be a union of $m$ bounded, closed, convex subsets of $Y, m$ independent of $n$. Assume that $C_{i, n} \supset C_{i n+1}$ for $1 \leq i \leq m, 1 \leq n \leq \infty$ and suppose that $\gamma\left(A_{n}\right) \rightarrow 0$, where $\gamma$ denotes the measure of noncompactness with respect to a complete metric $d$ for $Y$. Let $A_{\infty}=\bigcap_{n \geq 1} A_{n}$. Then there exists $n_{0} \geq 1$ such that for $n \geq n_{0}$ there exists a retraction $R_{n}: A_{n} \rightarrow A_{\infty}$ such that $R_{n}(x) \in C_{i, n}$ if $x \in C_{i, n}$. If $p$ is any continuous seminorm and $\varepsilon>0$, there exists an integer $n_{1}$ such that for $n \geq n_{1}$ the $R_{n}$ can be chosen so that $p\left(R_{n}(x)-x\right)<\varepsilon$.

Proof. - If we write $C_{j, \infty}=\bigcap_{n \geq 1} C_{i, n}$, it is easy to see that $A \overline{\bar{\infty}} \bigcup_{i=1}^{n} C_{i, \infty}$. Also, if for $J \subset\{1,2, \ldots, m\}$ we put $C_{J, \infty}=\bigcap_{j \in J} C_{j, \infty}$ and $C_{J, n}=\bigcap_{j \in J} C_{J, n}$, $C_{J, \infty}=\bigcap_{n \geq 1} C_{J, n}$. In order to prove the first part of the Corollary, it suffices 
by Theorem 1 to find an integer $n_{0}$ such that for $n \geq n_{0}, C_{J, \infty}$ is empty iff $C_{J, n}$ is empty. However, $C_{J, n}$ is a decreasing sequence of elosed sets in a complete metric space $Y, \gamma\left(C_{J, n}\right) \rightarrow 0$ and $C_{J,} \overline{\bar{\infty}} \bigcap_{n \geq 1} C_{J, n}$. It follows by Kuratowski's theorem that if $C_{J, \infty}$ is empty, $C_{J, n}$ is empty for $n \geq n_{J}$. Selecting $n_{0} \geq \max \left\{n_{J}\right\}$, we are done.

To prove the second part of the Corollary, let $\tilde{O}_{K}$ be the open neighborhood of $C_{K, \infty}$ guaranteed by Theorem 1. By Theorem 1, it suffices to find an integer $n_{0} \geq N_{0}$ such that $C_{K,{ }_{n}} \subset \tilde{O}_{K}$ for $n \geq n_{1}$. However, by Kuratowski's theorem again, there exists an integer $n_{K}$ such that $C_{K, n} \subset \tilde{O}_{K}$ for $n \geq n_{K}$. We merely take $n_{1} \geq \max \left\{n_{0}, n_{K}\right\}$.

Q.E.D.

\section{0. - The classical fixed point index.}

Our goal in the next few sections is to define a fixed point index for $k$-set-contraction, $k<1$, defined on certain «nice» absolute neighborhood retracts (ANR's). Let us begin by recalling the basic properties of the fixed point index [6]. Let $a$ be the category of compact metric absolute neighborhood retracts (ANR's) and continuous mappings. Let $A$ belong to $\alpha, G$ be an open subset of $A$, and $f: \operatorname{cl}(G) \rightarrow A$ be a continuous function which has no fixed points on $\operatorname{cl}(G)-G$. Then there is a unique integer valued function $i_{A}(f, G)$ which satisfies the following four properties:

1. - If $f: \operatorname{cl}(G) \rightarrow A$ has no fixed point on $\operatorname{cl}(g)-G$, and the fixed point of $f$ in $G$ lie in $G_{1} \cup G_{2}$, where $G_{1}$ and $G_{2}$ are two disjoint open sets included in $G$, then $i_{A}(f, G)=i_{A}\left(f, G_{1}\right)+i_{A}\left(f, G_{2}\right)$. In particular, if $f$ has no fixed points in $G$, this is meant to say that $i_{A}(f, G)=0$. (The additivity property).

2. - Let $I$ denote the clooed unit interval $[0,1]$. If $F: \operatorname{cl}(G) \times I \rightarrow A$ ( $A$ belongs to $a$, of course) is a continuous map, and $F_{t}(x)=F(x, t)$ has no fixed points on $\operatorname{cl}(G)-G$ for $0 \leq t \leq 1$, then $i_{A}\left(F_{0}, G\right)=i_{A}\left(F_{1}, G\right)$. (The ho* motopy propertyl.

3. - If $G=A$, then $i_{A}(f, G)=\Delta(f) . \Delta(f)$, the LEFSCHETz number of $f$, equals $\Sigma(-1)^{k}$ trace $\left(f_{*_{k}}\right)$, where $f_{*_{k}}: H_{k}(A) \rightarrow H_{k}(A)$ is the vector space homomorphism of $H_{k}(A)$ to $H(A)$ and $H_{k}(A)$ is the Cech homology of $A$ with rational coefficients. (The normalization property).

4. - Let $A$ and $B$ be two spaces which belong to $a$. Let $f: A \rightarrow B$ be a continuous map. Let $V$ be an open subset of $B$ and $g: \operatorname{cl}(\nabla) \rightarrow A$ a continuous map. Assume $f g$ has no fixed points on $\operatorname{cl}(V)-V$. Let $U=f^{-1}(V)$. Then $g f$ has no fixed points on $\mathrm{cl}(U)-U$ and $i_{B}(f g, V)=i_{A}(g f, U)$. (The commutativity property). 
As a special case of Property 4, we have the following property, which we shall use repeatedly:

4'. - Let $A$ and $B$ belong to $a, A \subset B$. Let $f: A \rightarrow B$ be the inclusion. Let $G$ be and open subset of $B$ and $g: \operatorname{cl}(G) \rightarrow A$ be a continuous map with no fixed points on $\operatorname{cl}(G)-G$. Then we have $i_{B}(f g ; G)=i_{B}(g, G)=i_{A}\left(g f, f^{-1}\right.$ $(G))=i_{A}(g, G \cap A)$.

Let us begin our generalizations with a slight widening of the definition of the fixed point index. Let $A$ belong to $a$ and let $G$ be an open subset of $A$. Let $g: G \rightarrow A$ be a continuous map and assume that $S=\{x \in G \mid g(x)=x\}$ is a compact subset of $G$. ( $S$ may be empty. By compact we shall always mean compact or empty!. Clearly we can select an open neighborhood $V$ of $S$ such that $\operatorname{cl}(V) \subset G$. We define $i_{A}(g, G)=i_{A}(g, V)$. We have to show that this definition does not depend on the particular $V$ chosen and agrees with our previous definition when $g$ is defined on $\operatorname{cl}(G)$. To see that the definition is independent of $V$, let $V_{1}$ and $\nabla_{2}$ be two open neighborhoods of $S$ with $\operatorname{cl}\left(V_{i}\right) \subset G$. Then $\nabla_{1} \cap \nabla_{2}$ is an open neighborhood of $S$ and $\operatorname{cl}\left(\nabla_{1} \cap \nabla_{2}\right) \subset G$. If we consider the disjoint open sets $\nabla_{i} \sim \operatorname{cl}\left(\nabla_{1} \cap \nabla_{2}\right)$ and $\nabla_{1} \cap \nabla_{2}$, since $S \subset \nabla_{1} \cap V_{2}$ it is clear that the additivity property applies and $i_{A}\left(g, V_{i}\right)=$ $=i_{A}\left(g, V_{i} \sim \operatorname{cl}\left(V_{1} \cap V_{2}\right)\right)+i_{A}\left(g, \nabla_{1} \cap V_{2}\right)$. Since $f$ has no fixed points in $\nabla_{i} \sim \operatorname{ol}\left(\nabla_{1} \cap \nabla_{2}\right), i_{A}\left(g, \nabla_{i} \sim \operatorname{cl}\left(\nabla_{1} \cap \nabla_{2}\right)\right)=0$ and $i_{A}\left(g, \nabla_{1}\right)=i_{A}\left(g, V_{1} \cap V_{2}\right)=i_{A}\left(g, \nabla_{2}\right)$. If $g$ is defined on $\operatorname{cl}(G)$ and $\nabla$ is selected as above, $\left.i_{A}(g, G)=i_{A} g, G-\operatorname{cl}(V)\right)=$ $=i_{A}(g, V)$. Thus our definition agrees with the usual one when $g$ is defined on $\operatorname{cl}(G)$.

We want to establish generalisations of the four properties listed above.

Theonem 1. - Let $A$ belong to $a, G$ be an open subset of $A$, and $f: G \rightarrow A$ be a continuous map. Assume that $S=\{x \in G \mid f(x)=x\}$ is a com. pact subset of $G$ and that $S \subset G_{1} \cup G_{2}$ where $G_{i}$ are disjoint open subsets of $G$. Then $i_{A}(f, G)=i_{A}\left(f, G_{1}\right)+i_{A}\left(f, G_{2}\right)$.

Proof. - Notice that $S_{1}=\left\{x \in G_{1}: f(x)=x\right\}$ is a compact subset of $G_{1}$, because $S_{1}=\left\{x: x \in S \cap\left(A \sim G_{2}\right)\right\}$. Similarly, $S_{2}=\left\{x \in G_{2}: f(x)=x\right\}$ is com. pact. Thus we see $i_{A}\left(f, G_{i}\right)$ is defined.

Let $V_{i}$ be an open neighborhood of $S_{i}$ such that $\operatorname{cl}\left(\nabla_{i}\right) \subset G_{i}$. Since $G_{3}$ and $G_{2}$ are disjoint, $V_{1}$ and $V_{2}$ are disjoint. Let $V=V_{1} \cup V_{2} . V$ is an open neighborhood of $S$, and $\operatorname{cl}(V)=\operatorname{cl}\left(V_{1}\right) \cup \operatorname{cl}\left(V_{2}\right) \subset G$. By the ordinary additivity property, we have $\left.i_{A}\left(f, G_{1}\right)+i_{A}\left(f, G_{2}\right) \equiv i_{A}\left(f, \nabla_{1}\right)+i_{A}\left(f, \nabla_{2}\right)=i_{A}(f, V) \equiv i_{A} f, G\right)$.

Theorem 2. - Let $A$ belong to $a$ and let $I=[0,1]$. Let $\Omega$ be an open subset of $A \times I$ and $F: \Omega \rightarrow A$ a continuons map. Assume that $S=\{(x, t) \in$ $\in \Omega: F(x, t)=x\}$ is compact. Let $\Omega_{t}=\{x \in A:(x, t) \in \Omega\}$ and $F_{t}=(F \cdot, t)$. Then we have $i_{A}\left(F_{0}, \Omega_{0}\right)=i_{A}\left(F_{1}, \Omega_{1}\right)$. 
REMaRK. - Notice that if $\Omega=G \times I$ and $F$ is a continuous function from $\operatorname{cl}(G) \times I$ to $A$ such that $F(x, t) \neq x$ for $x \in \operatorname{cl}(G) \sim G$, then $S=\{(x, t) \in$ $\in G \times I: F(x, t)=x\}=\{(x, t) \in \operatorname{cl}(G) \times I: F(x, t)=x\}$ is compact.

Proor. - Suppose we can show that every $t \in I$ has an open neighbor. hood (open in $I$ ), $0_{t}$, such that $i_{d}{ }_{i} F_{s}, \Omega_{s}$ ) is constant for $s \in 0_{l}$. If we let $U=\left\{t \in I: i_{A}\left(F_{t}, \Omega_{t}\right)=i_{A}\left(F_{0}, \Omega_{0}\right)\right\}$, it then follows that $U$ is open in $I$. But $U$ is the complement in $I$ of $V=\left\{t \in I: i_{A}\left(F_{t}, \Omega_{t}\right) \neq i_{A}\left(F_{0}, \Omega_{0}\right)\right\}$, and the same reasoning implies that $V$ is open. Thus $U$ is open and closed and hence $U=1$.

Thus we have only to show that given $t_{0} \in I$, there is an open neighbor. hood $0_{t_{0}}$ such that $i_{A}\left(F_{t}, \Omega_{t}\right)$ is constant for $t \in 0_{t_{0}}$. Let $S_{t}=\{(x, t) \mid(x, t) \in S\}$. Given $\left(x, t_{0}\right) \in S_{t_{0}}$, we can find an open neighborhood $N_{x} \times\left(J_{x} \cap I\right)$ of $\left(x_{0}, t_{0}\right)$ (open in the topology of $\Omega$ ) such that $\operatorname{cl}\left(N_{x}\right) \times \operatorname{cl}\left(J_{x} \cap I\right) \subset \Omega, N_{x}$ is an open neighborhood in $A$ of $x$ and $J_{x}=\left(t_{0}-\varepsilon_{x}, t_{0}+\varepsilon_{x}\right)$ is an open interval about $t_{0}$. Since $S_{t_{0}}$ is compact, we can over it by a finite number of these neighborhoods, say $N_{x_{i}} \times\left(J_{x_{i}} \cap l\right), 1 \leq i \leq n$. Let $\varepsilon=\min \left\{\varepsilon_{x_{i}}, 1 \leq i \leq n\right\}$, let $V=$ $=\bigcup_{i=1}^{n} N_{x_{i}}$, and let $J=\left(t_{0}-\varepsilon, t_{0}+\varepsilon\right)$. Then $S_{t_{0}} \subset V \times(J \cap I)$ and $\operatorname{cl}(\nabla) \times$ $\times \operatorname{cl}(J \cap I) \subset \Omega$.

Let $J_{\eta}=\left(t_{0}-\eta, t_{0}+\eta\right)$. We claim that for $\eta$ small enough, $S_{t} \subset V \times$ $\left.\times \operatorname{cl}_{(} J_{\eta} \cap I\right)$ for $t \in \operatorname{cl}\left(J_{\eta} \cap I\right)$. The proofs is by contradiction. Suppose not. Then we can find $\left(x_{n}, t_{n}\right) \in S$ such that $t_{n} \rightarrow t_{0}$ but $x_{n} \notin V$. Since $S$ is compact, we can find a convergent subsequence $\left(x_{n_{i}}, t_{n_{i}}\right) \rightarrow\left(x, t_{0}\right)$. By the continuity of $F, F\left(x, t_{0}\right)=x$, so $\left(x, t_{0}\right) \in S_{t_{0}}$. But $x_{n_{i}} \notin V$, so $x \notin V$ since $V$ is open. This is a contradiction.

We are almost finished. Select $V$ and $\eta$ as above. $F$ is a continuous function on $\operatorname{cl}(V) \times \operatorname{cl}\left(J_{\eta} \cap I\right)$, and, of oourse, $\operatorname{cl}\left(J_{\eta} \cap I\right)$ is a closed interval of real numbers. By construction we have $F(x, t) \neq x$ for $x \in \operatorname{cl}(V)-V$ and $t \in \operatorname{cl}\left(J_{\eta_{i}} \cap I\right)$. Thus, by the ordinary homotopy property we find $i_{A}\left(F_{t}, V\right)$ is constant for $t \in \operatorname{cl}\left(J_{\eta} \cap I\right)$. However, for $t \in \operatorname{cl}\left(J_{n} \cap I\right), V$ is an open neighborhood of $\left\{x \mid F_{t}(x)=x\right\}=\left\{x \mid(x, t) \in S_{t}\right\}$, and we know that $\operatorname{cl}(\nabla) \subset \Omega_{t}$. Thus we have $i_{A}\left(F_{t}, V\right)=i_{A}\left(F_{t}, \Omega_{t}\right)$.

Q.E.D.

The normalization property in our new context is the same as before, and we do not repeat its statement.

Thr proof of the generalized version of the multiplication formula is somewhat more involved than the previous results. First we need a few simple lemmas.

Lemas 1. - Let $G_{i}$ be an open subset of a topological space $A_{i}, i=1,2$. Let $f_{1}: G_{1} \rightarrow A_{2}$ and $f_{2}: G_{2} \rightarrow A_{1}$ be continuous maps. Let $S_{1}=\left\{x \in f_{1}^{-1}\left(G_{2}\right)\right.$ : $\left.\left(f_{2} f_{1}\right)(x)=x\right\}$ and let $S_{2}=\left\{x \in f_{2}^{-1}\left(G_{1}\right):\left(f_{1} f_{2}\right)(x)=x\right\}$. Then $f_{1}$ takes $S_{1}$ into $S_{2}$ 
and $f_{1}$ restricted to $S_{1}$ is a homeomorphism of $S_{1}$ onto $S_{2}$ whose inverse is $f_{2}$ restricted to $S_{2}$. In particular, $S_{1}$ is compact iff $S_{2}$ is compact.

Proor. - If $x \in S_{1},\left(f_{2} f_{1}\right)(x)=x$ so $\left(f_{1} f_{2}\right)\left(f_{1}(x)\right)=f_{1}(x)$ and $f_{1}(x) \in S_{2}$. Conver. sely, if $y \in S_{2},\left(f_{1} f_{2}\right)(y)=y$, so $\left(f_{2} f_{1}\right)\left(f_{2}(y)\right)=f_{2}(y)$ and $f_{2}(y) \in S_{1}$. Thus we have $S_{1} \stackrel{f_{1}}{\rightarrow} S_{2} \stackrel{f_{2}}{\rightarrow} S_{1}$. However, if $x \in S_{1},\left(f_{2} f_{1}\right)(x)=x$, and if $y \in S_{2},\left(f_{2} f_{1}\right)(y)=y$, so $f_{1} f_{2}$ is the identity on $S_{1}$ and $f_{1} f_{2}$ is the identity on $S_{2}$ and $f_{1} f_{2}$ is the identity on $S_{2}$, i.e., $f_{1} \mid S_{1}$ is a homeomorphism.

Q.E.D.

Our next lemma is a standard result, but we include the proof for completeness.

Lemma 2. - Any complete metric space $(X, d)$ cnn be isometrically imbedded as a closed subset of BANach space $B$.

Proof. - Consider the space $B$ of real valued, bounded, continuous functions on $X$. If $f \in B,\|f\|=\sup _{x \in X}|f(x)|$. Take a fixed point $x_{0} \in X$, and for $x \in X$, consider the function $f_{x} \in B$ given by $f_{x}(y)=d(y, x)-d\left(y, x_{0}\right)$. First, notice that $f_{x}$ is bounded, for $\left.\mid f_{x}(y)\right\} \leq d\left(x, x_{0}\right)$. Clearly $f_{x}$ is continuous. We now claim that the map $j: x \rightarrow f_{x}$ is an isometry. For, take $x_{1}$ and $x_{2} \in X$ and consider $\left\|f_{x_{1}}-f_{x_{2}}\right\|$. For $y \in X$ we have that $\left|f_{x_{1}}(y)-f_{x_{2}}(y)\right|=\mid d\left(y, x_{1}\right)-d\left(y, x_{0}\right)-d\left(y, x_{2}\right)$ $+d\left(y, x_{0}\right)|=| d\left(y, x_{1}\right)-d\left(y, x_{2}\right) \mid \leq d\left(x_{1}, x_{2}\right)$ so $\left\|f_{x_{1}}-f_{x_{2}}\right\| \leq d\left(x_{1}, x_{2}\right)$. Conversely, if we take $y=x_{2}$ we see that $\left|f_{x_{1}}(y)-f_{x_{2}}(y)\right|=\mid d\left(x_{2}, x_{1}\right)-d\left(x_{2}, x_{0}\right)-$ $\left.-d\left(x_{2}, x_{2}\right)+d\left(x_{2}, x_{0}\right)\right)=d\left(x_{1}, x_{2}\right)$, so $\left\|f_{x_{1}}-f_{x_{2}}\right\| \geq d\left(x_{1}, x_{2}\right)$.

Finally, notice that $j X$ is a closed subset of $B$. Eor suppose that $f_{x} \rightarrow g$. Then $f_{x_{n}}$ is a CAUany sequence, so $x_{n}$ is a CAUCHY sequence. Since $X$ is complete, $x_{n} \rightarrow x$ and consequently $f_{x_{n}} \rightarrow f_{x}=g$.

Q.E.D.

Lemma 3. - Let $A$ be a compact metric ANR and let $h: A \rightarrow A_{1}$ be a homeomorphism of $A$ onto a topological space $A_{1}$. Let $G$ be an open subset of $A$ and $f: G \rightarrow A$ a continuous map such that $S=\{x \in G: f(x)=x\}$ is compact. Let $G_{1}=h(G)$ and $f_{1}=h f h^{-1}: h\left(G_{1}\right) \rightarrow A_{1}$. Then $i_{A}(f, G)=i_{A_{1}}\left(f_{3}, G_{1}\right)$.

Proof. - Notice that $i_{A_{1}}\left(f_{1}, G_{1}\right)$ is defined, since $S_{1}=\left\{y \in G_{1}: f_{1}(y)=y\right\}=h(S)$. Let $V$ be an open neighborhood of $S$ such that $\operatorname{cl}(V) \subset G$ and let $\nabla_{1}=h(V)$. By definition we have $i_{A}(f, G)=i_{A}(f, \nabla)$ and $i_{A_{1}}\left(f_{1}, G_{1}\right)=i_{A_{1}}\left(f_{1}, V_{1}\right)$. To show $i_{A}(f, V)=i_{A}\left(f_{1}, \nabla_{1}\right)$ we use the commutativity property. Since $f_{1}=h\left(f h^{-1}\right) \equiv h g$ and $h: A \rightarrow A_{1}$ while $g: V_{1} \rightarrow A$, commutativity implies $i_{A_{1}}\left(h g, V_{1}\right)=i_{A}\left(g h, h^{-1}\right.$ $\left.\left(\nabla_{1}\right)\right)=i_{A}(f, \nabla)$.

Q.E.D.

Lemma 4. - Let $A_{1}$ and $A_{2}$ be finite unions of compact, convex sets in BANACH space $X_{1}$ and $X_{2}$ respectively. It is known then that $A_{1}$ and $A_{2}$ are compact metric ANR's. Let $0_{1}$ and $0_{2}$ be open subsets of $A_{1}$ and $A_{2}$ respectively and let $f_{1}: 0_{1} \rightarrow A_{2}$ and $f_{2}: 0_{2} \rightarrow A_{1}$ be continuous maps. Assume that 
the set of fixed points of $f_{2} f_{1}$ in $f_{1}^{-1}\left(0_{2}\right)$ is a compact subset of $f_{1}^{-1}\left(0_{2}\right)$. Then $i_{A_{1}}\left(f_{2} f_{1}, f_{1}^{-1}\left(O_{2}\right)\right)=i_{A_{2}}\left(f_{1} f_{2}, f_{2}^{-1}\left(O_{1}\right)\right)$.

REMaRK. - The fact that $A_{i}$ is an ANR follows from two theorems. First, by a theorem of DUGUNDJI [15], a closed, convex subset of a BANACH space is an AR. Second, if two topological spaces (both contained in some topolo. gical space) are ANRs and their intersection is also an ANR, then their union is an ANR.

Proof. - By Lemma 1, the fixed point set of $f_{1} f_{2}$ in $f_{2}^{-1}\left(0_{1}\right)$ is a compact subset of $f_{2}^{-1}\left(0_{1}\right)$, so $i_{A_{2}}\left(f_{1} f_{2}, f_{2}^{-1}\left(0_{1}\right)\right)$ is defined. Let $S_{2}$ denote the fixed point sel of $f_{1} f_{2}$ in $f_{2}^{-1}\left(0_{1}\right)$ and recall that $f_{2} . S_{2} \rightarrow S_{1}$ is a homeomorphism.

Let $U_{2}$ be an open neighborhood of $S_{2}$ such that $\operatorname{cl}\left(U_{2}\right) \subset f_{2}^{-1}\left(0_{1}\right)$. Let $U_{1}$ be an open neighborhood of $S_{1}$ such that $\operatorname{cl}\left(U_{1}\right) \subset f_{1}^{-1}\left(0_{2}\right)$ and $\operatorname{cl}\left[f_{1}\left(U_{1}\right)\right] \subset 0_{2}$. To obtain such a $U_{1}$ we simply take an open neighborhood $\nabla$ of $S_{2}$ such that $\mathrm{cl}(V) \subset 0_{2}$, and we put $U_{1}=f_{1}^{-1}(V)$. Since $\mathrm{cl}\left[f_{1}\left(U_{1}\right)\right]$ is a compact subset of $0_{2}$, we can cover it by a finite number of balls in $X_{2}$, say $B_{1}, \ldots, B_{n}$ such that $\operatorname{cl}\left(B_{i} \cap A_{2}\right) \subset 0_{2}, 1 \leq i \leq n$. Since $A_{2}$ is a finite union of compact, convex pieces, el $\left(B_{i} \cap A_{2}\right)$ is a finite union of compact, convex pieces, and consequently so is $A=\bigcup_{i=1}^{n} \mathrm{cl}\left(B_{i} \cap A_{2}\right)$.

With these preliminary constructions, we ean complete our proof. By definition, $i_{A_{1}}\left(f_{2} f_{1}, f_{1}^{-1}\left(0_{2}\right)\right)=i_{A_{1}}\left(f_{2} f_{1}, U_{1}\right)$. On the other hand, by construction, $f_{1}\left(U_{1}\right) \subset A$; and since $A \subset 0_{2}, f_{2}$ is defined on $A$ and $f_{2}: A \rightarrow A_{1}$. Thus we can apply the ordinary commutativity property, and we obtain $i_{A}\left[f_{1} f_{2},\left(f_{2} \mid A\right)^{-1}\right.$ $\left.\left(U_{1}\right)\right]=i_{A_{1}}\left(f_{2} f_{1}, U_{1}\right)$. We now have to show that $i_{A}\left[f_{1} f_{2},\left(f_{2} \mid A\right)^{-1}\left(U_{1}\right)\right]=i_{A_{2}}\left(f_{1} f_{2}, U_{2}\right)$. If we can show this, we are done, for by definition $i_{A_{2}}\left(f_{1} f_{2}, U_{2}\right)=i_{A_{2}}\left(f_{1} f_{2}, f_{2}^{-1}\left(0_{1}\right)\right)$. Thus, suppose that $\left(f_{1} f_{2}\right)(x)=x$ for $x \in f_{2}^{-1}\left(0_{1}\right)$, i.e., $x \in S_{2}$. Since $f_{2}: S_{2} \rightarrow S_{1}$ is a homeomorphism, $x=f_{2}^{-1}(z)$ for some $z \in S_{1} \subset U_{1}$; in other words, we have $S_{2} \subset f_{2}^{-3}\left(U_{1}\right)$. It follows by the additivity property that $i_{A_{2}}\left(f_{1} f_{2}, U_{2}\right)=i_{A_{2}}\left(f_{1} f_{2}\right.$, $\left.f_{2}^{-1}\left(U_{1}\right) \cap U_{2}\right)$. However, $f_{1} f_{2}: f_{2}^{-1}\left(U_{1}\right) \cap U_{2} \rightarrow A \subset A_{2}$, so by Property $4^{\prime}, i_{A_{1}}\left(f_{1} f_{2}\right.$, $\left.f_{2}^{-1}\left(U_{1}\right) \cap U_{2}\right)=i_{A}\left(f_{1} f_{2}, f_{2}^{-1}\left(U_{1}\right) \cap U_{2} \cap A\right)$. We see easily, however, that $f_{2}^{-1}\left(U_{1}\right) \cap$ $\cap A=\left(f_{2} \mid A\right)^{-1}\left(U_{1}\right)$, and since $S_{2} \subset U_{2} \cap\left(f_{2} \mid A\right)^{-1}\left(U_{1}\right)$, the additivity property implies that $i_{A}\left(f_{1} f_{2},\left(f_{2} \mid A\right)^{-1}\left(U_{1}\right)\right)=i_{A}\left(f_{1} f_{2}, f_{2}^{-1}\left(U_{1}\right) \cap U_{2} \cap A\right) . \quad$ Q.E.D.

Theonem 3. - Let $A_{1}$ and $A_{2}$ be compact metric ANR's, $0_{1}$ and $0_{2}$ open subsets of $A_{1}$ and $A_{2}$ respectively, and $f_{1}: 0_{1} \rightarrow A_{2}$ and $f_{2}: O_{2} \rightarrow A_{1}$ continuous maps. Assume that $S_{1}=\left\{x \in f_{1}^{-1}\left(0_{2}\right):\left(f_{2} f_{1}\right)(x)=x\right\}$ is compact. Then $i_{A_{2}}\left(f_{1} f_{2}\right.$, $\left.f_{2}^{-1}\left(0_{1}\right)\right)$ is defined and $i_{A_{2}}\left(f_{1} f_{2}, f_{2}^{-1}\left(0_{1}\right)\right)=i_{A_{1}}\left(f_{2} f_{1}, f_{1}^{-1}\left(0_{2}\right)\right)$.

Proof. - By Lemma 2 there exist isometric imbeddings $j_{1}$ and $j_{2}$ of $A_{1}$ and $A_{2}$ respectively into $B_{\text {ANACH }}$ spaces $X_{1}$ and $X_{2}$. If we set $U_{i}=j_{i}\left(0_{i}\right)$, $D_{i}=j_{i}\left(A_{i}\right), \quad(i=1,2), g_{1}=j_{2}^{-1} f_{1} j_{2}^{-1}$ and $g_{2}=j_{1} f_{2} j_{2}^{-1}$, and if we note that $g_{1}^{-1}\left(U_{2}\right)=j_{1}\left(f_{1}^{-1}\left(0_{2}\right)\right)$, then Lemma 3 implies that $i_{A_{1}}\left(f_{2} f_{1}, f_{1}^{-1}\left(0_{2}\right)\right)=i_{D_{1}}\left(j_{1}\left(f_{2} f_{1}\right) j_{1}^{-1}\right.$, 
$\left.j_{1}\left(f_{1}^{-1}\left(O_{2}\right)\right)\right)=i_{D_{1}}\left(g_{2} g_{1}, g_{1}^{-1}\left(U_{2}\right)\right)$ and similarly for $A_{2}$ and $D_{2}$. This implies that in proving Theorem 3 we can assume $A_{1}$ and $A_{2}$ are subsets of B BNACI spaces $X_{1}$ and $X_{2}$ respectively.

Since $A_{i}$ is an ANR, $A_{i}$ is a retract of some open neighboorhood $U_{i}$ in $X_{i}$ by a retraction $r_{i}: U_{i} \rightarrow A_{i}, i=1,2$. Cover $A_{i}$ by a finite number of open balls $B_{i j}, 1 \leq j \leq k_{i}$, such that $\operatorname{cl}\left(B_{i j}\right) \subset U_{i}, i=1$, 2. Then we see that $\cup_{j=1}^{k_{i}} \overline{c o}\left(B_{i j} \cap A_{i}\right) \equiv B_{i}$ is a finite union of compact, oonvex sets, $B_{i} \supset A_{i}$, and $r_{i}: B_{i} \rightarrow A_{i}$. Henceforth we shall view the maps $r_{i}$ as defined only on $B_{i}$.

We have $f_{1} r_{1}: r_{1}^{-1}\left(0_{1}\right) \rightarrow A_{2} \subset B_{2}$ and $f_{2} r_{2}: r_{2}^{-1}\left(0_{1}\right) \rightarrow A_{1} \subset B_{1}$. If $\tilde{S}_{1}=\{x \in$ $\left.\epsilon\left(f_{1} r_{1}\right)^{-1}\left(r_{2}^{-1}\left(0_{2}\right)\right):\left(f_{2} r_{2}\right)\left(f_{1} r_{2}\right)(x)=x\right\}$, we easily check that $\tilde{S}_{1}=\left\{x \in r_{1}^{-1}\left(f_{1}^{-1}\left(0_{2}\right)\right)\right.$ : $\left.\left(f_{2} r_{2}\right)\left(f_{1} r_{1}\right)(x)=x\right\}=\left\{x \in f_{1}^{-1}\left(0_{2}\right):\left(f_{2} f_{1}\right)(x)=x\right\}=S_{1}$, so that $\tilde{S}_{1}$ is a compact subset of $\left(f_{1} r_{1}\right)^{-1}\left(r_{2}^{-1}\left(0_{2}\right)\right)=r_{1}^{-1}\left(f_{1}^{-1}\left(0_{2}\right)\right)$.

Similary, we check that $\tilde{S}_{2}=\left\{x \in\left(f_{2} r_{2}\right)^{-1}\left(r_{1}^{-1}\left(0_{1}\right)\right):\left(f_{1} r_{1}\right)\left(f_{2} r_{2}\right)(x)=x\right\}$ is equal to $S_{2}$ and is a compact subset of $r_{2}^{-1}\left(f_{2}^{-1}\left(0_{1}\right)\right)$. Applying Lemma 4 we find that $i_{B_{1}}\left[\left(f_{2} r_{2}\right)\left(f_{1} r_{1}\right),\left(f_{1} r_{1}\right)^{-1}\left(r_{2}^{-1}\left(0_{2}\right)\right)\right]=i_{B_{2}}\left[\left(f_{1} r_{1}\right)\left(f_{2} r_{2}\right),\left(f_{2} r_{2}\right)^{-1}\left(r_{1}^{-1}\left(0_{1}\right)\right)\right]$. However, it is easy to see that $\left(f_{2} r_{2}\right)\left(f_{1} r_{1}\right)=\left(f_{2} f_{1}\right)\left(r_{1}\right),\left(f_{1} r_{1}\right)\left(f_{2} r_{2}\right)=\left(f_{1} f_{2}\right)\left(r_{2}\right),\left(f_{1} r_{1}\right)^{-1}\left(r_{2}^{-1}\left(0_{2}\right)\right)=$ $=\left(r_{1}^{-1}\left(f_{1}^{-1}\left(0_{2}\right)\right)\right.$ and $\left(f_{2} r_{2}\right)\left(r_{1}^{-1}\left(0_{1}\right)=r_{2}^{-1}\left(f_{2}^{-1}\left(0_{1}\right)\right)\right.$. Substituting these results in the above equality, we obtain $i_{B_{1}}\left[\left(f_{2} f_{1}\right)\left(r_{1}\right), r_{1}^{-1}\left(f_{1}^{-1}\left(0_{2}\right)\right)\right]=i_{B_{2}}\left[\left(f_{1} f_{2}\right)\left(r_{2}\right), r_{2}^{-1}\left(f_{2}^{-1}\left(0_{1}\right)\right)\right]$.

Now we apply the ordinary commutativity property again:

We have $r_{1}: B_{1} \rightarrow A_{1},\left(f_{2} f_{1}\right): f_{1}^{-1}\left(0_{2}\right) \rightarrow A_{1} \subset B_{1}$, and the other necessary conditions are met, so $i_{A_{1}}\left[\left(r_{1}\right)\left(f_{2} f_{1}\right), f_{1}^{-1}\left(0_{2}\right)\right]=i_{A_{1}}\left(f_{2} f_{1}, f_{1}^{-1}\left(0_{2}\right)\right)=i_{B_{1}}\left[\left(f_{2} f_{1}\right)\left(r_{1}\right)\right.$, $\left.r_{1}^{-1}\left(f_{1}^{-1}\left(0_{2}\right)\right)\right]$. Similarly, we obtain $i_{A_{2}}\left(f_{1} f_{2}, f_{2}^{-1}\left(0_{1}\right)\right)=i_{B_{3}}\left[\left(f_{2} f_{1}\right)\left(r_{2}\right), r_{2}^{-1}\left(f_{2}^{-1}\left(0_{1}\right)\right)\right]$.

Q.E.D.

We have now obtained the desired refinements of the fundamental properties of the fixed point index. We next establish some special properties which will be crucial for our further work.

Let us begin by considering the following general situation: Let $A=A_{1}$, $A_{2}, \ldots, A_{n}, \ldots$ be a decreasing sequence of compact ANR's in a B BNACH space (we allow the possibility that the $A_{n}{ }^{\prime} s$ are empty for $n \geq n_{0}$ ). Assume that $A_{\infty}=\bigcap_{n \geq 1} A_{n}$ is an ANR. Let $G$ be an open subset of $A$ and $f: \operatorname{cl}(G) \rightarrow A$ a continuous map such that $f(x) \neq x$ for $x \in \operatorname{cl}(G)-G$ and $f: G \cap A_{n} \rightarrow A_{n+1}$. By applying Property $4^{\prime}$ repeatedly, it is easy to show that $i_{A}(f, G)=$ $=i_{A_{n}}\left(f, G \cap A_{n}\right)$, It is thus natural to ask if $i_{A}(f, g)=i_{A_{\infty}}\left(f, G \cap A_{\infty}\right)$. We do not know exact]y when it is true that $i_{A}(f, G)=i_{A_{\infty}}\left(f, G \cap A_{\infty}\right)$. The following sufficient condition will be satisfactory for our purposes, however.

Lemma 5. - Let $A_{n}, f$, and $G$ be as above. Assume that for any $\delta>0$ there exists a sequence of deformation retractions $H_{n}: A_{n} \times I \rightarrow A_{n}$, defined for $n \geq n(\delta)$ and such that $H_{n}(\cdot, 0)$ is the identity on $A_{n}, H_{n}(\cdot, 1)$ is a retraction on $A_{\infty}, H_{n}(x, t)=x$ for $x \in A_{\infty}$ and $t \in I$, and $\left\|H_{n}(x, t)-x\right\|<\delta$ for $x \in A_{n}, t \in 1$. Then $i_{A}(f, G)=i_{A_{\infty}}\left(f, G \cap A_{\infty}\right)$. 
Remark. - The strength of the above assumption is that not only is $A_{\infty}$ a deformation retract of $A_{n}$ for $n$ large enough, but a deformation retraction $H_{n}$ can be chosen which moves points very little, i.e., $\left\|H_{n}(x, t)-x\right\|<\delta$ for $x \in A_{n}, t \in I$. If we assumed $f$ defined on all of $A_{1}$ and $f: A_{n} \rightarrow A_{n+1}$ (so that the various fixed point indices would be LEFscheTz numbers), then we would only need $A_{\infty}$ a deformation retract of $A_{n}$ for $n$ large enough in order to show $i_{A_{1}}\left(f, A_{1}\right)=\Lambda_{A_{1}}(f)=\Lambda_{A_{\infty}}(f)=i_{\Lambda_{\infty}}\left(f, A_{\infty}\right)$.

Proor. $-f(x) \neq x$ for $x \in \operatorname{cl}(G)-G$, and $\operatorname{cl}(G)-G$ is compact, so we can find $\delta>0$ such that $\|f(x)-x\| \geq \delta>0$ for $x \in \operatorname{cl}(G)-G$. Select $H_{n}$ as above for this $\delta$, so that $H_{n}$ is defined for $n \geq n(\delta)$ and $\left\|H_{n}(x, t)-x\right\|<\delta$ for $x \in A_{n}$, $t \in I$.

By using Property 4' repeatedly, we see that $i_{A_{1}}(f, G)=i_{A_{2}}\left(f, G \cap A_{2}\right)=$ $=i_{A_{n(\delta)}}\left(f, G \cap A_{n(\delta)}\right)$. (Notice that if $A_{n} \cap G$ is empty for some $n, f$ has no fixed points in $G$, since all fixed points lie in $A_{\infty} \cap G \subset A_{n} \cap G$. Thus in the case that $A_{n} \cap G$ is empty for some $n, i_{A_{m}}\left(f, A_{m} \cap G\right)=0$ for all $m$, and the lemma is proved). Consider the homotopy $F(x, t)=H_{n(\delta)}(f(x), t), F: A_{n(\hat{\delta})} \times$ $X I \rightarrow A_{n(\delta)}$. We have $\left\|H_{n(\delta)}(f(x), t) \rightarrow f(x)\right\|<\delta$ for $x \in \operatorname{cl}\left(G \cap A_{n(\delta)}\right)-G \cap A_{n(\delta)}$. It follows that $F(x, t) \neq x$ for $x \in \operatorname{cl}\left(G \cap A_{n(\delta)}\right)-G \cap A_{n(\delta)}$, so $i_{A_{n(\delta)}}(F(\cdot, 0)$, $\left.G \cap A_{n(\delta)}\right)=i_{A_{n}(\delta)}\left(f, G \cap A_{n(\delta)}\right)=i_{A_{n}(\delta)}\left(F(\cdot, 1), G \cap A_{n(\delta)}\right)$. However, $F(x, 1) \in A_{\infty}$ for $x \in G \cap A_{n(\delta)}$, so by Property $4^{\prime}, i_{A_{n(\delta)}}\left(F(\cdot, 1), G \cap A_{n(\delta)}\right)=i_{A_{\infty}}(F(\cdot, 1)$ $\left.\mid G \cap A_{\infty}, G \cap A_{\infty}\right)=i_{A_{\infty}}\left(f, G \cap A_{\infty}\right)$.

Q.E.D.

Of course the reason for proving Lemma 5 is that its hypotheses can be verified in a case which will be of interest to us.

Lemia 6. - Let $A_{n}=\bigcup_{:=1}^{m} C_{i, n}$ be a union of $m$ closed, convex sets in some fixed Banach space $X, m$ independent of $n$. Assume that $A_{n}$ is bounded, $\lim \gamma\left(A_{n}\right)=0$ and $C_{i, n} \supset C_{i, n+1}$ for $1 \leq i \leq m$. (We allow the possibility that $C_{i, n}$ is empty). Then for any $\delta>0$, there exists a sequence of deformation retractions $H_{n}: A_{n} \times I \rightarrow A_{n}$ defined for $n \geq n(\delta)$ and meeting the conditions of Lemma 5.

Proof, - By Corollary 2 of Section $B$, given $\delta>0$, there there exists $n(\delta)$ such that for $n \geq n(\delta)$ there exists a retraction $R_{n}: A_{n} \rightarrow A_{\infty}=\bigcap_{n \geq 1} A_{n}$ such that $\left\|R_{n}(x)-x\right\|<\delta$ for $x \in A_{n}$ and $R_{n}(x) \in C_{i, n}$ if $x \in C_{i, n}$. Set $H_{n}(x, t)=$ $=(1-t) x+t R_{n}(x)$. It is clear that $H_{n}$ satisfies the required conditions.

Q.E.D.

Theorem 4. - Let $A_{n}=\bigcup_{i=1}^{m} C_{i_{n},}$ be a union of $m$ compact, convex sets $C_{i, n}$ in a fixed BanaCr space $X, m$ independent of $n, 1 \leq n<\infty$. Assume that $C_{i, n} \supset C_{i, n+1}$ for $1 \leq n<\infty$. Let $G$ be an open subset of $A_{1}, f: G \rightarrow A_{1}$ a continuous map such that $S=\{x \in G: f(x)=x\}$ is compact. Assume that 
$f: G \cap A_{n} \rightarrow A_{n+1}$ for $1 \leq n<\infty$ and let $A_{\infty}=\bigcap_{n \geq 1} A_{n}$. Then $i_{A_{1}}(f, G)=$ $=i_{A_{\infty}}\left(f, G \cap A_{\infty}\right)$.

Proof, - Let $G_{1}$ be an open neighborhood of $G$ such that $\operatorname{cl}\left(G_{1}\right) \subset G$. Then $i_{A_{1}}(f, G)=i_{A_{1}}\left(f, G_{1}\right)$ and $i_{A_{\infty}}\left(f, G \cap A_{\infty}\right)=i_{A_{\infty}}\left(f, G_{2} \cap A_{\infty}\right)$. Lemmas 5 and 6, however, imply $i_{A_{1}}\left(f, G_{1}\right)=i_{A_{\infty}}\left(f, G_{1} \cap A_{\infty}\right)$.

Using Theorem 4 we can obtain a result which will prove more useful to us.

Theorem 5. - Let $A=\bigcup_{i=1}^{m} C_{i}$ be a finite union of oompact, convex sets $C_{i}$ in a $B$-space. Let $B=\bigcup_{i=1}^{m} D_{i}$ be a union of compact, convex sets $D_{i}$ such that $C_{i} \supset D_{i}$. Let 0 be an open subset of $A$ and $f: \operatorname{cl}(0) \rightarrow A$ a continuous map which has no fixed points on cl(0)-0. Assume that $f: 0 \cap B \rightarrow B$, Finally, let $K_{1}=\overline{\mathrm{co}} f(0), K_{n}=\overline{\mathrm{co}} f\left(0 \cap K_{n-1}\right), n>1$, and $K_{\infty}=\bigcap_{n \geq 1} K_{n}$. Assume that $B \supset K_{\infty} \cap A$. Then we have $i_{A}(f, 0)=i_{B}(f, 0 \cap B)$.

Proof. - Let us set $K_{n}^{\prime}=K_{n} \cap A$. We want to apply Property 5 to the $K_{n}^{\prime}$ in order to show that $i_{A}(f, 0)=i_{K_{\infty}^{\prime}}^{\prime}\left(f, 0 \cap K_{\infty}^{\prime}\right)$, where $K_{\infty}^{\prime}=\bigcap_{n \geq 1} K_{n}^{\prime}=K_{\infty} \cap A$. First notice that $f: 0 \cap K_{n}^{\prime} \rightarrow K_{n+1}^{\prime}, n>0$, for we have $f\left(0 \cap K_{0}^{\prime}\right)=f(0) \subset K_{1} \cap$ $\cap A=K_{1}^{\prime}$ and, generally, $f\left(0 \cap K_{n}^{\prime}\right)=f\left(0 \cap K_{n}\right) \subset \overline{\operatorname{co}} f\left(0 \cap K_{n}\right) \cap A=K_{n+1}^{\prime}$. Next observe that $K_{n} \supset K_{n+1}$. The proof is by induction: $K_{1}=\overline{\mathrm{co}} f(0) \supset \overline{\mathrm{co}} f\left(0 \cap K_{1}\right)$ $=K_{2}$, and if $K_{n} \supset K_{n+1}, K_{n+1}=\overline{\operatorname{co}} f\left(0 \bigcap K_{n}\right) \supset \overline{\operatorname{co}} f\left(0 \cap K_{n+1}\right)=K_{n+2}$. Thus we have that $K_{n}^{\prime}=\bigcup_{i=1}^{m}\left(K_{n} \cap C_{i}\right)$ and $\left(K_{n} \cap C_{i}\right) \supset\left(K_{n+1} \cap C_{i}\right)$. By Property 5, $i_{A}(f, 0)=i_{K_{\infty}^{\prime}}^{\prime}\left(f, 0 \cap K_{\infty}^{\prime}\right)=i_{K_{\infty} \cap A}\left(f, 0 \cap K_{\infty}\right)$.

Now suppose $B$ is as above. Consider the decreasing suquence of ANR's, $B_{0}=B, B_{n}=B \cap K_{n}, n \geq 1$. Since $B \supset K_{\infty} \cap A$, it is easy to see that $\bigcap_{n \geq 1} B_{n}=K_{\infty} \cap A \equiv K_{\infty}^{\prime}$. Since $f: 0 \cap K_{n} \rightarrow K_{n+1}, f: 0 \cap B_{n} \rightarrow B_{n+1}$. Finally, $B_{n}=\bigcup_{i=1}^{n}\left(D_{i} \cap K_{n}\right)$, and $D_{i} \cap K_{n} \supset D_{i} \cap K_{n+1}$. By Property $5, i_{B}(f, 0 \cap B)=$ $=i_{K_{\infty}^{\prime}}\left(f, 0 \cap K_{\infty}^{\prime}\right)$.

Q.E.D.

We now have those refinements of the classical fixed point index which we shall need, and we can proceed to more significant generalizations.

\section{D. - The fixed point index for functions like $k$-set-contractions.}

In this section we shall define a fixed point index for certains functions which behave like $k$-set-contractions, $k<1$. Our primary goal is to lay the groundwork for defining the fixed point index for $k$-set-contractions, $k<1$, and then for «nice»1-set-contractions (including condensing maps). However, some of our results here may have independent interest. It will not be hard to see that the elass of functions we shall consider includes functions which may not be $k$-set-contractions, $k<1$, with respect to any equivalent norm; and in fact such maps actually axise in applications. (See [30]). Furthermore, 
the functions we study in this section may provide greater flexibility in applications. Thus one can prove that the function $f$ considered in Corollary 3 of Section $A$ is a $k$-set contraction, $k<1$, with respect to some equivalent norm on the BarACH space, but the proof of this fact is much more difficult than the proof of Corollary 3 itself. However, the proof of Corollary 3 shows simply that $f$ lies in the class we consider, so the results of this section apply to it without proving it is a $k$-set-contraction, $k<1$, with respect to an eqnivalent norm.

Let us bogin with a general construction. Let $A$ be a subset of a BANACH space $B$ and let $g: A \rightarrow B$ be a continuous map. Let $K_{1}=\overline{c o} g(A)$, where co denotes the convex closure of a set. Let $K_{n}=\overrightarrow{\operatorname{co}} g\left(A \cap K_{n-1}\right), n>1$, and let $K_{\infty}=\bigcap_{n \geq 1} K_{n}$. We first claim that $K_{n} \supset K_{n+1}$. This follows because $K_{1}=\overline{\mathrm{co}}$ $g(A) \supset K_{2}=\overline{\operatorname{cog}}\left(A \cap K_{1}\right)$ and if $K_{n-1} \supset K_{n}, K_{n}=\overline{\operatorname{cog}}\left(A \cap K_{n-1}\right) \supset \overline{\operatorname{co}} g\left(A \cap K_{n}\right)=K_{n+1}$. Next notice that $g: A \cap K_{\infty} \rightarrow K_{\infty}$, for we have $g\left(A \cap K_{n}\right) \subset \operatorname{cog}\left(A \cap K_{n}\right)=K_{n+1}$ and $K_{n} \supset K_{n+1}$. Finally, of course, we see that $K_{\infty}$ is a closed, convex set.

The above construction will be used repeatedly, so we adopt some nota. tion concerning it. We write $K_{n}=K_{n}(g, A)$ and $K_{\infty}=K_{\infty}(g, A)$. If the set $A$ and the funclion $g$ are obvious, we may simply write $K_{n}$ and $K_{\infty}$ in the sequel.

Before proceeding further let us define the class of metric ANR's $X$ we shall study. Let $X$ be a closed subset of a $B_{A N A C H}$ space $B$ and assume the norm on $B$ induces the metric on $X$. Suppose there exists a locally finite cover $\left\{C_{i} \mid i \in I\right\}$ of $X$ by closed, convex sets $C_{i} \subset X$. More explicitly, suppose we have closed, convex sets $C_{i} \subset B, i \in I$, such that $X=\bigcup_{i \in I} C_{i}$, and such that for each $x \in X$ there is an open neighborhood $0_{x}$ of $x$ such that $0_{x} \cap C_{i}$ is empty except for finitely many $i \in I$. If $X$ is as above, we shall write $X \in \mathcal{F}$. Theorems of Hanner and Palais $[18,34]$ imply that if $X \in \mathscr{F}, X$ is a metric ANR. Notice that if $X \in \mathscr{F}$ is a metric ANR contained in a BANACH space $B$ and if $K \subset B$ is a compact, convex set, then $X \cap K$ is a finite union of com. pact, convex sets. For suppose $X=\bigcup_{i \in I} C_{i}, C_{i}$ a closed, convex set in $B$. For each $x \in K$, we can find an open neighborhood $0_{x}$ such that $0_{x} \cap C_{i}=\emptyset$ except for finitely many $i \in I$. Since $K$ is compact, we can cover $K$ by a finite number of these open sets, $0_{x_{1}}, \ldots, 0_{x_{n}}$. Then we have $K \cap C_{i}=\varnothing$ unless $i \in F, F=\left\{i \in I \mid C_{i} \cap 0_{x_{j}} \neq \emptyset\right.$ for some $\left.j, 1 \leq j \leq n\right\}$, a finite subset of 1 , and $X \cap K=\bigcup_{i \in F}\left(K \cap C_{i}\right)$.

Having established notation, let us derive a simple consequence of Theorem 5, Section $C$.

Lemma 1. - Let $G$ be an open subset of a metric ANR $X \in \mathfrak{F}, X \subset B, B$ a BANACH space: Let $g: \operatorname{cl}(G) \rightarrow \bar{X}$ be a continuous map without fixed points on $\operatorname{cl}(g)-G$ and assume that $K_{\infty}(g, G)$ is compact. Let $K$ be any compact, convex set in $B$ such that $K \supset K_{\infty}$ and $g: G \cap K \rightarrow K$. Let us write $K_{\infty}^{*}=K_{\infty} \cap X$ and $K^{*}=K \cap X$. Then we have $i_{K^{*}}\left(g, G \cap K^{*}\right)=i_{K_{\infty}^{*}}\left(g, G \cap K_{\infty}^{*}\right)$. (Notice that this makes sense since $G \cap K$ is an open subset of $K^{*}, G \cap K_{\infty}^{*}$ 
is an open subset of $K_{\infty}^{*}$, and $K^{*}$ and $K_{\infty}^{*}$ are compact, metric ANR's, since they are finite unions of compact, convex sets).

Proof. - By our comment above, $K^{*}=\bigcup_{i \in F}\left(K \cap C_{i}\right)$, where $F$ is a finite subset of $I, \quad X=\bigcup_{i \in I} C$, and notation is as above. Then we have $K_{\infty}^{*}=\bigcup_{i \in F}\left(K_{\infty} \cap C_{i}\right)$. It is elear that $K_{\infty}=K_{\infty}(g, G) \supset K_{\infty}\left(g, G \cap K^{*}\right)$, so that Theorem 5, Section $C$, implies $i_{K^{*}}\left(g, G \cap K^{*}\right)=i_{K_{\infty}^{*}}\left(g, G \cap K_{\infty}^{*}\right)$. Q.E.D.

Now let $G$ be an open subset of a space $X \in \mathfrak{F}$ and $g: \operatorname{cl}(G) \rightarrow X$ a con. tinuous function. Assume $g(x) \neq x$ for $x \in \mathrm{el}(G)-G$ and suppose that $K_{\infty}(g, G)$ is compact. Let us write $K_{\infty}^{*}=X \cap K_{\infty}(g, G)$ and define the generalized fixed point index of $g, i_{X}(g, G)=i_{K_{\infty}^{*}}\left(g, G \cap K_{\infty}^{*}\right)$. Notice that $K_{\infty}^{*}$ is a finite union of compact, convex sets, hence a compact, metric ANR. Also observe that $g(x) \neq x$ for $x \in \operatorname{cl}\left(G \cap K_{\infty}^{*}\right) \sim G \cap K_{\infty}^{*}$, since this set is contained in $(\operatorname{cl}(G) \sim G) \cap K_{\infty}^{*}$. Thus we see that $i_{K_{\infty}^{*}}^{*}\left(g, G \cap K_{\infty}^{*}\right)$ makes sense.

There is still one problem with our notation, however. If $X$ is a finite union of compact, convex sets, the fixed point index $i_{x}(g, G)$ is already defined. We must show that $\left.i_{X}(g, G)=i_{K_{\infty}^{*}} \mid g, G \bigcap K_{\infty}^{*}\right)$ in that case. Let $K=\overline{\mathrm{co}} X$, however, so $K^{*}=K \cap X=X$ and apply Lemma 1 ; we obtain $i_{X}(g, G)=$ $=i_{K^{*}}\left(g, G \cap K^{*}\right)=i_{K_{\infty}^{*}}\left(g, G \cap K_{\infty}^{*}\right)$. Thus our notation is permissible.

We want to show that the generalized fixed point index satisfies properties like those of the ordinary fixed point index.

Theorem 1. - Let $G$ be an open open subset of a space $X \in \mathscr{F}$ and $g$ a continuous function, $g: \operatorname{cl}(G) \rightarrow X$. Assume that $g(x) \neq x$ for $x \in \operatorname{cl}(G)-G$ and that $K_{\infty}(g, G)$ is compact. Let $S=\{x \in G \mid g(x)=x\}$ and assume that $S \subset G_{1} \cup G_{2}$ where $G_{1}$ and $G_{2}$ are two disjoint open sets included in $G$. Then $i_{X}\left(g, G_{i}\right)$ is defined, $i=1,2$ and $i_{X}\left(g, G_{1}\right)+i_{X}\left(g, G_{2}\right)=i_{X}(g, G)$.

Proof. - Notice that $K_{\infty}\left(g, G_{i}\right) \subset K_{\infty}(g, G)$ so $K_{\infty}\left(g, G_{i}\right)$ is compact and $i_{x}\left(g, G_{i}\right)$ is defined. For notational convenience let us write $K_{\infty}^{*}=X \cap K_{\infty}(g, G)$ and $K_{\infty, i}^{*}=X \cap K_{\infty}\left(g, G_{i}\right)$. By the ordinary additivity property, we have $i_{x}(g, G)=i_{K_{\infty}^{*}}\left(g, G \cap K_{\infty}^{*}\right)=i_{K_{\infty}^{*}}\left(g, G_{1} \cap K_{\infty}^{*}\right)+i_{K_{\infty}^{*}}\left(g, G_{2} \cap K_{\infty}^{*}\right)$. However, by Lem. ma 1 (where we take the set $K$ to be $K_{\infty}(g, G)$ ), we obtain $i_{K_{\infty}^{*}}^{*}\left(g, G_{j} \cap K_{\infty}^{*}\right)=$ $=i_{K_{\infty}^{*}, j}\left(g, G_{j} \cap K_{\infty}^{*}, j\right)=i_{X}\left(g, G_{j}\right)$. Thus we are done. Q.E.D.

Now suppose $g: G \rightarrow X$ is a continuous function, where $G$ is an open subset of a space $X \in \mathcal{F}$. Notice that we are not assuming $g$ is defined on $\operatorname{cl}(G)$. Assume that $S=\{x \in G \mid g(x)=x\}$ is compact and that there is an open neighborhood $V$ of $S$ such that $\operatorname{cl}(\nabla) \subset G$ and $K_{\infty}(g, \nabla)$ is compact. Then we define $i_{X}(g, G)=i_{X}(g, G)$. Just as in Section $B$, by using the additivity property and the fact that $i_{X}(g, G)=0$ if $g$ has no fixed points in $G$ (this follows immediately from our definition), we see that this definition does not depend on the particular $V$ chosen and agrees with our previous definition when $g$ in defined on $\operatorname{cl}(G)$ and $K_{\infty}(g, G)$ is compact. 
Theorem 2. - Let $I=[0,1]$ and let $\Omega$ be an open subset of $X \times I$, $X \in \mathscr{F}$. Let $F: \Omega \rightarrow X$ be a continuous map and assume $S=\{(x, t) \in \Omega: F(x, t)=x\}$ is compact. Suppose there exists an open subset 0 of $\Omega$ such that $S \subset 0$ and such that if we set $K_{1}(F, 0)=\overline{\operatorname{co}} F(0), K_{n}(F, 0)=\overline{\mathrm{co}} F\left(0 \bigcap\left(K_{n-1} \times I\right)\right), n>1$, and $K_{\infty}(F, 0)=\bigcap_{n \geq 1} K_{n}(F, 0)$, then $K_{\infty}(F, 0)$ is compact. Then if we set $\Omega_{t}=\{x:(x, t) \in \Omega\}$ and $F_{t}=F(\cdot, t), i_{X}\left(F_{t}, \Omega_{t}\right)$ is defined for $t \in I$ and $i_{X}\left(F_{0}, \Omega_{0}\right)=i_{X}\left(F_{1}, \Omega_{1}\right)$.

Proof. - For convenience write $K_{\infty}=K_{\infty}(F, 0)$ and $K_{\infty}\left(F_{t}, 0_{t}\right)=K_{\infty, t}$, where $0_{t}=\{x:(x, t) \in 0\}$. It is clear that $0_{t}$ is an open neighborhood of the fixed points of $F_{t}$ and $K_{x}\left(F_{t}, 0_{t}\right) \subset K_{\infty}(F, 0)$, a compact set. Thus $i_{X}\left(F_{t}, \Omega_{t}\right)$ is defined. As usual, write $K_{\infty}^{*}=K_{\infty} \cap X$ and $K_{\infty, t}^{*}=K_{\infty, t} \cap X$.

Next observe that $K_{n}=K_{n}(F, 0) \supset K_{n+1}$, for $K_{1} \supset K_{2}$ and if $K_{n} \supset K_{n+1}$, $K_{n+1}=\operatorname{co} F\left(0 \cap\left(K_{n} \times I\right)\right) \supset \operatorname{coF} F\left(0 \cap\left(K_{n+1} \times I\right)\right)=K_{n+2}$. It is also clear that $F: 0 \bigcap\left(K_{\infty} \times I\right) \rightarrow K_{\infty}$. For by our construction $F: 0 \bigcap\left(K_{n} \times I\right) \rightarrow K_{n+1}$, so that $F: 0 \cap\left(K_{\infty} \times I\right) \rightarrow \bigcap_{n \geq 2} K_{n}=K_{x}$. Consequently $F$ takes $0 \cap\left(K_{\infty}^{*} \times I\right)$ to $K_{\infty}^{*}$. Considering $F$ as a homotopy on the open set $0 \bigcap\left(K_{\infty}^{*} \times I\right)$ in $K_{a}^{*} \times I$, it is clear that the conditions of Theorem 2, Section $C$, are met, so that $i_{K_{\infty}^{*}}\left(F_{0}, 0_{0} \bigcap K_{\infty}^{*}\right)=i_{K_{\infty}^{*}}\left(F_{1}, 0_{1} \bigcap K_{\infty}^{*}\right)$. However, $K_{\infty} \supset K_{\infty, \imath}$, so that by Lemma 1 we have $i_{K_{\infty}^{*}}\left(F_{t}, 0_{t} \cap K_{\infty}^{*}\right)=i_{K_{\infty}^{*},}\left(F_{t}, 0_{t} \cap K_{\infty, \ell}^{*}\right)=i_{X}\left(F_{t}, \Omega\right)$. Combining these results, we see that $i_{X}\left(F_{0}, \Omega_{0}\right)=i_{X}\left(F_{1}, \Omega_{1}\right)$.

Corollary 1. - Let $\Omega$ be an open subset of $X \times I, X \in \mathscr{F}, I=[0,1]$. Let $f: \operatorname{cl}(\Omega) \rightarrow X$ be a continuons map such that $F(x, t) \neq x$ for $(x, t) \in \operatorname{cl}(\Omega) \sim \Omega$. In the notation of Theorem 2 , assume $K_{\infty}(F, \Omega)$ is compact. Then $i_{X}\left(F_{0}, \Omega_{0}\right)=i_{X}\left(F_{1}, \Omega_{1}\right)$.

Proof. - The open set 0 of Theorem 2 is provided by $\Omega$ itself. Thus it suffices to show $S=\{(x, t)=x\}$ is compact. Since $F(x, t) \neq x$ for $x \in \operatorname{cl}(\Omega) \sim \Omega$, $S=\{(x, t)=x\}$, and $S$ is closed. Thus we only have to show $S$ is contained in a compact set. However, if $F(x, t)=x$, it is easy to see that $x \in K_{\infty}(F, \Omega)$, a compact set.

Q.E.D.

Before proceding to our next theorem, let us prove some facts about a general construction.

Lemma 2. - Let $G_{1}$ and $G_{2}$ be subsets in BANAcH spaces $B_{1}$ and $B_{2}$ respectively. Let $g_{1}: G_{1} \rightarrow B_{2}$ and $g_{2}: G_{2} \rightarrow B_{1}$ be continuous maps. Let $K_{1}=$ co $g_{1}\left(G_{1}\right), K_{2}=\overline{\operatorname{co}} g_{2}\left(G_{2} \cap K_{1}\right)$ and generally let $K_{2 n+1}=\overline{\operatorname{co}} g_{1}\left(G_{1} \cap K_{2 n}\right)$ and $K_{2 n+2}=$ $=\overline{\mathrm{co}} g_{2}\left(G_{2} \cap K_{2 n+1}\right)$. Let $K_{\text {odd }}=\bigcap_{n=1}^{\infty} K_{2 n-1}$ and $K_{\text {even }}=\bigcap_{n=1}^{\infty} K_{2 n}$. Let $H_{1}$ be any subset of $g_{1}^{-1}\left(G_{2}\right)$ and let $H_{2}$ be any subset of $g_{2}^{-1}\left(G_{1}\right)$. Then we have

(1) $K_{\text {even }} \supset K_{\infty}\left(g_{2} g_{1}, H_{1}\right), K_{\text {odd }} \supset K_{\infty}\left(g_{1} g_{2}, H_{2}\right)$.

(2) $g_{2} g_{1}: H_{1} \cap K_{\text {even }} \rightarrow K_{\text {even }}, g_{1} g_{2}: H_{2} \cap K_{\text {odd }} \rightarrow K_{\text {odd }}$.

(3) $g_{1}: G_{1} \cap K_{\text {even }} \rightarrow K_{\text {odd }}, g_{2}: G_{2} \bigcap K_{\text {odd }} \rightarrow K_{\text {even }}$. 
Proof. - To prove the first assertion, it suffices to show that $K_{n}\left(g_{1} g_{2}, H_{2}\right) \subset$ $\subset K_{2 n-1}$ and $K_{n}\left(g_{2} g_{1}, H_{1}\right) \subset K_{2 n}$. The proof is by induction. First consider the case $n=1$. We have $K_{1}\left(g_{1} g_{2}, H_{2}\right)=\overline{\mathrm{co}} g_{1}\left(g_{2}\left(H_{2}\right)\right) \subset \overline{\mathrm{co}} g_{1}\left(G_{1}\right)=K_{1}$. Similarly, using that $g_{1}\left(H_{1}\right) \subset G_{2}$, we obtain that $K_{1}\left(g_{2} g_{1}, H_{1}\right) \subset \overline{\operatorname{co}} g_{2}\left(\overline{\operatorname{co}} g_{1}\left(H_{1}\right) \cap G_{2}\right) \subset$ $\subset \overrightarrow{\mathrm{co}} g_{2}\left(\overline{\mathrm{co}} g_{1}\left(G_{1}\right) \cap G_{2}\right)=K_{2}$. Generally, suppose we have shown that $K_{n}\left(g_{1} g_{2}, H_{2}\right)$ $\subset K_{2 n-1}$ and $K_{n}\left(g_{2} g_{1}, H_{1}\right) \subset K_{2 n}$. Then we have that $K_{n+1}\left(g_{1} g_{2}, H_{2}\right)=\overline{\operatorname{co}}\left(g_{1} g_{2}\right)\left(H_{2} \cap\right.$ $\left.\cap K_{n}\left(g_{1} g_{2}, H_{2}\right)\right) \subset \overline{\operatorname{co}}\left(g_{1} g_{2}\right)\left(H_{2} \cap K_{2 n-1}\right)=\overline{\operatorname{co}} g_{1}\left(g_{2}\left(H_{2} \cap K_{2 n-1}\right) \cap G_{1}\right) \subset \overline{\operatorname{co}} g_{1}\left(\overline{\operatorname{co}} g_{2}\left(G_{2} \cap\right.\right.$ $\left.\left.\cap K_{2 n-1}\right) \cap G_{1}\right)=\overline{\operatorname{cog}}\left(K_{2 n} \cap G_{1}\right)=K_{2 n+1}$. Similarly, we find that $K_{n+1}\left(g_{2} g_{1}, H_{1}\right)$ $=\overline{\mathrm{co}}\left(g_{2} g_{1}\right)\left(H_{1} \cap K_{n}\left(g_{2} g_{1}, H_{1}\right)\right) \subset \overline{\mathrm{co}}\left(g_{2} g_{1}\right)\left(H_{1} \cap K_{2 n}\right)=\overline{\operatorname{co}} g_{2}\left(g_{1}\left(H_{1} \cap K_{2 n}\right) \cap G_{2}\right) \subset \overline{\mathrm{co}} g_{2}$ $\left.\overline{(\operatorname{co}} g_{1}\left(G_{2}\right)\right)=K_{2 n+2}$. This completes the inductive step and proves our first assertion.

We have shown above that $K_{2 n-1} \supset K_{2 n+1}$ and $K_{2 n} \supset K_{2_{n+2}}$. To prove our final claim it thus suffices to show that $g_{1}: G_{1} \cap K_{2 n} \rightarrow K_{2_{n+1}}$ and $g_{2}: G_{2} \cap$ $\cap K_{2 n-1} \rightarrow K_{2 n}$. However we have that $g_{1}\left(G_{1} \cap K_{2 n}\right) \subset \overline{\operatorname{co}} g_{1}\left(G_{1} \cap K_{2 n}\right)=K_{2 n+1}$ and $g_{2}\left(G_{2} \cap K_{2 n-1}\right) \subset \overline{\operatorname{co}} g_{2}\left(G_{2} \cap K_{2 n-1}\right)=K_{2 n}$, so we are done. Q.E.D.

Theorem 3. - Let $G_{1}$ and $G_{2}$ be open sets in spaces $X_{1}$ and $X_{2}$ respectively, $X_{i} \in \mathscr{F F}, i=1$, 2. Let $g_{1}: G_{1} \rightarrow X_{2}$ and $g_{2}: G_{2} \rightarrow X_{1}$ be continuous maps. Assume $S=\left\{x \in g_{1}^{-1}\left(G_{2}\right)\left\{\left(g_{2} g_{1}\right)(x)=x\right\}\right.$ is compact. Finally, assume that $K_{\text {odd }}$ and $K_{\text {eren }}$ are compact, where the notation is the same as in Lemma 2. Then we have that $i_{X_{1}}\left(g_{2} g_{1}, g_{1}^{-1}\left(G_{2}\right)\right)$ and $i_{X_{2}}\left(g_{1} g_{2}, g_{1}^{-1}\left(G_{1}\right)\right)$ are defined and equal.

Proof. - Let $S=\left\{x \in g_{2}^{-1}\left(G_{1}\right) \mid\left(g_{1} g_{2}\right)(x)=x\right\}$. We know that $S_{i}$ is compact, $i=1,2$, so let $H_{i}$ be an open neighborhood of $S_{i}$ such that $\operatorname{el}\left(H_{1}\right) \subset g_{1}^{-1}\left(G_{2}\right)$ and $\operatorname{cl}\left(H_{2}\right) \subset g_{2}^{-1}\left(G_{1}\right)$. By Lemma 2, we know that $K_{x}\left(g_{2} g_{1}, H_{1}\right) \subset K_{\text {eren }}$ and $K_{\infty}\left(g_{1} g_{2}, H_{2}\right) \subset K_{\text {odd }}$, so that both these sets are compact and $i_{X_{1}}\left(g_{2} g_{1}, H_{1}\right)$ and $i_{X_{2}}\left(g_{1} g_{2}, H_{2}\right)$ are defined.

We also showed in Lemma 2 that $g_{2} g_{1}: H_{1} \cap K_{\text {even }} \rightarrow K_{\text {even }}$ and $g_{1} g_{2}: H_{2} \cap$ $\cap K_{\text {odd }} \rightarrow K_{\text {odd }}$. By Lemma 1 of this section, it follows that $i_{X_{1}}\left(g_{2} g_{1}, H_{1}\right)=$ $=i_{K_{\text {eren }}^{*}}\left(g_{2} g_{1}, H_{1} \cap K_{\text {even }}^{*}\right)$ and $i_{X_{2}}\left(g_{1} g_{2}, H_{2}\right)=i_{K_{\text {odd }}^{*}}\left(g_{1} g_{2}, H_{2} \cap K_{\text {odd }}^{*}\right)$, where we have written $K_{\text {odd }}^{*} \equiv K_{\text {odd }} \cap X_{2}$ and $K_{\text {even }}^{\text {s. }} \equiv K_{\text {even }} \cap X_{1}$. Thus to complete the proof of Theorem 3 it suffices to show that $i_{K_{\text {even }}^{*}}\left(g_{2} g_{1}, H_{1} \cap K_{\text {even }}^{*}\right)=i_{K_{\text {odd }}^{*}}\left(g_{1} g_{2}\right.$, $H_{2} \cap K_{\text {odd }}^{*}$. Bu we showed in Lemma 2 that $g_{1}: G_{1} \cap K_{\text {even }} \rightarrow K_{\text {odd }}$ and $g_{2}: G_{2} \cap K_{\text {odd }} \rightarrow K_{\text {even }}$, so it follows that $g_{1}: G_{1} \cap K_{\text {even }}^{*} \rightarrow K_{\text {odd }}^{*}$ and $g_{2}: G_{2} \cap$ $\cap K_{\text {odd }}^{*} \rightarrow K_{\text {even }}^{*}$. By the commutativity property for the ordinary fixed point index, and the fact that all fixed points of $g_{2} g_{1}$ in $H_{1} \cap K_{\text {even }}^{*}\left(=S_{1}\right)$ lie in $g_{1}^{-1}\left(G_{2} \cap K_{\mathrm{dd}}^{*}\right)$ and similarly for $g_{1} g_{2}$, we obtain $i_{K_{\mathrm{even}}^{*}}\left(g_{2} g_{1}, g_{1}^{-1}\left(G_{2} \cap K_{\text {odd }}^{*}\right)\right)=$ $=i_{K_{\text {even }}^{*}}\left(g_{2} g_{1}, H_{1} \cap K_{\text {even }}^{*}\right)=i_{K_{\text {odd }}^{*}}\left(g_{1} g_{2}, g_{2}^{-1}\left(g_{1} \cap K_{\text {even }}^{*}\right)\right)=i_{K_{\text {odd }}^{*}}\left(g_{1} g_{2}, H_{2} \cap K_{\text {odd }}^{*}\right)$. This completes the proof of our theorem.

Q.E.D.

Let us now take stock of our progress. In Theorems 1, 2, and 3 we have proved, respectively, generalizations of the additivity property, the homotopy property, and the commutativity property. We have not generalized the normalization property here, but we shall do so in the next section. 
We have also shown that our generalized fixed point index agrees with the classical fixed point index for compact metric ANR's, at least when both are defined. It is natural to ask if our fixed point index agrees with the the Leray-Schadder fixed point index, or equivalently with the appropriate Leray-Schauder degree. Let $G$ be an open subset of a Barach space $X$ and $g: \operatorname{cl}(G) \rightarrow X$ a continuous map such that $g(x) \neq x$ for $x \in \partial G$. Assume that $g$ is compact, i.e., $g(G)$ has compact closure. Leray and Schadder then defined a fixed point index for $g$ (and consequently a degree for $I-g, I=$ the identity function). We shall denote this degree by $\operatorname{deg}_{L S}(I-g, G, 0)$. On the other hand, $K_{\infty}(g, G)$ is certainly compact, so $i_{X}(g, G)$ is defined.

Proposimiox 1. $i_{X}(g, G)=\operatorname{deg}_{L S}(I-g, G, 0)$.

Proof. - $\operatorname{deg}_{L S}(I-g, G, 0)$ can be defined to be $i_{A}(g, G \cap A)$, where $A$ is any compact, metric ANR in $X$ containing $g(G)$. (Other definitions of the Leray-Schatder degree are possible. We shall not prove the equivalence of these definitions here, though it is not hard to do so). In particular, we can take $A=\overline{\operatorname{co}} g(G)$. But then $A \supset K_{\infty}(g, G), A$ is compact and convex, and $g: G \cap A \rightarrow A$. Thus we are in the situation of Lemma 1 , and $i_{A}(g, G \cap A)=$ $=i_{K_{\infty}(g, G)}\left(g, G \cap K_{\infty}(g, G)\right)=i_{X}(g, G)$.

Q.E.D.

Let us apply the above result to obtain a proposition of some indepen. dent interest. Proposition 2 is used without proof in the Browder-Nussbuav article [10] in order to identify two different ways of defining a generalized degree. Needless to say, it can be proved directly without the elaborate ap. paratus assembled here.

Proposition 2. - Let $G$ be an open subset of a Banach space $X_{1}$. Let $C: \operatorname{cl}(G) \rightarrow X_{2}$ be a compact map into a $\mathrm{B}_{\mathrm{ANACH}}$ space $X_{2}$. Let $h: \operatorname{cl}(G) \rightarrow X_{2}$ be a homeomorphism such that $h(\mathrm{cl}(G))$ is closed and $h(G)$ is open. Assume that $h(x)-C(x) \neq 0$ for $x \in \operatorname{cl}(G)-G$. Then we have $\operatorname{deg}_{L S}\left(I-C h^{-1}, h G, 0\right)$ and $\operatorname{deg}_{L S}\left(I-h^{-1} C, C^{-1}(h G), 0\right)$ are defined and equal.

Proof. - Let $G_{1}=G$ and $G_{2}=h G$ and recall that we are assuming $G_{2}$ to be open. By Proposition 1 it suffices to show that $i_{X_{2}}\left(C h^{-1}, G_{2}\right)$ and $i_{X_{1}}\left(h^{-1} C, C^{-1}\left(G_{2}\right)\right)$ are defined and equal. However, notice that we are in the situation of the commutativity property (Theorem 3): $h^{-1}: G_{2} \rightarrow G_{1} \subset \bar{X}_{1}$ and $C: G_{1} \rightarrow X_{2}$. Thus it suffices to show that the hypotheses of Theorem 3 are met. Since $C$ is compact, it is clear that $K_{\text {odd }}$ and $K_{\text {even }}$ are compact. We have to show that $S_{1}=\left\{x \in\left(h^{-1}\right)^{-1}\left(G_{1}\right) \mid\left(C h^{-1}\right)(x)=x\right\}=\left\{x \in G_{2} \mid\left(C h^{-1}\right)(x)=x\right\}$ is compact. However, $h(\operatorname{cl} G)$ is a closed set by assumption, and $C h^{-1}$ is a compact map defined on $h(\operatorname{cl} G)$, so $\tilde{S}_{1}=\left\{x \in h(\mathrm{cl} G) \mid\left(C h^{-1}\right)(x)=x\right\}$ is compact. But $S_{1}=\tilde{S}_{1}$, for if $\left(C h^{-1}\right)(x)=x$ and $x \notin S_{1}$, then $x \in h(\mathrm{cl} G)-h(G)=h(\partial G), x=$ 
$=h(y), y \in \mathfrak{J} G$, and $h y-C y=0$, a contradiction. Thus the hypotheses of Theorem 3 are satisfied, and we have $i_{X_{2}}\left(C h^{-1}, h G\right)=i_{X_{1}}\left(h^{-1} C, C^{-1}(h G)\right)$.

\section{E. - The fixed point index for local strict-set-contractions.}

In this section we shall show that our previous results can be applied in a neat way to give a fixed point index for local strict-set-contractions. We begin with a lemma.

Lemma 1. - Let $G$ be an open subset of a space $X \in \mathcal{F}$ and $f: G \rightarrow X$ a local strict-set-contraction such that $S=\{x \in G \mid f(x)=x\}$ is compact. Then there exists an open neighborhood $V$ of $S$ such that $K_{\infty}(f, V)$ is compact. If $G$ is bounded and $f: \operatorname{cl}(G) \rightarrow X$ is a $k$-set-contraction, $k<1$, such that $g(x) \neq x$ for $x \in \partial G$, then $S$ is necessarily compact.

Proof. - If $f$ is a local strict-set-contraction and we assume $S$ compact, then for each $x \in S$, there is a bounded open neighborhood $N_{x}$ such that $f \mid N_{x}$ is a $k_{x}$-set-contraction, $k_{x}<1$. Since $S$ is compact there exists a finite open covering of $S$, say $N_{x_{i}}, N_{x_{2}}, \ldots, N_{x_{n}}$. For convenience set $k_{x_{i}}=k_{i}$ and $N_{x_{i}}=N_{i}, 1 \leq i \leq n$. Let $V=\bigcup_{i=1}^{n} N_{i}$. To show that $K_{\infty}(f, V)$ is compact, it suffices to show that $f \mid V$ is a $k$-set-contraction for some $k<1$. Let $k=\max$ $\left\{k_{i}: 1 \leq i \leq n\right\}$ and let $A$ be any subset of $V$. We have $f(A)=\bigcup_{i=1}^{n} f\left(A \cap N_{i}\right)$, so that $\gamma(f(A))=\max \left\{\gamma\left(f\left(A \cap N_{i}\right)\right)\right\} \leq \max \left\{k \gamma\left(A \cap N_{i}\right)\right\} \leq k \gamma(A)$.

If $G$ is bounded and $f: \operatorname{cl}(G) \rightarrow X$ is $k$-set-contraction, $k<1$, such that $f(x) \neq x$ for $x \in \partial G, S=\{x \in \operatorname{cl}(G): f(x)=x\}$ so $S$ is closed. Furthermore, $S=f(S)$, so $\gamma(S) \leq k \gamma(S), k<1$, and we must have $\gamma(S)=0$. It follows that $S$ is compact.

Q.E.D.

Let $G$ be an open subset of a space $X \in \mathscr{F}$ and assume $f: G \rightarrow X$ is a local strict-set-contraction such that $S=\{x \in G \mid f(x)=x\}$ is compact. By Lemma 1, there exists an open neighborhood $V$ of $S$ such that $K_{\infty}(f, V)$ is compact. By the results of the previous section there is defined a generalized fixed point index $i_{X}(t, G) \equiv i_{X_{\cap} K_{x}(f, V)}\left(f, V \cap X \cap K_{\infty}(f, V)\right)$. We now examine how Theorems $1-3$ of Section $D$ translate to our context.

Theorem 1. - (The additive property). Let $G$ be an open subset of a space $X \in \mathscr{F}$ and $f: G \rightarrow X$ a local strict-set-contraction such that $S=\{x \in G$ : $f(x)=x\}$ is compact. Assume that $S \subset G_{1} \cup G_{2}$, where $G_{1}$ and $G_{2}$ are disjoint open subsets of $G$. Then $i_{x}(f, G)=i_{x}\left(f, G_{1}\right)+i_{x}\left(f, G_{2}\right)$.

Proof. - By Lemma 2 there exists an open neighborhood $V$ of $S$ such that $\operatorname{cl}(\nabla) \subset G$ and $K_{x}(f, V)$ is compact. By our definition $i_{X}(f, G)=i_{X}(f, V)$ and $i_{X}\left(f, G_{j}\right)=i_{X}\left(f, G_{j} \cap V\right), j=1,2$. By Theorem 1, Section $D, i_{X}(f, V)=$ $=i_{X}\left(t, G_{1} \cap \nabla\right)+i_{X}\left(t, G_{2} \cap \nabla\right)$.

Q.E.D. 
Theorem 2. - (The homotopy property). Let $I=[0,1]$ and let $\Omega$ be an open subset of $X \times 1, X \in \mathscr{F}$. Let $F: \Omega \rightarrow X$ be a continuous function and assume that $F$ is a local strict-set-contraction in the following sense: given $(x, t) \in \Omega$, there exists an open neighborhood of $(x, t)$ in $\Omega, N_{(x, t)}$, such that for any subset $A$ of $X, \gamma\left(F\left(N_{(x, t)} \cap(A \times I)\right) \leq k_{(x, t)} \gamma(A), k_{(x, t)}<1\right.$. Assume that $S=\{(x, l) \in \Omega: F(x, t)=x\}$ is compact. Then (in the notation of Theorem 2, Section $D) i_{X}\left(F_{t}, \Omega_{t}\right)$ is defined for $t \in I$ and $i_{X}\left(F_{0}, \Omega_{0}\right)=i_{X}\left(F_{1}, \Omega_{1}\right)$.

Proof. - By Theorem 2, Section $D$, (using the notation of that theorem) it suffices to find an open neighborhood 0 of $S, 0 \subset \Omega$, such that $K_{x}(F, 0)$ is compact. Because $F$ is a local strict-set-contraction and because $S$ is com. pact, there exist a finite open covering of $S$ by bounded open sets $N_{i}$ in $\Omega$, $1 \leq i \leq n$, such that for $A \subset X, \gamma\left(F\left(N_{i} \cap(A \times I)\right)\right) \leq k_{i} \gamma(A), k_{i}<1$. Set $0=\bigcup_{i=1}^{n} N_{i}$ and $k=\max \left\{k_{i}\right\}<1$. Just as in the proof of Lemma 1 , for $A \subset X$ we find $\gamma(F(0 \cap(A \times I))) \leq k \gamma(A)$.

Recall that $K_{1}(F, 0)=\overline{\mathrm{co}} F(0)$ and $K_{n+1}(F, 0)=\overline{\mathrm{co}} F\left(0 \cap\left(K_{n} \times I\right)\right)$, and for notational convenience set $K_{n}=K_{n}(F, 0)$ and $K_{\infty} \equiv K_{\infty}(F, 0)=\bigcap_{n \geq 1} K_{n}$. To prove our theorem it suffices to show $\gamma\left(K_{n}\right) \rightarrow 0$, since then $K_{\infty}$ will be com. pact. Since 0 is bounded, $F(0)$ is bounded and $\gamma\left(K_{1}\right)=M$ is defined. Generally $\gamma\left(K_{n+1}\right)=\gamma\left(\cos F\left(0 \cap\left(K_{n} \times I\right)\right)\right)=\gamma\left(F\left(0 \cap\left(K_{n} \times I\right)\right)\right) \leq k \gamma\left(K_{n}\right)$. This shows $\gamma\left(K_{n+1}\right) \leq$ $\leq k^{n} M \rightarrow 0$.

Q.E.D.

Corollary 1. - Let $I=[0,1]$ and let $\Omega$ be a bounded, open subset of $X \times I, \quad X \in \mathcal{F}$. Let $F: \operatorname{cl}(\Omega) \rightarrow X$ be a continuous function and assume $F(x, t) \neq x$ for $x \in \operatorname{cl}(\Omega) \sim \Omega$. Assume that $F$ is a $k$-set-contraction, $k<1$, in the following sense: for all bounded $A \subset X, \gamma(F(\Omega \cap(A \times I))) \leq k \gamma(A)$. Then $i_{X}\left(H_{t}, \Omega_{t}\right)$ is defined and $i_{X}\left(F_{0}, \Omega_{0}\right)=i_{X}\left(F_{1}, \Omega_{1}\right)$.

Proof. - By Theorem 2 it suffices to show $S=\{(x, t) \in \Omega: F(x, t)=x\}$ is compact. Since $F(x, t) \neq x$ for $x \in \operatorname{cl}(\Omega)-\Omega, S=\{(x, t) \in \operatorname{cl}(\Omega): F(x, t)=x\}$ and $S$ is closed. Let $T=\{x:(x, t) \in S$ for some $t \in I\}$. Clearly $(T \times I) \cap \Omega \supset S$ so that $F((T \times I) \cap \Omega) \supset F(S)=T$. Thus we have $\gamma(T) \leq \gamma(F((T \times I) \cap \Omega)) \leq k \gamma(T)$, so $\gamma(T)=0$ and $T$ has compact closure. It follows that $\mathrm{cl}(T) \times I$ is compact, and since $S \subset \mathrm{cl}(T) \times 1, S$ is compact.

Q.E.D.

In order actually to apply Theorem 2 we need some simple conditions under which a homotopy is permissible. We start with a general proposition.

Proposition 1. - Let $(M, P)$ be a compact metric space and let $A$ be a closed, bounded subset of a metric space $(X, d)$. Let $F: A \times M \rightarrow X$ be a continuous function. For any $m_{0} \in M$ assume that for every $\varepsilon>0$, there exists a $\delta>0$ such that $p\left(m, m_{0}\right)<\delta$ implies $d\left(F_{m}(x), F_{m_{0}}(x)\right)<\varepsilon$ for all $x \in A\left(F_{m}(x) \equiv F(x, m)\right)$. Eurther assume that for all $m \in M, F_{m}$ is a $k$-set-contraction, $k<1, k$ independent of $m$. Then if $B \subset A, \gamma(F(B \times M)) \leq k \gamma(B)$. Furthermore, $\{x \in A$ : $F(x, m)=x$ for some $m \in M\} \equiv S$ is compact or empty. 
Proof. - Suppose $B \subset A$ and $\gamma(B)=r$. We have to show that $F(B \times M)$ can be covered by a finite number of sets of diameter $\leq k r+\varepsilon$ for every $\varepsilon>0$. Select $\varepsilon>0$. For each $m \in M$ there is an open ball $B_{\delta(m)}(m)$ about $m$ such that if $\rho\left(m^{\prime}, m\right)<\delta(m), d\left(F_{m^{\prime}}(x), F_{m}(x)\right)<\varepsilon / 3$ for $x \in A$. Since $M$ is compact, cover $M$ by a finite number of ball $B_{\delta\left(m_{i} i\right.}\left(m_{i}\right), 1 \leq i \leq n$. Since $F_{m_{i}}$ is a $k$-setcontraction, there exists a covering of $F_{m_{i}}(B)$ by a finite number of sets $S_{i j}$, $1 \leq j \leq n_{i}$, of diameter $\leq k r+\varepsilon / 3$. We know that $\gamma\left(N_{\varepsilon\{3}\left(S_{i j}\right)\right) \leq k r+\varepsilon$. On the other hand, suppose that $x \in B$ and $m \in M$. Then for some $i$, $\left(m, m_{i}\right)<\delta\left(m_{i}\right)$, so that $d\left(F(x, m), F\left(x, m_{i}\right)\right)<\varepsilon / 3$. But $F\left(x, m_{i}\right) \in S_{i j}$ for $j, 1 \leq j \leq n_{i}$, so $F(x, m) \in N_{\varepsilon ; 3}\left(S_{i j}\right)$. This shows $F(B \times M) \subset \bigcup_{i, j} N_{\varepsilon j 3}\left(S_{i j}\right)$, so that $\gamma(F(B \times M)) \leq k r+\varepsilon$.

If $S=\{x \in A: F(x, m)=x$ for some $m \in M\}$, since $A$ is closed and $M$ compact, it is clear that $S$ is closed. Also, we see that $S \subset F(S \times M)$, so $\gamma(S) \leq \gamma(F(S \times M)) \leq k \gamma(S)$. It follows that $\gamma(S)=0$, so $S$ is compact.

Q.E.D.

Coroliary 2. - Let $A$ be a closed, bounded subset of a Banach space $X, f: A \rightarrow X$ a $k$-set-contraction, $k<1$. Let $I$ denote the identity map. Then $I-f$ is a proper map, i.e., $(I-f)^{-1}$ (compact set $)=$ compact set.

Proof. - Let $M$ be a compact subset of $X$. We want to show that $(I-f)^{-1}(M)$ is compact. Let $F(x, m)=f(x)+m, x \in A, m \in M$. Clearly the conditions of Proposition 1 are met, so $\{x: F(x)+m=x$ for some $m \in M\}=$ $=(I-f)^{-1}(M)$ is compact.

Q.E.D.

Recally that a proper map from one metric space to another is closed (that is, takes closed sets to closed sets), so that $I-f$ is closed.

Corollary 3. - Let $G$ be a bounded, open subset of a space $X, X \in F$ Let $J=[0,1]$ and let $F: o l(G) \times J \rightarrow X$ be a continuous map such that $F_{t}(x) \equiv F(x, t) \neq x$ for $x \in \operatorname{cl}(G)-G$. Assume that each $F_{t}$ is $k$-set-contraction, $k<1, k$ independent of $t$. Finally assume that for each $t_{0} \in J$ and $\varepsilon>0$, there exists a $\delta>0$ snch that for $\left|t-t_{0}\right|<\delta, t \in J$, and $x \in \operatorname{cl}(G), \| F(x, t)-$ $-F\left(x, t_{0}\right) \|<\varepsilon$. Then we have $i_{X}\left(F_{0}, G\right)=i_{X}\left(F_{1}, G\right)$.

Proof. - By Proposition 1, for $A \subset \operatorname{cl}(G), \gamma(F(A \times J)) \leq k \gamma(A)$, so all the concitions of the homotopy property are met.

Q.E.D.

Conollari 4. - Let $t_{0}$ and $t_{1}$ be, respectively, $k_{0}$ and $k_{1}$-set-contractions, $f_{i}: \operatorname{cl}(G) \rightarrow X$ where $G$ and $X$ are as in Corollary $2, k_{i}<1$. Let $F(x, t)=t f_{0}(x)+$ $+(1-t) f_{1}(x), t \in J=[0,1]$. Then $\gamma(F(A \times J)) \leq k \gamma(A), k=\max \left(k_{0}, k_{1}\right)$.

Proof. $-F_{t}=t t_{0}+(1-t) f_{1}$ is a $k$-set-contraction, so it suffices to show that $t \rightarrow F_{t}$ is continuous. $f_{0}[\operatorname{cl}(G)]$ and $f_{1}[\operatorname{cl}(G)]$ are bounded sets, say bounded by $M$. Thus we have $\left\|t t_{0}(x)+(1-t) f_{1}(x)-t_{0} t_{0}(x)-\left(1-t_{0}\right) f_{1}(x)\right\| \leq\left|t-t_{0}\right|$ $\left\|t_{0}(x)\right\|+\left|t-t_{0}\right|\left\|t_{1}(x)\right\| \leq 2\left|t-t_{0}\right| M$. Thus the conditions of Proposition 1 are met.

Q.E.D. 
Corollary 5. - Let $G$ be a bounded, open, convex set in a BanaCH space $X, f: \operatorname{cl}(G) \rightarrow X$ a $k$-set-contraction, $k<1$. Assume $f(\partial G) \subset \operatorname{cl}(G)$. Then $f$ has a fixed point in $\operatorname{cl}(G)$.

Proof. - Take $x_{0} \in G$ and consider the homotopy $t f+(1-t) x_{0}$. If $f(x) \neq x$ for $x \in \partial G, t f(x)+(1-t) x_{0} \neq x$, since $t f(x)+(1-t) x_{0} \in G$ for $t \neq 1$. Thus in this case the homotopy is permissible and $i_{X}(f, G)=i_{X}\left(x_{0}, G\right)$. One easily shows thbt $i_{X}\left(x_{0}, G\right)$ is given by the Lefschetz number of the identity map of a point to itself, so $i_{x}\left(x_{0}, G\right)=1$ and $t$ has a fixed point. If $f(x)=x$ for some $x \in \partial G$, of course we are done immediately.

Q.E.D.

It is clear that if $0 \in G$ any open set and $f(x)+s x$ for $s \geq 1$, and $x \in \partial G$, then the same proof shows $f$ has a fixed point.

Next, let us establish a commutativity property. The reader should note that in the proof of Theorem 3 below, we write $\gamma$ generically to denote measure of noncompactness in two different metrie spaces, $X_{1}$ and $X_{2}$. Of course the measures of noncompactness in these metric spaces actually are, in general, different.

Theorem 3. - (The commutativity property). Let $G_{1}$ and $G_{2}$ be open subset of spaces $X_{1}$ and $X_{2}$, respectively, $X_{i} \in F$. Let $g_{1}: G_{1} \rightarrow X_{2}$ be a $k_{1}$-setcontraction and $g_{2}: G_{2} \rightarrow X_{1}$ be a $k_{2}$-set-contraction. Assume that $S_{1}=\left\{x \in g_{1}^{-1}\right.$ $\left.\left(G_{2}\right) \mid\left(g_{2} g_{1}\right)(x)=x\right\}$ is compact. Finally assnme that $k_{1} k_{2}<1$. If $k_{1}=0$ we only need assume $g_{2}$ is continuous and defined on $\operatorname{cl}\left(G_{2}\right)$. Then we have $i_{X_{1}}\left(g_{2} g_{1}\right.$, $\left.g_{1}^{-1}\left(G_{2}\right)\right)=i_{X_{2}}\left(g_{1} g_{2}, g_{1}^{-1}\left(G_{1}\right)\right)$.

REMARK. - If $g_{1}^{-1}\left(G_{2}\right)$ is bounded, $\left(g_{2} g_{1}\right)(x) \neq x$ for $x \in \operatorname{cl}\left(g_{1}^{-1}\left(G_{2}\right)\right) \sim g_{1}^{-1}\left(G_{2}\right)$, and $k_{1} k_{2}<1$, then $S_{1}$ will be compact.

Proof. - First we show that we can assume $G_{1}$ and $G_{2}$ bounded and $g_{i}$ defined on $\operatorname{cl}\left(G_{i}\right)$. Since $S_{\mathrm{x}}$ is compact, by Lemma 1 , Section $C, S_{2}=\left\{x \in g_{2}^{-1}\right.$ $\left.\left(G_{1}\right):\left(g_{1} g_{2}\right)(x)=x\right\}$ is compact. Let $U_{i} \subset G_{i}$ be a bounded open neighborhood of $S_{i}$ with $\mathrm{cl}\left(U_{i}\right) \subset G_{i}$ and let $H_{1}=\left\{x \in U_{1}: g_{1}(x) \in U_{2}\right\}, H_{2}=\left\{x \in U_{2}: g_{2}(x) \in U_{1}\right\}$. By Lemma 1 , Section $C$, it is easy to see that $H_{i}$ is an open neighborhood of $S_{i}$.

Thus $i_{X_{1}}\left(g_{2} g_{1}, g_{1}^{-1}\left(G_{2}\right)\right)=i_{X_{1}}\left(g_{2} g_{1}, H_{1}\right)$ and similarly for $H_{2}$. Since $H_{1}=\left(g_{1}\right)$ $\left.U_{1}\right)^{-1}\left(U_{2}\right)$ and $H_{2}=\left(g_{2} \mid U_{2}\right)^{-1}\left(U_{1}\right)$, we see that (since we can restrict attention to $U_{1}$ and $U_{2}$ ) we may as well assume $G_{1}$ and $G_{2}$ bounded and $g_{i}$ defined on $\operatorname{cl}\left(G_{i}\right)$.

Thus assume $G_{1}$ and $G_{2}$ bounded and $g_{i}$ defined on $\operatorname{cl}\left(G_{i}\right)$. In the notation of Theorem 3, Section $C$, it suffices to show $K_{\text {odd }}$ and $K_{\text {even }}$ are compact. If $k_{1}=0$, we know that el $\left(g_{1}\left(G_{1}\right)\right)$ is compact, so $K_{1}=\overline{\operatorname{co}} g_{1}\left(G_{1}\right)$ is compact. Also, we have $K_{2}=\overline{\operatorname{co}} g_{2}\left(G_{2} \cap K_{1}\right)=\overline{\operatorname{co}} g_{2}\left(\operatorname{cl} G_{2} \cap K_{1}\right)$. But $\operatorname{cl} G_{2} \cap K_{1}$ is compact and $g_{2}$ is continuous, so $g_{2}\left(\mathrm{cl} G_{2} \bigcap K_{1}\right)$ is compact and $K_{2}$ is compact. Since $K_{1} \supset K_{\text {odd }}$ and $K_{2} \supset K_{\text {even }}, K_{\text {odd }}$ and $K_{\text {even }}$ are compact. 
In the general case, recall that $K_{1}=\overline{\operatorname{co}} g_{1}\left(G_{1}\right), K_{2}=\overline{\operatorname{co}} g_{2}\left(G_{1} \cap K_{1}\right)$ and generally, $K_{2 n-1}=\overline{\operatorname{co}}\left(G_{1} \cap K_{2 n-2}\right)$ and $K_{2 n}=\overline{\operatorname{cog}} g_{2}\left(G_{2} \cap K_{2 n-1}\right)$. Thus we have $\gamma\left(K_{1}\right) \leq k_{1} \gamma\left(G_{1}\right), \gamma\left(K_{2}\right) \leq k_{2} k_{1} \gamma\left(G_{1}\right)$ and, generally, $\gamma\left(K_{2 n-1}\right) \leq k_{1}\left(k_{1} k_{2}\right)^{n-1} \gamma\left(G_{1}\right)$ and $\gamma\left(K_{2 n}\right) \leq\left(k_{1} k_{2}\right)^{n} \gamma\left(G_{1}\right)$. Since $k_{1} k_{2}<1, \gamma\left(K_{2 n-1}\right)$ and $\gamma\left(K_{2 n}\right)$ opproach zero, so $K_{\text {odd }}$ and $K_{\text {even }}$ are compact.

Q.E.D.

The reader may have noticed that while we have generalized the additivity, commutativity, and homotopy properties for the ordinary fixed point index to the context of $k$-set-contractions, we have avoided any attempts at generalizing the normalization property. We shall now remedy this omission. Our goal is to establish the following theorem,

Theorem 4. - (Normalization property). Let $X \in$ of be a metric ANR and suppose that $X \subset B, B$ a $\mathrm{B}_{\triangle \mathrm{ANACH}}$ space, and $X$ inherits its metric from the norm on $B$. Suppose that $f: X \rightarrow X$ is a $k$-set-contraction, $k<1$, and assume that $f^{n}(X)$ is bounded for some $n \geq 1$. Then $\Lambda_{\text {gen }}(f)$, LerAY's generalized Lefschetz number, is defined (using either singular or $\overline{\mathrm{C}}_{\mathrm{EOH}}$ homology) and $\Lambda_{\text {gen }}(f)=i_{X}(f, X)$.

It is likely that Theorem 4 can be generalized, but the theorem as stated will suffice for our purposes.

In order to prove Theorem 4, we shall need a number of lemmas. Let us begin with some simple linear algebra and show that two definitions of a generalized trace, one due to Browder [8] and one to Leray [23], are equivalent.

(A) Brownen's definition: Let $V$ be a vector space and $S: \nabla \rightarrow V$ a linear map. Suppose there exists a finite dimensional subspace $E$ such that $S: E \rightarrow E$ and such that for every $v \in V, S^{m}(v) \in E$ for some $m$ depending on $v$. Define $\operatorname{tr}_{\text {gen }}(S)=\operatorname{tr}(S \mid E)$.

(B) LerAY's definition: Let $V$ be a vector space and $S: V \rightarrow V$ a linear map. Let $N=\bigcup_{j=1}^{\infty} S^{-j}(0)$ and suppose $V / N$ is finite dimensional. Since $S: N \rightarrow N$ we have $\bar{S}: V / N \rightarrow V / N$. Define $\operatorname{tr}_{\mathrm{gen}}(S)=\operatorname{tr}(\bar{S})$.

Lema 2. - Definitions $A$ and $B$ are equivalent.

Proof. - $A \Rightarrow B$. Suppose we have a finite dimensional subspace $E$ as in definition $A$. Since $E$ is finite dimensional, we can find $n$ such that $S: S^{n}$ $(E) \simeq S^{n+1}(E)$ is an isomosphism. Let us write $F=S^{n}(E)$. Giren $v \in \nabla, S^{m}(v) \in E$ from some $m$, so $S^{n+m}(v)=x \in F$. Since $S \mid F$ is an isomorphism, we ean find $z \in F$ such that $S^{n+m}(z)=x=S^{n+m}(v)$. Let us define $\theta(v)=z$. We claim that $\theta(v)$ is well defined. For suppose $S^{n_{1}}(v) \in F$ and $S^{n_{2}}(v) \in F$, and we select $z_{1}$ and $z_{2}$ in $F$.such that $S^{n_{1}}\left(z_{1}\right)=S^{n_{1}}(v)$ and $S^{n_{2}}\left(z_{2}\right)=S^{n_{2}}(v)$. We can assume that $n_{2} \geq n_{1}$, so we have $S^{n_{2}-n_{1}}\left(S^{n_{2}}\left(z_{1}\right)\right)=S^{n_{2}}\left(z_{1}\right)=S^{n_{2}}\left(z_{2}\right)$. Since $S$ is an isomorphism on $F, z_{1}=z_{2}$, and $\theta$ is well defined. Clearly, $\theta$ is linear. 
Consider $V / N$, where $N$ is as in definition $B$. It is easy to see that $\theta(N)=0$, so we obtain $\bar{\theta}: \nabla / N \rightarrow F$. To see that $\bar{\theta}$ is an isomorphism, it suf. fices to show that $\theta^{-1}(0)=N$. Take $x \in V-N$ and select $m$ so that $S^{m}(x) \in F$. Since $x \notin N, S^{m}(x) \neq 0$; and since $S \mid F$ is an isomorphism, if we take $z \in F$ such that $S^{m}(z)=S^{m}(x) \neq 0, z \neq 0$, i.e., $\theta(x) \neq 0$. Thus we see that $\dot{\theta}: V / N \rightarrow S^{n}(E)$ is an isomorphism.

Finally, notice that we have commutativity in the following diagram:

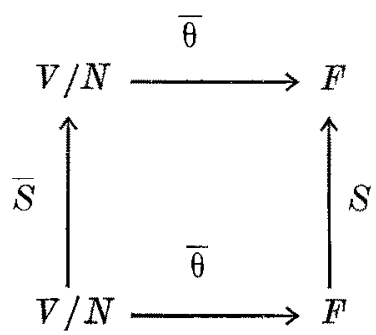
trace.

It follows that $\operatorname{tr}(\bar{S})=\operatorname{tr}\left(\bar{\theta}^{-1}(S \mid F(\bar{\theta})=\operatorname{tr}(S \mid F)=\right.$ BRow DER's generalized

Conversely, let us show that $B \Rightarrow A$. Suppose that $\nabla / N$ is finite dimensional and let $\left[v_{1}\right], \ldots,\left[v_{n}\right]$ be a basis; $[v]$ denotes the equivalence class of $v \in V$ in $V / N$. By definition, $S v_{i}-\sum_{j=1}^{n} a_{i j} v_{j} \in N$ for some $a_{i j}, 1 \leq j \leq n$, so $S^{m_{i}}\left(S v_{i}-\sum_{j=1}^{n} a_{i j} v_{j}\right)=0$ and $S^{m_{i}+1} v_{i}=\sum_{j=1}^{n} a_{i j} S^{m}{ }_{i} v_{j}$. Select $m \geq \max \left\{m_{1}, \ldots, m_{n}\right\}$ and consider the subspace $E$ spanned by $S^{j} v_{i}, 1 \leq i \leq n, 0 \leq j \leq m$. Since $S^{m+1} v_{i}=\sum_{j=1}^{n} a_{i j} S^{m} v_{j}, S$ maps $E$ into itself. Given $v \in V$, by definition $v-\sum_{j=1}^{n}$ $b_{i} v_{i} \in N$ for some $b_{i}, 1 \leq i \leq n$; and we find $S^{k} v=\sum_{i=1}^{n} b_{i} S^{k} v_{i} \in E$ for some $k \geq 0$. It follows that $E$ meets the conditions of definition $A$. Now just use the proof that $A \Rightarrow B$ to show $\operatorname{tr}(\bar{S})=\operatorname{tr}(S \mid E)$, where $\bar{S}: V / N \rightarrow V / N$.

Q.E.D.

If $X$ is a topological space, $f: X \rightarrow X$ a continuous map, $H_{*, i}(X)$ (coefficients in the rationals) is a vector space and $f_{*, i}$ a linear map. We define $\Lambda_{\text {gen }}(f)=\sum_{i \geq 0}(-1)^{i} t r_{\text {gen }}\left(f f_{, i}\right)$, where we assume $\operatorname{tr}_{\text {gen }}\left(f_{q, i}\right)$ is defined for all $i$ and zero except for finitely many $i$. The above lemma shows we can use either LERAY's or BRowder's definition of the generalized trace. We shall use Browder's definition. Our next lemma is due to Leray, we prove if for completeness.

Lemma 3. - Suppose we have $\nabla \stackrel{S}{\rightarrow} W \stackrel{T}{\rightarrow} \nabla$, where $\nabla$ and $W$ are vector spaces. $S$ and $T$ are linear maps. If $t r_{\text {gen }}(T S)$ is defined, $t r_{\text {gen }}(S T)$ is defined and $t r_{\mathrm{gen}}(T S)=t r_{\mathrm{gen}}(S T)$.

Proof. - Assume $\operatorname{tr}_{\text {gen }}(T S)$ is defined and select a finite dimensional subspace $E$ of $V$ which meets the conditions of definition $A$ for the linear map $T S$. Let $F=S(E) \subset W$. Clearly, $F$ is finite dimensional, and $S T: F \rightarrow F$ 
since $S T(F)=S T(S(E))=S(T S(E)) \subset S(E)=F$. If $w \in W$, by definition of $E$ there exists an $m$ such that $(T S)^{m}(T w) \in E$, so $(S T)^{m+1}(w)=S(T S)^{m}(T w) \in S(E)=F$. Thus ${ }_{S} \operatorname{tr}_{\mathrm{gen}}(S T)$ is defined and $\operatorname{lr}_{\mathrm{gen}}(S T)=\operatorname{tr}(S T \mid F)$. However, we have $E \stackrel{S}{\rightarrow} F \stackrel{T}{\rightarrow} E$, so the usual commutativity property implies $t r_{\mathrm{gen}}(T S)=\operatorname{tr}(T S \mid E)=$ $=\operatorname{tr}(S T \mid F)=\operatorname{tr}_{\mathrm{gen}}(S T)$.

Lemma 4. - Let $X$ be a topologicel space and $f: X \rightarrow X$ be a continuous map. Let $Y$ be an subspace of $X$ such that $f$ maps $Y$ into itself and $Y \supset f(X)$. Then $\Lambda_{\text {gen }}(f)$ is defined if and only $\Lambda_{\text {gen }}(f \mid Y)$ is defined, and $\Lambda_{\text {gen }}$ $(f)=\Lambda_{\text {gen }}(f \mid Y) . f \mid Y$ is meant to indicate a map from $Y$ to $Y$.

Proof. - Consider the diagram

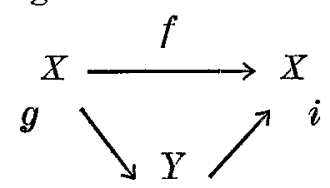

where $i$ denotes inclusion and $g$ denotes $f$ viewed as a map from $X$ to $Y$. We have $t_{*, j}=i_{*, j} g_{*, j}: H_{j}(X) \rightarrow H_{j}(X)$. However $g_{*, j}: H_{j}(X) \rightarrow H_{j}(Y)$ and $i_{*, j}: H_{j}(Y) \rightarrow H_{j}(X)$, so Lemma 3 implies that $\operatorname{tr}_{\text {gen }}\left(t_{*, j}\right)=\operatorname{tr}_{\text {gen }}\left(g_{*_{, j}} i_{*_{j}}\right)$. Since $g_{*, j} i_{*, j}=(f \mid Y)_{*, j}, \operatorname{tr}_{\mathrm{gen}}\left(f_{*, j}\right)=\operatorname{tr}_{\mathrm{gen}}((f \mid Y) *, j)$, and it follows that $\Lambda_{\mathrm{gen}}(f)=\Lambda_{\mathrm{gen}}(f \mid Y)$.

Q.E.D.

Lemma 5. - Let $X$ be a topological space and $f: X \rightarrow X$ a continuous map. Let $G$ be a subspace of $X$ and suppose $f: G \rightarrow G$ and $f^{n}(X) \subset G$ for some $n \geq 1$. Then $\Lambda_{\text {gen }}(f)$ is defined if and only if $\Lambda_{\text {gen }}(f \mid G)$ is defined and $\Lambda_{\text {gen }}(f)=\Lambda_{\text {gen }}(f \mid G)$.

Proor. - Let $G_{i}=f^{i}(X) \cup G, 1 \leq i \leq n$, and since $f\left(G_{i}\right) \subset G_{i}$, write $f \mid G_{i}$, which we will view as a map from $G_{i}$ to $G_{i}$. Applying Lemma 3 repeatedly, we find that $\Lambda_{\text {gen }}(f)=\Lambda_{\text {gen }}\left(f \mid G_{1}\right)=\ldots=\Lambda_{\text {gen }}\left(f \mid G_{n}\right)=\Lambda_{\text {gen }}(f \mid G) . \quad$ Q.E.D.

Up until now our lemmas have been very general. Our next lemma makes use of the hypotheses of Theorem 4. We shall not prove Lemma 6 here, since it appears more naturally in a sequence of results in [32].

Lemma 6, - Under the hypotheses of Theorem 4, there exists a bounded open neighborhood $G$ of $f^{n}(X)$ such that $\operatorname{cl}(f(G)) \subset G$.

Now let $G$ be as in Lemma 6 and let $K_{m}^{*}=\overline{\mathrm{co}} f\left(G \cap K_{m-1}^{*}\right) \cap X$ and $K_{1}^{*}=\overline{\mathrm{co}} f(G) \cap X$. If we write $G_{m}=G \cap K_{m}^{*}$ and $G_{0}=G$, we see that $f: G_{m} \rightarrow G_{m+1}$ and $G_{m} \supset G_{m+1}$, so that by Lemma 3 we have $\Lambda_{\text {gen }}(f \mid G)$ is defined iff $\Lambda_{\text {gen }}\left(f \mid G_{m}\right)$ is defined $\left(f \mid G_{m}\right.$ is viewed as a map from $G_{m}$ to $\left.G_{m}\right)$ and $\Lambda_{\text {gen }}(f \mid G)=\Lambda_{\text {gen }}\left(f \mid G_{m}\right)$. Thus in order to prove Theorem 4 , it suffices to show that $\Lambda_{\text {gen }}\left(f \mid G \cap K_{m}^{*}\right)$ is defined and equal to $i_{X}(t, X)$ for $m$ large enough.

Lemma 7. - We can find $\delta>0$ and $m_{1}$ such that for $m \geq m_{1}, N_{\delta}(f(\operatorname{cl} G \cap$ $\left.\left.\left.\cap K_{m}^{*}\right)\right)\right) \subset G$. 
Proof. - Let us set $K_{\infty}^{*}=\cap_{m \geq 1} K_{m}^{*}$. We then have $\operatorname{cl}(G) \cap K_{\infty}^{*}=\cap_{m \geq 1}$ $\operatorname{cl}(G) \cap K_{m}^{*}$, and since $\gamma\left(\operatorname{cl}(G) \cap K_{m}^{*}\right) \rightarrow 0$, Proposition 2 of $I-A$ shows that given any open neighborhood $V$ of $\operatorname{cl}(G) \cap K_{\infty}^{*}, \operatorname{cl}(G) \cap K_{m}^{*} \subset V$ for $m \geq m_{1}$. Since $f\left(\operatorname{cl}(G) \cap K_{\infty}^{*}\right)$ is a compact subset of $G$, we can find $\delta>0$ such that $N_{2 \delta}\left(f\left(\operatorname{cl}(G) \cap K_{\infty}\right)\right) \subset G$. Setting $V=f^{-1}\left(N_{\delta}\left(f\left(\operatorname{cl}(G) \cap K_{\infty}^{*}\right)\right)\right)$, which is an open neighborhood of $\operatorname{cl}(G) \cap K_{\infty}^{*}$, for $m \geq m_{1}$ we have $\mathrm{cl}(G) \cap K_{m}^{*} \subset \nabla$, so $N_{\delta}(f$ icl $\left.\left.\left(G \cap K_{m}^{*}\right)\right)\right) \subset N_{\delta}\left(f\left(\mathrm{cl}(G) \cap K_{m}^{*}\right)\right) \subset N_{2 \delta}\left(f\left(\operatorname{cl}(G) \cap K_{\infty}^{*}\right)\right) \subset G$.

Q.E.D.

We can now prove Theorem 4. Select $\delta$ and $m_{1}$ as in Lemma 7 . Suppose that $X=\cup_{i \in J} C_{i}$ is a locally finite union of closed, comvex sets in $B$. Since $K_{\infty}^{*}$ is a compact subset of $X$, let $W$ be an open neighborhood of $K_{\infty}^{*}$ such that $W \cap C_{i}=\emptyset$ unless $i \in F$, where $F$ is some finite subset of $J$. Select $m_{2}$ such that for $m \geq \mathbf{m}_{2}, K_{m}^{*} \subset W$, as we can do, since $\gamma\left(K_{m}^{*}\right) \rightarrow 0$. It follows that for $m \geq m_{2}, K_{m}^{*}=\cup_{i \in F}\left(\overline{\operatorname{co}} f\left(G \cap K_{m-1}^{*}\right)\right) \cap C_{i}$, a finite union of closed, convex sets. It is clear that Corollary 2 , Section $B$, applies here, so for $\delta$ as above and $m \geq m_{3}$, we can find a deformation retraction $H_{m}: K_{m}^{*} \times I \rightarrow K_{m}^{*}$, $H_{m}(x, t)=x$ for $\left.x \in K, t \in \underset{\infty}{*} I, H_{m^{\prime}} \cdot, 1\right)$ a retraction onto $K_{\infty}^{*}$, and $\left\|H_{m}(x, t)-x\right\|<\delta$ for $x \in K_{m}^{*}, t \in I$. For $m \geq \max \left\{m_{1}, m_{3}\right\}$ and $x \in \operatorname{cl}\left(G \cap K_{m}^{*}\right)$, consider the homotopy $F_{m}(x, t)=H_{m}(f(x), t), 0 \leq t \leq 1$. It is clear that $F_{m}(x, t) \in K_{m}^{*}$, and since $\left\|H_{m}(f(x), t)-f(x)\right\|<\delta$ and $N_{\delta}\left(f\left(\mathrm{cl}\left(G \cap K_{m}^{*}\right)\right) \subset G, H_{m}(f(x), t) \in G\right.$. It follows that $F_{m}:\left(G \cap K_{m}^{*}\right) \times I \rightarrow G \cap K_{m}^{*}$, so the maps $f_{t}: G \cap K_{m}^{*} \rightarrow G \cap K_{m}^{*}$ defined by $f_{t}(x)=F_{m}(x, t)$ induce the same map in homology; and if we write $r_{m}=H_{m}(\cdot, 1)$, which is a retraction of $K_{m}^{*}$ onto $K_{\infty}^{*},\left(r_{m} f\right): G \cap K_{m}^{*} \rightarrow G \cap K_{m}^{*}$ and $f: G \cap K_{m}^{*} \rightarrow$ $\rightarrow G \cap K_{m}^{*}$ induce the same maps in homology. It follows that $\Lambda_{\operatorname{gen}}\left(f \mid G \cap K_{m}^{*}\right)=$ $=\Lambda_{\text {gen }}\left(r_{m} t \mid G \cap K_{m}^{*}\right)$, so it suffices to show that $\Lambda_{\text {gen }}\left(r_{m} t \mid G \cap K_{m}^{*}\right)$ is defined and equal to $i_{X}(f, X)$. However, $\left(r_{m} f\right)\left(G \cap K_{m}^{*}\right) \subset G \cap K_{\infty}^{*}$, so by Lemma 4 we obtain $\Lambda_{\text {gen }}\left(r_{m} f \mid G \cap K_{m}^{*}\right)=\Lambda_{\text {gen }}\left(r_{m} f \mid G \cap K_{\infty}^{*}\right)=\Lambda_{\text {gen }}\left(f \mid G \cap K_{\infty}^{*}\right)$.

We are almost done. By the additivity property, $i_{X}(f, X)=i_{X}(f, G) \equiv$ $\equiv i_{K_{\infty}^{*}}\left(t, G \cap K_{\infty}\right)$. Since $\operatorname{cl}\left(f\left(G \cap K_{\infty}^{*}\right)\right)$ is a compact subset of $G \cap K_{\infty}^{*}$, we can find an open neighborhood $U$ in $G \cap \mathrm{K}_{\infty}^{*}$ of $\operatorname{cl}\left(f\left(G \cap K_{\infty}^{*}\right)\right)$ such that $\operatorname{cl}(U)$ is a compact subset of $G \cap K_{\infty}^{*}$. Cover $\operatorname{cl}(U)$ by a finite number of compact, convex sets $D_{i} \subset G \cap K_{\infty}^{*}, 1 \leq i \leq n$, and let $A=\bigcup_{i=1}^{n} D_{i}$. Then $A$ is a compact $A N R, f(A) \subset A$, and in fact $f\left(G \cap K_{\infty}^{*}\right) \subset A$. By Lemma 3 , we have $\Lambda_{\text {gen }}\left(f \mid G \cap K_{\infty}^{*}\right)=\Lambda_{\text {gen }}(f \mid A)$, which we know is defined. On the other hand, since all fixed points of $t$ in $G \cap K^{*}$ lie in $U, i_{K_{\infty}^{*}}\left(t, G \cap K^{*}\right)=i_{K_{\infty}^{*}}(f, U)$. Furthermore, by the normalization property, $\Lambda_{\text {gen }}(f \mid A)=\Lambda(f \mid A)=i_{A}(t, A)=$ $=i_{A}(f, U)=i_{K_{\infty}^{*}}(t, U)$, since $f(U) \subset A$ and $U \cap A=U$. This shows that $\Lambda_{\mathrm{gen}}(f \mid A)=i_{X}(f, X)$. 


\section{F. - The fixed point index for 1-set-contractions.}

In this section we shall define a fixed point index for maps which are essentially 1-set-contractions and which satisfy certain additional conditions. Our main interest will be in a corollary of this work, the fixed point index for condensing maps, but we would save no effort by initially restricting ourselves to that case. Specifically, we consider the following situation: Suppose $X \in \mathscr{F}, G$ is an open subset of $X$, and $g: G \rightarrow X$ is a continuous map. We shall say that $g$ is an admissible map for the fixed point index or simply an admissible map iff

(1) $S=\{x \in G: g(x)=x\}$ is closed and bounded.

(2) There exists a bounded, open neighborhood $U$ of $S$ with $\operatorname{cl}(U) \subset G$ and a locally finite covering $\left\{C_{j}: j \in J\right\}$ of $X$ by closed, convex sets $C_{j} \subset X$ such that $(a) g \mid \mathrm{cl}(U)$ is a 1 -set-contraction, $(b) I-g \mid \mathrm{cl}(U)$ is a closed map $(I=$ the identity on the BANAOH space $B$ containing $X)$, and $(c) g(\mathrm{cl}(U)) \cap C_{j}$ is empty except for finitely many $j \in J$. If $S$ is empty, $U$ may be empty. If $g, U$, and $\left\{C_{j}: j \in J\right\}$ are as above, we shall say that $\left\langle g, U,\left\{O_{j}: j \in J\right\}\right\rangle$ is an admissible triple.

Before proceding further let us consider some classes of admissible maps. If $G$ is a bounded, open subset of a $B_{A N A C H}$ space $X$ and $g: \operatorname{cl}(G) \rightarrow X$ is a 1-set-contraction such that $g(x) \neq x$ for $x \in \partial G$ and $I-g \mid \operatorname{cl}(G)$ is a closed map, then $g$ is an admissible map. To give another example we need the following lemma.

Lemma 1. - Let $A$ be a olosed, bounded subset of a BaNaCH space $B$. Let $g: A \rightarrow B$ be a condensing map. Then $(I-g)$ is a proper map.

Proof. - Let $M$ be a compact subset of $B$. We must show that $N=\{x \in A$ : $(I-g)(x) \in M\}$ is compact. Clearly $N$ is closed, so it suffices to show $\gamma(N)=0$. If $x \in N, x=g(x)+m$ for some $m \in M$, and thus $N \subset g(N)+M$. If $\gamma(N)>0$, $\gamma(N) \leq \gamma(g(N))+\gamma(M)=\gamma(g(N))<\gamma(N)$, a contradiction.

Q.E.D.

Proposition 1. - Let $X \in F$ F and let $G$ be an open subset of $F$. Let $g: G \rightarrow X$ be a local condensing map such that $S=\{x \in G: g(x)=x\}$ is com. pact. Then $g$ is an admissible map. In particular, if $G$ is bounded, and $g: \mathrm{ol}(G) \rightarrow X$ is a condensing map such that $g(x) \neq x$ for $x \in \partial G$, then $S$ is compact, so that $g$ is admissible in that case.

Proof. - Since $S$ is compact and $g$ is a local condensing map, there exists an open neighborhood $V$ of $S$ such that $\operatorname{cl}(V) \subset G$ and $g \mid \operatorname{cl}(V)$ is a condensing map. Clearly $g \mid \operatorname{cl}(V)$ is a 1 -set-contraction, and by Lemma 1 , $I-g \mid \operatorname{cl}(\nabla)$ is a proper map and hence closed. Since $X \in \mathscr{F}$, there exists a 
locally finite covering of $X,\left\{C_{j}: j \in J\right\}$, by closed, convex sets $C_{j} \subset X$. Since $S=g(S)$ is compact and the covering is locally finite, there exists an open neighborhood 0 of $g(S)$ such that $\mathrm{el}(0) \cap C_{j}$ is empty for finitely many $j$. Setting $W=g^{-1}(0), g(\mathrm{cl}(W)) \cap C_{j}$ is empty except for finitely many $j$. If we let $U=V \cap W$, it is easy to see that $\left\langle g, U,\left\{C_{j}: j \in J\right\}>\right.$ is an admissible triple and $g$ is an admissible map.

The last statement of the theorem is clear since by Lemma $1,\{x \in \operatorname{cl}(G)$ : $g(x)=x\}$ is compact and $\{x \in \mathrm{cl}(G): g(x)=x\}=S . \quad$ Q.E.D.

Now let $g$ be an admissible map as in the first paragraph and let $<g, U,\left\{C_{j}: j \in J\right\}>$ be an admissible triple. Since $(I-g)(x) \neq 0$ for $x \in \partial U$ and since $(I-g) \mid \operatorname{cl}(U)$ is closed map, inf $\{\|(I-g)(x)\|: x \in \partial U\}=\delta>0$. If $f: \operatorname{cl}(U) \rightarrow X$ is a continuous map, we shall say that $f$ is an admissible approximation with respect to $<g, U,\left\{C_{j}: j \in J\right\}>$ if (1) $t$ is a $k$-set-contraction, $k<1$.

(2) $\|f(x)-g(x)\|<\delta$ for $x \in \operatorname{cl}(U), \delta=\inf \{\|(I-g)(x)\|: x \in \partial U\}$.

(3) For all $j \in J$ and $x \in \operatorname{cl}(U)$, if $g(x) \in C_{j}$, then $f(x) \in C_{j}$.

Theorem 1. - Let $G$ be an open subset of $X \in \mathscr{F}, g: G \rightarrow X$ an admissible map. Let $\left\langle g, U,\left\{C_{j}: j \in J\right\}\right\rangle$ be an admissible triple. Then given $\eta>0$, there exists an admissible approximation $f$ with respect to $\left\langle g, U,\left\{C_{j}: j \in J\right\}>\right.$ such that $\|g(x)-f(x)\|<\eta$ for $x \in \operatorname{cl}(U)$. Furthermore if $\left\langle g, V,\left\{D_{l}: l \in L\right\}\right\rangle$ is another admissible triple and $h$ is an admissible approximation with respect to $\left\langle g, \nabla,\left\{D_{l}: l \in L\right\}\right\rangle$, then $i_{X}(h, \nabla)=i_{X}(f, U)$. (Notice that the assumption that $h$ and $f$ are admissible approximations guarantes that $i_{X}(h, \nabla)$ and $i_{X}(f, U)$ are defined).

Proof. - First we show that admissible approximations exist. By assumption $g(\operatorname{cl}(U)) \cap C_{j}$ is empty unless $j \in F, F$ some finite subset of $J$. Consider $C=\cup_{j \in F} C_{j}$. By Corollary 1, $I-B$, there exists a retraction $R: C \rightarrow K$, where $K$ is some compact subset of $C$, such that $R(y) \in C_{j}$ if $y \in C_{j}$ for all $y \in C, j \in F$. Since $g \mid \operatorname{cl}(U)$ is a 1-set-contraction and $\mathrm{cl}(U)$ is bounded, $\|g(x)\| \leq M$ for $x \in \mathrm{cl}(U)$; and since $K$ is compact, we can also assume $\|y\| \leq M$ for $y \in K$. Let $\delta=\inf \{\|x-g(x)\|: x \in \partial U\}$ and for $0<\eta<\delta$ and $1-\eta / 2 M<$ $<t<1$, define $f(x)=\operatorname{tg}(x)+(1-t) R(g(x))$. From our construction it is clear that $\|f(x)-g(x)\|<\eta$ for $x \in \operatorname{cl}(U)$. Since $g$ is a 1 -set-contraction and $R$ is compact, $f$ is a $t$-set-contraction, $t<1$. Finally, since $R(y) \in C_{j}$ if $y \in C_{j}$ for $j \in F$ and since $C_{j}^{r}$ is convex, $f(x) \in C_{j}$ if $g(x) \in C_{j}$.

We next have to show that $i_{X}(h, \nabla)=i_{X}(f, V)$. Since $I-g \mid \mathrm{cl}(V)$ is a clo. sed map and $(I-g)(x) \neq 0$ for $\in \operatorname{cl}(\nabla)-U \cap V=V_{1}$, inf $\left\{\|(I-g)(x)\|: x \in V_{1}\right\}=$ $=\eta>0$. (Notice that $U \cap V$ may be empty if $S$ is empty). Using the first part of this theorem, let $h^{\prime}$ be an admissible approximation with respect to 
$<g, V,\left\{D_{l}: l \in L\right\}>$ such that $\left\|h^{\prime}(x)-g(x)\right\|<\eta$ for $x \in \operatorname{cl}(\nabla)$. Consider the homotopy $h_{s}=s h+(1-s) h^{\prime}, 0 \leq s \leq 1$. Since $\|h(x)-g(x)\|$ and $\left\|h^{\prime}(x)-g(x)\right\|$ are strictly less than $\|x-g(x)\|$ for $x \in \partial V, h_{s}(x) \neq x$ for $x \in \partial V$. Since $h(x)$ and $h^{\prime}(x)$ both lie in $C_{j} \ni g(x), h_{s}(x) \in X$. Thus the homotopy is permissible and $i_{X}(h, V)=i_{X}\left(h^{\prime}, \nabla\right)$. However, $h^{\prime}(x) \neq x$ for $x \in \nabla_{1}$, so $i_{X}\left(h^{\prime}, U \cap V\right)=i_{X}\left(h^{\prime}, \nabla\right)$. Similarly, $\left.i_{X}(t, U)=i_{X} f^{\prime}, U \cap \nabla\right)$, where $f^{\prime}$ is an admissible approximation with respect to $\left\langle g, U \cap \nabla,\left\{O_{j}: j \in J\right\}\right\rangle$. Thus from the start we may as well assume that $f$ and $h$ are admissible approximations with respect to $\left\langle g, U \cap V,\left\{C_{j}: j \in J\right\}\right\rangle$ and $\left\langle g, U \cap \nabla,\left\{D_{l}: l \in L\right\}\right\rangle$ respectively and try to prove $i_{X}(f, U \cap V)=i_{X}(h, U \cap \nabla)$.

Consider the admissible triple $<g, U \cap V,\left|C_{j} \cap D_{l}:(j, l) \in J \times L\right|>$ and and let $\theta$ be an admissible approximation with respect to this triple. We elaim that $i_{X}(t, U \cap V)=i_{X}(\theta, U \cap V)=i_{X}(h, U \cap V)$. It suffices to prove the first equality, the proof of the other being the same. Consider the homotopy $\theta_{s}(x)=(1-s) \theta(x)+s f(x), 0 \leq s \leq 1$. Just as above, $\theta_{s}(x) \neq x$ for $x \in \mathfrak{\partial}(U \cap V)$, $0 \leq s \leq 1$. Further, $\theta_{s}(x) \in X$ for $x \in \operatorname{cl}(U \cap \nabla)$ and $0 \leq s \leq 1$. For if $g(x) \in C_{j} \cap D_{l}$, $\theta(x) \in C_{j} \cap D_{l} \subset C_{j}$ and $f(x) \in C_{j}$, so $\theta_{s}(x) \in C_{j}$. Thus the homotopy is permissible and $i_{X}(f, U \cap V)=i_{X}(\theta, U \cap V)$.

Q.E.D.

Definition. - Let $G$ be an open subset of a space $X \in \mathscr{F}$ and let $g: G \rightarrow X$ be a continuous function which is admissible. Let $\left\langle g, D,\left\{C_{j}: j \in J\right\}\right\rangle$ be an admissible triple and let $f$ be an admissible approximation with respect to this triple. We define $i_{X}(g, G)=i_{X}(f, U)$. Theorem 1 shows this definition is well-defined.

Theorem 1 (Additivity property). - Let $G$ be an open subset of a space $X \in \mathbb{F}$ and $g: G \rightarrow X$ be an admissible map. Let $S=\{x \in G \mid g(x)=x\}$ and as. sume $S \subset G_{1} \cup G_{2}, G_{1}$ and $G_{2}$ disjoint open subsets of $G$. Then $g$ is admissible as a map from $G_{i}$ to $X(i=1,2)$ and $i_{X}(g, G)=i_{X}\left(g, G_{1}\right)+i_{X}\left(g, G_{2}\right)$. Further, if $i_{X}(g, G) \neq 0$, then $g$ has a fixed point in $G$.

Proor. - Since $g$ is an admissible map, let $\left\langle g, U,\left\{C_{j}: j \in J\right\}\right\rangle$ be an admissible triple. Let $S=\{x \in G: g(x)=x\}$ and let $S_{i}=S \cap G_{i}, 1 \leq i \leq 2$. $S_{i}$ is bounded since $S$ is bonnded and $S_{i}$ is closed since $S_{1}=S \cap G_{2}^{\prime}$ and $S_{2}=S \cap G_{1}^{\prime}, G_{i}^{\prime}=$ complement of $G$. Let $U_{i}$ be an open neighborhood of $S_{i}$ such that $\operatorname{el}\left(U_{i}\right) \subset G_{i} \cap U$. It is clear that $\left\langle g \mid G_{i}, U_{i},\left\{C_{j}: j \in J\right\}\right\rangle, i=1,2$, are admissible triples. Let $\delta=\inf \left\{\|x-g(x)\|: x \in \operatorname{cl}(U) \sim\left(U_{1} \cup U_{2}\right)\right\}$ and let $h$ be an admissible approximation with respect to $\left\langle g, U,\left|C_{j}: j \in J\right|\right\rangle$ such that $\|h(x)-g(x)\|<\delta$ for $x \in \mathrm{cl}(U)$. Then we have $i_{X}(g, G)=i_{X}(h, U)=i_{X}\left(h, U_{1}\right)+$ $+i_{X}\left(h, U_{2}\right)=i_{X}\left(g, G_{1}\right)+i_{X}\left(g, G_{2}\right)$.

If $i_{X}(g, G) \neq 0$, there must exist an admissible triple $\left\langle g, U,\left\{O_{j}: j \in J\right\}\right\rangle$ such that $U$ is nonempty and an admissible approximation $h$ with respect to this admissible triple, such that $i_{X}(h, U) \neq 0$. By Theorem 1 , there exists a 
sequence of admissible approximations $h_{n}$ with respect to $\left\langle g, U,\left\{C_{j}: j \in J\right\}\right\rangle$ such that $\sup \left\{\left\|g(x)-h_{n}(x)\right\|: x \in \mathrm{cl}(U)\right\} \rightarrow 0$ and $i_{X}\left(h_{n}, U\right)=i_{X}(h, U) \neq 0$. Thus $h_{n}$ has a fixed point $x_{n} \in \mathrm{cl}(U)$ and $x_{n}-g\left(x_{n}\right) \rightarrow 0$. Since $I-g \mid \operatorname{cl}(U)$ is a closed map, $g$ has a fixed point in $\operatorname{cl}(U)$.

Q.E.D.

Before proceeding further we need to define the concept of an admissi. ble homotopy for the fixed point index. Let $J=[0,1]$, the unit interval, and let $\Omega$ be an open subset of $X \times J, X \in F$. We shall say that a continuous map $F: \Omega \rightarrow X$ is an admissible homolopy if (1) $S \equiv\{(x, t) \in \Omega: F(x, t)=x\}$ is closed and bounded. (2) There exists a bounded open neighborhood $U$ of $S$ with $\operatorname{cl}(U) \subset \Omega$ and a locally finite covering $\left\{C_{l}: k \in K\right\}$ of $X$ by closed, convex sets $C_{k} \subset X$ such that the following properties hold: (a) For any bounded subset $A$ of $X, \gamma(F((A \times J) \cap \mathrm{cl}(U))) \leq \gamma(A)$. (b) $\Pi-F \mid \mathrm{cl}(U)$ is a closed map, where $\Pi$ is the projection $\Pi(x, t)=x$. (c) $F(\operatorname{cl}(U)) \cap C_{k}$ is empty except for finitely many $k$. If $F, U$, and $\left\{C_{k}: k \in K\right\}$ are as above we say $\left\langle F, U,\left\{C_{k}: k \in K\right\}>\right.$ is a homotopy admissible triple.

Theorem 3. (Homotopy property). - Let $\Omega$ be an open subset of $X \times J$, $X \in \mathscr{F}, J=[0,1]$, and let $F: \Omega \rightarrow X$ be admissible. Then if we write $\Omega_{t}=\{x:(x, t) \in \Omega\}$ and $F_{t}=F(\cdot, t), F_{t}: \Omega_{t} \rightarrow X$ is an admissible map and $i_{X}\left(F_{0}, \Omega_{0}\right)=i_{X}\left(F_{1}, \Omega_{1}\right)$.

Proof. - If $\left\langle F, U,\left\{C_{k}: k \in K\right\}\right\rangle$ is a homotopy admissible triple, it is clear that $\left\langle F_{t}, U_{t},\left\{C_{k}: k \in K\right\}>\right.$ is an admissible triple $\left(U_{t}=\{x:(x, t) \in U\}\right)$, so $F_{t}: \Omega_{t} \rightarrow Y$ is an admissible map. Let $\delta=\inf \|\|(I I-F)(x, t) \|:(x, t) \in \operatorname{cl}(U) \sim U t$ and notice that by our hypotheses, $\delta>0$. By assumption $F(\mathrm{cl} U) \cap C_{k}$ is empty for $k \notin L, L$ some finite subset of $K$. Let $C=\cup_{k \in L} C_{k}$ and let $R: C \rightarrow D$ be a retraction of $C$ onto a compact subset $D$ of $C$ such that $R(x) \in C_{k}$ if $x \in C_{k}$. ( $R$ exists by Corollary 1 , Section $B$ ). It is easy to show that there exists $M$ such that $\|F(x, t)\| \leq M$ for $(x, t) \in \operatorname{cl}(U)$ and $\|y\| \leq M$ for $y \in D$. Select $s$ such that $1-\frac{\delta}{2 M}<s<1$ and define $\left.H(x, t)=s F(x, t)\right)+(1-3) R(x)$. It is easy to see that $\|H(x, t)-F(x, t)\|<\delta$ for $(x, t) \in \operatorname{cl}(U)$, so $H(x, t) \neq x$ for $x \in \operatorname{cl}(U) \sim U$. Our con. struction also implies that if $F(x, t) \in C_{k}$, then $H(x, t) \in C_{k}$. Finally, for any subset $A$ of $X, \gamma(H(A \times J) \cap \operatorname{cl} U)) \leq s \gamma(F((A \times J) \cap \operatorname{cl} U)) \leq s \gamma(A)$, since $R$ is compact. We now apply Corollary 1 , Section $E$, and setting $H_{t}=H(\cdot, t)$, we find $i_{X}\left(H_{0}, U_{0}\right)=i_{X}\left(H_{1}, U_{1}\right)$. However, it is olear that $H_{i}$ is an admissible approximation with respect to $\left\langle H_{t}, U_{t},\left\{C_{k} . k \in K \mid>\right.\right.$, so $i_{X}\left(H_{t}, U_{t}\right)=i_{X}\left(\boldsymbol{F}_{t}, \Omega_{t}\right)$. Thus we have $i_{X}\left(\boldsymbol{F}_{0}, \Omega_{0}\right)=i_{X}\left(F_{1}, \Omega_{1}\right)$.

Q.E.D.

Corollary 1. - Let $G$ be a bounded open subset of $X \in F^{\circ}$ and let $J=[0,1]$. Let $F: \operatorname{cl}(G) \times J \rightarrow K$ be a continuous map such that $F(x, t) \neq x$ for $x \in \partial G, t \in J$. Assume that for any subset $A$ of $\operatorname{cl}(\theta)$ such that $\gamma(A) \neq 0$, $\gamma(F(A \times J))<\gamma(A)$. Then $i_{X}\left(F_{0}, G\right)=i_{X}\left(F_{1}, G\right)$. 
Proof. - It suffices to prove that $F$ is an admissible homotopy. Since $F(x, t) \neq x$ for $(x, t) \in 2 G \times J, S=\{(x, t) \in G: F(x, t)=x\}=\{(x, t) \in \mathrm{c}\}(G): F(x, t)=x\}$ is closed and bounded.

The fact that $\gamma(F((A \cap G) \times J)) \leq \gamma(A)$ for any bounded subset $A$ of $X$ is immediate from our assumptions. We claim that $\Pi-F$ is a closed map, and to prove this it suffices to show $\Pi-F$ is a proper map. Thus let $M$ be any compact subset of $X$ and let $N=(\Pi-F)^{-1}(M)=\{(x, t) \in \operatorname{cl}(G) \times J: x-F$ $(x, t) \in M\}$. Let $N_{1}=\Pi(N)$. Since $N$ is closed and $N \subset N_{1} \times d$, it suffices to show $\gamma\left(N_{1}\right)=0$. However, if $x \in N_{1}, x=F(x, t)+m$ for some $t \in J$ and some $m \in M$. Thus $N_{1} \subset F\left(N_{1} \times J\right)+M$. If $\gamma\left(N_{1}\right)>0$, this would imply $\gamma\left(N_{1}\right) \leq \gamma\left(F^{\prime}\right.$ $\left.\left(N_{1} \times J\right)+M\right)=\gamma\left(F\left(N_{1} \times J\right)\right)<\gamma\left(N_{1}\right)$, a contraction. Thus $\gamma\left(N_{1}\right)=0$ and $\Pi-F$ is proper, $S$ is compact and hence $F(S)$ is compact. Thus if $\left(C_{k}: k \in K\right\}$ is a locally finite covering of $X$ by closed, convex sets, there exists an open nei. ghborhood $W$ of $F(S)$ such that $\operatorname{cl}(W) \cap C_{k}$ is empty, except for finitely many $k$. Setting $U=F^{-1}(W)$ it is clear that $\left\langle F, U,\left\{C_{k}: k \in K\right\}\right\rangle$ is a homotopy admissible triple.

Q.E.D.

Corollary 2. - Let $G$ be a bounded, open subset of $X \in \mathscr{F}$ and let $J=[0,1]$. Let $F: \operatorname{el}(G) \times J \rightarrow X$ be a continuous function such that $F(x, t) \neq x$ for $x \in \partial G \times J$. Assume that $F_{t}=F(\cdot, t)$ is a condensing map for $t \in[0,1]$ and suppose that $F$ is uniformly continuous in $t$. Then for any subset $A$ of $\operatorname{cl}(G)$ with $\gamma(A)>0, \gamma(F(A \times J))<\gamma(A)$, so that by Corollary $1, i_{X}\left(F_{0}, G\right)=i_{X}\left(F_{1}, G\right)$.

Proof. - Assume $A \subset \operatorname{cl}(G)$ and $\gamma(A)>0$. Our first claim is that $g(t)=$ $=\gamma\left(F_{\iota}(A)\right)$ is a continuous function. To see this, select $t_{0} \in J$ and take $\varepsilon>0$. By the uniform continuity of $F_{t}$, select $\delta>0$ such that $\left|t-t_{0}\right|<\delta$ implies $\left\|F_{t}(x)-F_{t_{0}}(x)\right\|<\varepsilon / 2$ for all $x \in A$. Therefore, for $\left|t-t_{0}\right|<\delta, F_{t}(A) \subset N_{\varepsilon / 2}\left(F_{t_{0}}(A)\right)$ and $F_{t_{0}}(A) \subset N_{\varepsilon / 2}\left(F_{t}(A)\right)$. The first inclusion implies $g(t) \leq g\left(t_{0}\right)+\varepsilon$ and the second that $g\left(t_{0}\right)-\varepsilon \leq g(t)$. Thus $h(s)=g(s) / \gamma(A)$ is continuous; and since $h(s)<1$ for $s \in[0,1], h(s) \leq k \leq 1$ for $s \in[0,1]$. It now follows just as in the proof of Proposition 1, Section $E$, that $\gamma(F(A \times J)) \leq k \gamma(A)<\gamma(A)$.

$$
\text { Q.E.D. }
$$

We shall have no need for further generalizations of commutativity and normalization properties of Section $E$, and we shall consequently limit ourselves to the results already stated. We should mention that at least the commutativity property of $E$ easily generalizes to the context of this section.

Remark. - After this paper was written A. VIGNoLI kindly brought to our attention a number of papers in this area which he has written in col. laboration with M. FurI. In particular it seems that the notion of condensing maps was first introduced by Funr and VIGNoLI in [38] under the name "densifying maps». SADovskir cannot be given credit since (as we have already noted) he used a different measure of noncompactness from the one used here. 


\section{REFERENCES}

[1] P. Arexandroff and H. Hopf, Topologie, Berlin, 1935.

[2] A. AmbrosetTi, Un teorema di esistenza per le equazioni differenziali sugli spaai di Banach, Rend. Sem. Mat. Univ. Padua, 39 (1967), 349-361.

[3] JU. G. Borisovic and JU. T. SApronov, A contribution to the topological theory of condensing operators, Soviet Matl. Dokladi, 9 (1968), 1304-1307.

[4] L. Brouwer, Uber abbildungen vom mannigfaltigkeiton, Math. Annalen., 71 (1912), 97.115.

[5] F. E. BRowDER, The topological fixed point theory and its applications to functional analysis, Unpublished Ph.D. Dissertation, Princeton University, 1948.

[6] - -, On the fixed point index for continuons mappings of locally connected spaces, Summa Brasil. Math., 4 (1960), 253-293.

[7] - -, On the spectral theory of elliptic differential operators, Math. Annalen., 142 (1961), 22.130.

[8] - -, Asymptotic fixed point theorems, Math. Annalen., 185 (1970), 38-61.

[9] - -, Nonlinear operators and nonlinear equations of evolution in Banach spaces, Proceedings of the Symposium on Nonlinear Functional Analysis, Amer. Math. Soc., Chicago, April, 1968 (to appear).

[10] - - and R.D. Nussbaun, The topological degree for noncompact nonlinear mappings in Banach spaces, Bull. Amer. Math., Soc., 74 (1968), 641-646.

[11] - - and W. V. PeTRYshyn, Approximation methods and the generalieed topological. degree for nonlinear mappings in Banach spaces, Jour. of Functional Analysis, 3 (1969), 217.24ว.

[12] G. DARBo, Punti uniti in trasformazioni a codominio non compatto, Rond. Sem. Mat. Univ. Padua, 24 (1955), 84.92.

[13] A. Demand, Theorie des points fixes sur les retractes de voisinage des espaces conve. xoides, Bull. Soc. Math. France, 89 (1959), 235.243.

[14] A. DoLD, Fixed point index and fixed point theorems for Euclidean neighborhood retracts, Topology, 4 (1965), 1.8.

[15] J. DugundJ, Ane extension of Tietze's theorem, Pac. Jour. Math., 1 (1951), 353.367.

[16] D.G. de Figureido and L.A. KarLovitz, On the radial projection in normed spaccs, Bull. Amer. Math. Soc., 73 (1936), 364-367.

[17] A. GRANas, Introduction to Topology of Functional Spaces, University of Chicago Mathematics Lecture Notes, Spring, $196^{\circ 1 .}$

[18] O. HANNER, Some theorems on absolute neighborhood retracts, Arkiv for Matomatik, 1 (1951), 389.408.

[19] S. Hu, Theory of Retracts, Detroit (Wayne State University Press), 1965.

[20] C. Kuratowskr, Sur les espaces complets, Fund. Math., 15 (1980), 301.309.

[21] J. LeRAY, Sur la position d'un ensemble ferme de points d'un espace topologique, Jour. de Math. Pures et Appl., 24 (1945), 169-199.

[22] - -, Sur les equations et les transformations, Jour. de Math. Pures et Appl., 24 (1945), 201-248.

[23] - -, Theorie des points fixed, indice total, et nombre de Lefschete, Bull. Soc. Math. France, 87 (1959), 221.283. 
[24] J. Leray and J. Somauder, Topologie et equations fonctionelles, Ann. Sei. Ec. Norm. Sup., $51(1934), 45 \cdot 78$.

[25] M. NAGUMo, A theory of degree of mapping based on infinitesimal analysis, Amer. Jour. Math., 73 (1951), 485.496.

[26] - - Degree of mapping in convex linear topological spaces, Amor. Jour. Math., 73 (1951), 497-511.

[27] M.Z. NASHED and J.S. WONG, Some variants of a fixed point theorem of Krasnoselskii and applications to nonlinear integral equations, Jour. of Math. and Mech., 18 (1969), 767.777 .

[28] R. D. Nussbayx, The fixed point index and asymptotic fixed point theorems for $k$-setcontractions, Bull. Amer. Math. Soc, 75 (1969), 490.495.

[29] - -, The radius of the essential spectrum, Duke Math. Jour., 38 (1970), pp. 473-478.

[30] - - A generalization of the Ascoli theorem and an application to functional differential equations, Jour. Math. Analysis and its Appl., 35 (1971), pp. 600.610.

[31] - --, The fixed point index and fixed point theorems for $k$-set-contractions, Unpublished $\mathrm{Ph} \mathrm{D}$. dissertation (University of Chicago, 1969).

[32] - -, Asymptotic fixed point theorems for local condensing mops, Math. Annalen, 191 (1971), pp. 181-195.

[38] - -, Degree theory for local condensing maps, Jour. Math. Analysis and its Appl. (to appear).

[34] R.S. PaLAIs, Homotopy theory of infinite dimensional manifolds, Topology, 5 (1966), 1.16 .

[35] W.V. PETRYSHYN, On the approximation-solvability of nonlinear equations, Math. Annalen., 177 (1968), 156-164.

[36] B. N. Sadovskr, On a fixed point principle, Functional Analysis and Appl., 1 (1967), 74-76.

[37] R. B. Thompson, A unified approach to local and global fixed point indices, Advances in Math., 3 (1969), 1.72.

[38] M. Furi and A. Vignol, A Fixed point theorem in complete metric spaces, Boll. Unione Matem. Ital, serie IV (N. 4.5), (1969), 505-506.

[39] - - On $\alpha$-nonexpansive mappings and fxed points, Rend. Acc. Naz. Lincei 48 (N. 2), (1970).

[40] - -, On a property of the unit sphere in a linear normed space, Boll. Acad. Pol. Sei., $18(2),(1970), 115 \cdot 116$. 